\title{
Per-phase spatial correlated damage models of UD fibre reinforced composites using mean-field homogenisation; applications to notched laminate failure and yarn failure of plain woven composites
}

\author{
Ling $\mathrm{Wu}^{\mathrm{a}}$, Tianyu Zhang ${ }^{\mathrm{a}}$, Etienne Maillard ${ }^{\mathrm{c}}$, Laurent Adam ${ }^{\mathrm{b}}$, Philippe \\ Martiny ${ }^{\mathrm{b}}$, Ludovic Noels ${ }^{\mathrm{a}}$ \\ ${ }^{a}$ University of Liege, Department of Aeronautics and Mechanical Engineering, \\ Computational \&3 Multiscale Mechanics of Materials, Allée de la découverte 9, B-4000 \\ Liège, Belgium \\ ${ }^{b} M S C$ Software Belgium SA (e-Xstream engineering) \\ Rue Emile Francqui B9, B-1435 Mont-Saint-Guibert Belgium \\ ${ }^{c}$ SONACA S.A., Route Nationale 5, 6041 Gosselies, Belgium
}

\begin{abstract}
A micro-mechanical model for fibre bundle failure is formulated following a phase-field approach and is embedded in a semi-analytical homogenisation scheme. In particular mesh-independence and consistency of energy release rate for fibre bundles embedded in a matrix phase are ensured for fibre dominated failure. Besides, the matrix cracking and fibre-matrix interface debonding are modelled through the evolution of the matrix damage variable framed in an implicit non-local form. Considering the material parameters of both fibre and epoxy matrix phases identified from manufacturer data sheets, it is shown that the failure strength of a ply loaded along the longitudinal
\end{abstract}

Email addresses: 1.wu@ulg.ac.be (Ling Wu), Tianyu.Zhang@uliege.be (Tianyu Zhang), etienne.maillard@sonaca.com (Etienne Maillard), laurent.adam@Hexagon.com (Laurent Adam), philippe.martiny@Hexagon.com (Philippe Martiny), 1.noels@ulg.ac.be (Ludovic Noels) 
direction is in agreement with the reported values. Finally, the multi-damage homogenisation framework is applied to model, on the one hand, the failure of a notched laminate, in which case the failure modes are observed to be in good agreement with experiments, and, on the other hand, the failure of yarns in a plain woven composite unit-cell under uni-axial tension.

Keywords: Mean-field homogenisation, Phase-field, Fibre bundle failure, Matrix cracking, Woven composites, UD laminates

\section{Introduction}

The failure of fibre-reinforced composites often occurs suddenly with3 out any prior visible signs of damage. Understanding and modelling the 4 failure processes of Unidirectional-Carbon Fibre Reinforced Polymer (UD5 CFRP) composite structures become vital to the safe application of compos-

ites. Many attempts had been conducted to predict strength of this kind of material. In recent years, micro in situ experiments and enhanced computer simulations have been carried out to deepen the understanding of failure processes of a UD-CFRP composite component [1-10]. Comparing to homogeneous materials, the failures mechanisms of UD-CFRP composites are more complicated because of the coexistence of fibre-dominated and matrixdominated failure modes and delamination. Delamination of laminated composites has been well modelled with cohesive laws. However, modelling the fibre-dominated and matrix-dominated failure is still an active research area. For matrix-dominated failure, micro-scale modelling was performed with discontinuous Galerkin/cohesive zone method in [6] and with damage enhanced matrix combining cohesive zone at fibre/matrix interface in [4] to 
simulate the transverse tensile failure of UD-CFRP composites. In [5], an XFEM / cohesive zone method was applied to predict the matrix-dominated failure of UD-CFRP composite laminates. The longitudinal tensile failure of UD-CFRP composites is dominated by fibre failure whose mechanisms can be described successively by initiation of single fibre failure accompanied by a redistribution of stress in the neighbouring fibres, the formation and propagation of clusters of broken fibres, and eventually failure of the material. In [7, finite element analyses were applied on representative volume elements (RVEs) with a progressive failure model for fibre bundles. Spring-element model was also used to simulate the failure of fibres in 2D and 3D RVEs [8 10].

It is well understood that the sudden failure is caused by the gradually accumulated micro-damage. Therefore, continuum damage mechanics was also widely used at both macro- and micro-scales in the modelling of composites failures [11 14]. Anisotropic damage models were applied in [11, 12] to describe the degradation of the elastic tensor of a composite ply. The components of the anisotropic damage model were separated into fibre- and matrix-dominated damage processes according to the stress state of the ply. Since the stress state of a ply is a combination of the of stress states in both the matrix and fibre phases, the damage contributions caused by fibre and matrix damage cannot always be clearly separated and this may lead to an inaccurate prediction of the damage zone propagation [15]. Besides, when local damage models are used, the model parameters need to be related to the mesh size of the finite element discretisation in order to reduce the mesh dependency. In a micro-scale finite element analysis on RVEs, damage mod- 
els were introduced in fibre and matrix to predict the longitudinal tensile failure of composites [13].

In the recent years, the phase-field approaches have attracted attention for computational modelling of brittle failure. Using diffusive crack zones governed by a scalar auxiliary variable to mimic the crack surface topology in the solid, the phase-field method does not require the implementation of complex crack tracking algorithms whilst recovering the Griffith fracture approach [16]. At the micro-scale, by considering a combination of phase-field with smeared interfaces [17], it is possible to predict the crack interface interaction. Such an approach was used to develop a micro-mechanical model of the fibre-matrix debonding and matrix cracking interaction [18]. At the macro-scale, a phase-field method with two auxiliary variables, respectively for fibre and inter-fibre failures, was developed in [19] to simulate the crack propagation in UD-CFRP composites. In this approach, the applied constitutive law remains at the composite ply scale, facing the same problem as the other macro-scale anisotropic damage models for which the propagation of crack/damage zones cannot always be captured correctly accordingly to the ply orientation. This particular anisotropic nature of a UD ply can be captured by considering a characteristic lengths tensor with preferred directions in the phase-field equation governing the auxiliary variable [20]. Combining this anisotropic form of the phase-field equation with a new definition of the driving energy release rate, which is defined from the different failure mode strain energies and critical energies, allows recovering the correct crack/damage propagation direction in plies [20] and laminates [21]. We also refer to the recent review of phase-field methods applied to composite lami- 
nates [22]. In these macro-scale models, the parameters are identified by considering the response at the ply level and not directly from the constituents. Besides, the progressive failure mechanism of fibre bundles also physically interacts with the fibre-matrix interface debonding and the matrix yielding and cracking [2] during the failure process of composite materials, and this physical process is difficult to be simulated with a purely macro-scale model.

Clearly, predicting the failure of composites with direct finite element analyses on RVE remains computationally costly when all the coupled damage phenomena are considered while macro-scale models are not detailed enough to represent the interplay between these damage mechanisms, motivating the development of multi-scale methods accounting for the micromechanics. Among the micro-mechanics-based methods, the Mean-Field Homogenisation (MFH) approaches provide an efficient framework to predict the macroscopic behaviour of heterogeneous materials at a reasonable computational cost even for non-linear simulations. Based on the concept of Linear Comparison Composite (LCC) [23, 24], MFH has been extended to the modelling of composites, whose constituents may exhibit non-linear behaviours, as plasticity [25-27] or elasto-visco-plasticity [28 31]. MFH has been extended to consider the damage in the matrix phase independently of the fibre failure in [14]. This method is free from the mesh dependency since the implicit gradient enhanced damage model was adopted [32]. Besides, because of the underlying micro-mechanics model, the matrix damage modes were found to be in good agreement with micro-CT measurements [14]: stress and strain states in fibre and matrix can be estimated in an average sense and the damage in the matrix propagates along the fibre directions even 
for longitudinally loaded plies, as observed in the micro-CT measurements. However, the fibre-dominated failure was not considered in [14].

The fibre strength is a stochastic property that exhibits a size effect [33]. Based on a Weibull distribution of the fibre strength, a stochastic damage model of fibre bundles has been developed and introduced in a Mean Field Homogenisation (MFH) process to describe the fibre breaking in UD fibre reinforced composites [34]. In this model, a length parameter of the stochastic fibre damage model was determined from the matrix and fibre mechanical properties and fibre radius according to the experimental measurements provided in [1], in which optical microscopy was used for in situ measurements of the stress build-up profile of broken fibres. Although fibre failure and matrix cracking were predicted to occur at locations in good agreement with experimental measurements for the longitudinal tensile strength of UD-CFRP notched laminates, the stochastic damage evolution was framed in a local way. As a result fibre damage model needed to be connected with the finite element size and the energy dissipation resulting from fibre-dominated failure could not be resolved.

Embedding damage evolution in a $\mathrm{MFH}$ was shown in [14 to present several advantages resulting from the micro-structure informed nature of the formulation: i) only micro-structure parameters such as the phase material responses have to be identified; ii) the macro-scale resolution also gives information on the phases responses; iii) the anisotropic non-local formulation allowed predicting matrix cracking in good agreement with experimental observations. Nevertheless the method developed in [14] embeds the matrix damage only and is not able to predict laminate failure because of the lack 
of representation of the fibre-dominated failure. The novelty of this work is thus to enrich the non-local matrix damage enhanced MFH formulation to account for fibre failure in a mesh-independent way. Besides because the critical energy release rate of longitudinal failure strongly affects the composite material response, this enrichment ought to be achieved in an energy consistent manner. To address these two requirements, the stochastic fibre damage model developed in [34] and embedded in a Mean Field Homogenisation $(\mathrm{MFH})$ process is substituted by a spatially correlated damage model. In this deterministic approach, it is assumed that the failure results from a stress concentration, in which case the statistical effects become less important than for a uni-axial tension of a uniform sample, and a deterministic continuum damage approach can be a suitable choice. In order to recover mesh-independence and the correct energy release rate for fibre dominated failure, a phase-field model is adopted to describe the embedded fibre bundle failure, allowing recovering the observed physical phenomena in [1]. Furthermore, in this MFH based damage modelling, the behaviours of the fibre and matrix phases are implicitly coupled, which makes the model able to reflect the fibre-matrix interface debonding and the matrix yielding and cracking during fibre breaking via the evolution of the matrix damage variable [34]. This approach is in agreement with the physics observed in composites with strong fibre-matrix interface, in which case, the dominating failure mechanism is an inter-phase failure [35], and the failure of matrix and of interfaces can be both taken into account using a damage-enhanced constitutive model for the matrix [36].

The paper is organised as follows. Section 2 develops the phase-field 
damage model of embedded fibre bundles and the non-local damage model used for the matrix phase. Section 3 details the extension of Mean-Field Homogenisation to account for both matrix cracking and fibre failure. The finite element implementation of the resulting multi-scale framework is formulated in Section 4. The identification of the phases material parameters and the study of the effect of the characteristic failure length of the embedded fibre bundles are provided in Section 5, allowing their determination from experimental measurements. A simple ply tension is then considered in order to evaluate the predicted ply strength. The developed multi-scale model is eventually applied in Section 6 successively to study the failure of a notched laminate and the failure of a plain woven composite unit-cell. The former case was studied with a local approach of fibre bundle damage in 34, in which the simulation exhibited a lack of convergence due to the local damage assumption. In this paper we show that the phase-field approach, on the one hand, allows conducting the simulation to an end, and, on the other hand, predicts the failure modes in good agreement with the experimental CT observations reported in the literature [3. In the latter case studying the failure of a plain woven composite unit-cell, the warps and wefts are modelled as dense unidirectional fibre reinforced epoxy using the developed damage enhanced MFH model, whilst the epoxy matrix out of the yarns is modelled using a non-local damage enhanced elasto-plastic material. The predicted strength of the woven unit-cell is found to be comparable to the experimental observations. 


\section{Mesh-independent damage model of the composite micro-constituents}

In this first section we present the damage enhanced micro-scale constitutive models of the phases of Unidirectional (UD) composite materials. In each phase $\omega_{i}$, at configuration time $t$, the stress tensor can be obtained from a constitutive relation

$$
\boldsymbol{\sigma}(\boldsymbol{x}, t)=\mathcal{S}_{i}\left(\varepsilon\left(\boldsymbol{x}, t_{n+1}\right), \tilde{\boldsymbol{Z}}_{i}(\boldsymbol{x}, \tau) ; \boldsymbol{Z}_{i}(\boldsymbol{x}, \tau), \tau \in[0, t]\right)
$$

where $\boldsymbol{Z}_{i}$ is a set of internal variables particularised to phase $\omega_{i}$ and used to account for history-dependent behaviours. In order to avoid mesh-dependency issues upon strain softening onset, a subset of the internal variables $\boldsymbol{Z}_{i}$ is associated to a set of auxiliary internal variables $\tilde{Z}_{i}$ which are kinematics variables obtained from the resolution of equations that can be stated, for $\tilde{Z}_{i_{j}}$ the internal variable $j$ of phase $\omega_{i}$, in the form

$$
\tilde{Z}_{i_{j}}(\boldsymbol{x}, t)-\boldsymbol{\nabla} \cdot \mathbf{c}_{i} \cdot \boldsymbol{\nabla} \tilde{Z}_{i_{j}}(\boldsymbol{x}, t)=f_{i}\left(Z_{i_{j}}(\boldsymbol{x}, t), \boldsymbol{\varepsilon}(\boldsymbol{x}, t), \tilde{Z}_{i_{j}}(\boldsymbol{x}, t)\right),
$$

where $\mathbf{c}_{i}$ is a squared characteristic lengths matrix associated to phase $\omega_{i}$

and where $f_{i}\left(Z_{i_{j}}, \varepsilon, \tilde{Z}_{i_{j}}\right)$ is a function of the local variable $Z_{i_{j}}$ that depends on the formulation. The constitutive law (1) is then completed by a damage evolution law formulated in a mesh-independent setting, i.e. formulated in terms of the auxiliary internal variables $\tilde{\boldsymbol{Z}}_{i}$, with for phase $\omega_{i}$ :

$$
\left\{\begin{array}{l}
\dot{D}_{i}(\boldsymbol{x}, t)=\mathcal{D}_{i}\left(D_{i}(\boldsymbol{x}, t), \boldsymbol{\varepsilon}(\boldsymbol{x}, t), \boldsymbol{\chi}_{i}(\boldsymbol{x}, t) ; \boldsymbol{Z}_{i}(\boldsymbol{x}, \tau), \tau \in[0, t]\right) \dot{\boldsymbol{\chi}}_{i}, \\
\boldsymbol{\chi}_{i}(\boldsymbol{x}, t)=\max _{\tau \in[0, t]}\left(\tilde{\boldsymbol{Z}}_{i}\right)
\end{array}\right.
$$

where $\chi_{i}$ is the maximum value reached by the auxiliary internal variable in order to ensure irreversibility of the damage process. 
In the following we particularise these equations for the two phases while accounting for the interaction mechanisms between them. First, the case of the failure of fibre bundles embedded in a matrix is studied and framed in a phase-field like approach in order to represent the spatial distribution of the stress build-up developing along a broken embedded fibre. Then, the microcracking of the matrix is developed by combining a non-local approach with an anisotropic squared characteristic lengths matrix in order to account for the presence of fibre bundles which constrain the damage spatial evolution.

\subsection{Phase-field damage model of the embedded fibre bundles}

In this part we introduce a mesh-independent damage model for fibre bundles embedded in a matrix. First the stress build-up resulting from the failure of a single fibre embedded in a matrix is studied. The resulting spatial damage distribution of a fibre bundle is then expressed in terms of an auxiliary damage function defined from a characteristic length, allowing the derivation of phase-field-like governing equations.

\subsubsection{Damage of a broken embedded fibre in a matrix}

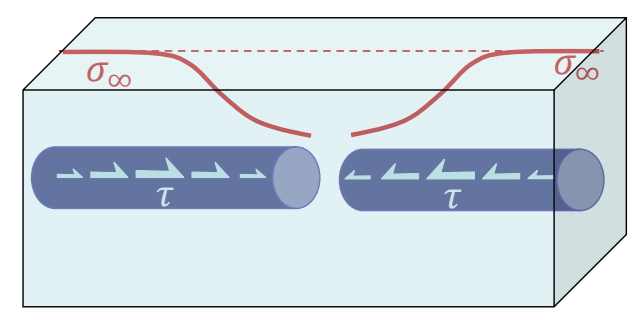

Figure 1: The longitudinal stress build-up at the adjacent parts of the fibre breaking point.

When a fibre embedded in a matrix breaks, the longitudinal stress of this fibre drops to zero at its breaking point whilst the longitudinal stress 
of this fibre increases progressively at the adjacent two sides of the breaking point until the far-field stress $\sigma_{\infty}$ is recovered, see Fig. 1. This progressive longitudinal stress increase in a broken fibre can be described by a stress build-up profile, which is a spatial function of the distance along the fibre with its origin at the breaking point. On the one hand, when embedded in an elastic matrix, the stress build-up profile of a broken fibre can be obtained analytically using the shear-lag theory [37]. On the other hand, when embedded in an elasto-plastic matrix, since the shear stress at fibrematrix interface is limited, either an experimental or a numerical method is required to obtain the stress build-up profile of the broken fibre. Based on the experimental data provided in [1], a continuous function was suggested in [34] to describe the stress profile, which reads

$$
\sigma(x)=\sigma_{\infty}\left(1-\exp \left(-\frac{|x|}{l_{\mathrm{I}}}\right)\right)^{n}
$$

where $|x|$ is the distance from the origin of the fibre breaking point to the considered material point in the longitudinal fibre direction, the length parameter $l_{\mathrm{I}}$ relates to the distance at which the maximum shear stress $\tau$ is reached at the fibre-matrix interface, see Fig. 1, and $n$ is the shape parameter. Values of $n \in[2,3]$ were shown to describe the stress profile $\sigma(x)$ in a good agreement with the experimental data [34].

Since the breaking of an embedded fibre reduces its stress carrying capability from the faraway field to the breaking point, a fibre damage evolution can be defined to describe this decrease in the composite material, which yields

$$
D(x)=1-\frac{\sigma(x)}{\sigma_{\infty}}=1-\left(1-\exp \left(-\frac{|x|}{l_{\mathrm{I}}}\right)\right)^{n} .
$$


Instead of being a local variable, the fibre damage in Eq. (5) is a spatial function characterised by a length parameter $l_{\mathrm{I}}$, with $D(0)=1$ at the fibre breaking point and $D(x) \approx 0.0$ for $|x|>>l_{\mathrm{I}}$. This definition of the fibre damage shows that the effect of the fibre breaking exists in a certain spatial region along the fibre whose size is related to this characteristic length parameter $l_{\mathrm{I}}$.

\subsubsection{Damage of fibre bundle in matrix}

Although a fibre bundle is an aggregate of parallel fibres, its damage evolution cannot be described by a simple linear combination of the damage variables of the individual fibres since the longitudinal stress of a broken fibre will be redistributed to their unbroken neighbours through the matrix. Therefore, the reduction of stress carrying capability of a fibre bundle is also governed by the matrix shear response. However, the concept of effective damage zone with characteristic length $l_{\mathrm{I}}$ introduced when considering a fibre breaking still holds.

As an extreme case, when considering a fibre bundle made of a single fibre, the damage at $x=0$ jumps from 0 at the onset of fibre breaking to 1. When considering several fibres, it is assumed that the damage of the fibre bundle evolves progressively from 0 to 1 at $x=0$ with the increase of longitudinal loading, and, at the ultimate stage $t_{\mathrm{u}}$ of total fibre breaking, one has

$$
D_{\mathrm{I}}\left(x, t_{\mathrm{u}}\right)=1-\left(1-\exp \left(-\frac{|x|}{l_{\mathrm{I}}}\right)\right)^{n},
$$

where, with a view to the upcoming homogenisation process, the subscript "I" of $D_{\mathrm{I}}(x, t)$ refers to the inclusion phase, here the fibre bundle, of the composite material. In order to model a continuous evolution of $D_{\mathrm{I}}(x, t)$ 
in space and during the loading process $t$, an auxiliary function $d_{\mathrm{I}}(x, t)$ is adopted such that

$$
D_{\mathrm{I}}(x, t)=1-\left(1-d_{\mathrm{I}}(x, t)\right)^{n}
$$

with

$$
d_{\mathrm{I}}\left(x, t_{\mathrm{u}}\right)=\exp \left(-\frac{|x|}{l_{\mathrm{I}}}\right),
$$

being the solution of $D_{\mathrm{I}}(x, t)$ at the final breaking stage $t_{\mathrm{u}}$ of the fibre bundle.

It needs to be clarified that $D_{\mathrm{I}}(x, t)$ is a scalar damage variable which mainly describes the degradation of the material along the fibre longitudinal direction represented by the spacial variable $x$. In order to solve the evolution of $D_{\mathrm{I}}(x, t)$ via its auxiliary function $d_{\mathrm{I}}(x, t)$ with a finite-element-based numerical process, a phase-field approach is adopted in this work, which substitutes to Eq. (2), with $D_{\mathrm{I}}(x, t)$ playing the role of the local internal variable $Z_{\mathrm{I}}$ and $d_{\mathrm{I}}(x, t)$ the role of the auxiliary internal variable $\tilde{Z}_{\mathrm{I}}$. Finally, Eq. (7) is the particularised form of the damage evolution law (3).

\subsubsection{Phase-field model}

Phase-field-type approaches use diffusive crack zones governed by a scalar auxiliary variable to mimic the crack surface topology in solid mechanics. The scalar auxiliary variable serves as a measure of the damage, micro-cracks and micro-voids, in a homogenised sense, and its evolution is governed by an evaluation of the related energy dissipation through a new governing equation. In particular, in the work of Miehe [16], to represent a crack surface at $x=0$, the one-dimensional non-smooth phase-field is approximated by an exponential function (8), which is also the sought solution of the fibre bundle damage in Eq. (7) at the ultimate breaking stage $t_{\mathrm{u}}$. Compared to the approach of 
phase-field, in which the auxiliary damage function $d_{\mathrm{I}}\left(x, t_{\mathrm{u}}\right)$ is used to mimic the discontinuous crack surface, in this work $d_{\mathrm{I}}(x, t)$ is used as a measure of the damage evolution in the fibre bundle. Let us note that the fibre damage $D_{\mathrm{I}}$ is defined by Eq. (7) through $d_{\mathrm{I}}$ by

$$
1-D_{\mathrm{I}}=\left(1-d_{\mathrm{I}}\right)^{n} \quad \text { with } \quad n \in[2,3],
$$

and $\left(1-D_{\mathrm{I}}\right)$ is comparable to the stored energy degradation function $g\left(d_{\mathrm{I}}\right)$ defined in [16], which needs to satisfy

$$
g(0)=1, \quad g(1)=0 \quad \text { and } \quad g^{\prime}(1)=0
$$

Energy dissipation of the fibre bundle damaging process. It is assumed that damage is the only energy dissipation mechanism of the fibre bundle and that the energy dissipation can be evaluated through a damage density function following the crack density function as in the work of Miehe [16], with

$$
\gamma\left(d_{\mathrm{I}}, \nabla d_{\mathrm{I}}\right)=\frac{1}{2 l_{\mathrm{I}}} d_{\mathrm{I}}^{2}+\frac{l_{\mathrm{I}}}{2} \nabla d_{\mathrm{I}} \cdot \nabla d_{\mathrm{I}}
$$

The global energy dissipation per unit time related to the damage evolution on an arbitrary volume $\tilde{\omega}$ of the fibre bundle reads

$$
\Phi\left(\dot{d}_{\mathrm{I}} ; d_{\mathrm{I}}\right)=\int_{\tilde{\omega}} \phi\left(\dot{d}_{\mathrm{I}}, \nabla \dot{d}_{\mathrm{I}} ; d_{\mathrm{I}}, \nabla d_{\mathrm{I}}\right) \mathrm{d} V
$$

with the per unit volume and time dissipated energy due to the damage evolution reading

$$
\phi\left(\dot{d}_{\mathrm{I}}, \nabla \dot{d}_{\mathrm{I}} ; d_{\mathrm{I}}, \nabla d_{\mathrm{I}}\right)=G_{c} \dot{\gamma}\left(\dot{d}_{\mathrm{I}}, \nabla \dot{d}_{\mathrm{I}} ; d_{\mathrm{I}}, \nabla d_{\mathrm{I}}\right)+\varepsilon\left\langle\dot{d}_{\mathrm{I}}\right\rangle_{-}^{2},
$$

where $G_{c}$ denotes the dissipated energy at total breaking, i.e. when $D_{\mathrm{I}}\left(0, t_{\mathrm{u}}\right)=$ 1 in Eq. (7), of a fibre bundle of unit cross-section area; this energy corresponds to the critical energy release rate in fracture analysis. In Eq. (13), 
the operator $\varepsilon\langle x\rangle_{-}^{2}$ is the approximated indicator function of the set $\mathbb{R}^{+}$of positive real numbers, with

$$
\langle x\rangle_{-}=(|x|-x) / 2
$$

and the constant $\varepsilon>>1$ being a regularisation parameter of high value, the approximation being exact for the limit $\varepsilon \rightarrow \infty$. This indicator function is introduced in order to ensure the positive evolution of the auxiliary damage variable $\dot{d}_{\mathrm{I}}>0$. As a result, in Eq. (3) one can directly consider $\chi_{\mathrm{I}}=d_{\mathrm{I}}{ }^{1}$

Elastic energy of the fibre bundle. A fibre is modelled using a transverse isotropic linear elastic constitutive law characterised by the elasticity tensor $\mathbb{C}_{\mathrm{I}}^{\mathrm{el}}$. The energy storage function $\psi_{\mathrm{I}}$ describes the strain energy of the fibre stored per unit volume. The energy storage function of an undamaged fibre bundle reads

$$
\psi_{\mathrm{I}}(\varepsilon)=\frac{1}{2} \varepsilon: \mathbb{C}_{\mathrm{I}}^{\mathrm{el}}: \varepsilon,
$$

for a strain tensor $\varepsilon$. The elasticity tensor $\mathbb{C}_{I}^{\mathrm{el}}$ of a transverse isotropic material can be defined by 5 independent elastic constants: the Young's modulus and Poisson's ratio in the 1-2 symmetry plane (transverse plane), $E_{\mathrm{I}}^{1}, \nu_{\mathrm{I}}^{12}$, the Young's modulus and Poisson's ratio in the 3-direction (longitudinal direction), $E_{\mathrm{I}}^{3}, \nu_{\mathrm{I}}^{31}$ and the shear modulus in the 3 -direction, $\mu_{\mathrm{I}}^{31}$. The other parameters can be derived through some relations, such as $E_{\mathrm{I}}^{1}=E_{\mathrm{I}}^{2}$ and $\frac{\nu_{\mathrm{I}}^{13}}{E_{\mathrm{I}}^{1}}=\frac{\nu_{\mathrm{I}}^{31}}{E_{\mathrm{I}}^{3}}$. The longitudinal direction of the fibres is refereed to by the superscript 3, and its two symmetric transverse directions by the superscript 1

\footnotetext{
${ }^{1}$ During the implementation we however keep the formulation (3) instead of considering the term $\varepsilon\langle x\rangle_{-}^{2}$ because convergence was shown to be better.
} 
where $\Delta=\left(1+\nu_{\mathrm{I}}^{12}\right)\left(1-\nu_{\mathrm{I}}^{12}-2 \nu_{\mathrm{I}}^{13} \nu_{\mathrm{I}}^{31}\right)$.

It is here assumed that the fibre bundle damage is only due to a tension along the longitudinal fibre direction. However the damage affects the energy storage of fibre in both longitudinal tension and compression modes because of the resulting material degradation. Since the breaking of a fibre can cause a local debonding and/or bonding degradation at the fibre matrix interface, this assumption is reasonable. Therefore, the energy storage function of a damaged fibre bundle reads

$$
\psi_{\mathrm{I}}\left(\varepsilon, d_{\mathrm{I}}\right)=\psi_{\mathrm{I}}^{+}\left(\varepsilon, d_{\mathrm{I}}\right)+\psi_{\mathrm{I}}^{-}\left(\varepsilon ; d_{\mathrm{I}}\right),
$$

where the positive part $\psi_{\mathrm{I}}^{+}\left(\varepsilon, d_{\mathrm{I}}\right)$ refers to fibres in tension and the negative part $\psi_{\mathrm{I}}^{-}\left(\varepsilon ; d_{\mathrm{I}}\right)$, in which $d_{\mathrm{I}}$ is seen as a constant parameter and no longer as an evolving variable, refers to the fibre in compression. Defining $\mathbb{C}_{I}^{D}$ as the damaged elasticity tensor defined through the damage variable $D_{\text {I }}$ given by 
Eq. (7), one has

$$
\psi_{\mathrm{I}}\left(\varepsilon, d_{\mathrm{I}}\right)=\frac{1}{2} \varepsilon: \mathbb{C}_{\mathrm{I}}^{\mathrm{D}}: \boldsymbol{\varepsilon} .
$$

$$
\begin{aligned}
E_{\mathrm{I}}^{3 \mathrm{D}} & =\left(1-D_{\mathrm{I}}\right) E_{\mathrm{I}}^{3}, \text { and } \\
\nu_{\mathrm{I}}^{31 \mathrm{D}} & =\left(1-D_{\mathrm{I}}\right) \nu_{\mathrm{I}}^{31},
\end{aligned}
$$

where the second equation allows keeping $\frac{\nu_{1}^{13}}{E_{1}^{1}}$ constant and $\frac{\nu_{1}^{31}}{E_{1}^{3}}=\frac{\nu_{1}^{31} \mathrm{D}}{E_{1}^{3 \mathrm{D}}}$, yielding a damaged transverse isotropic elasticity tensor, which reads using Voigt's notations:

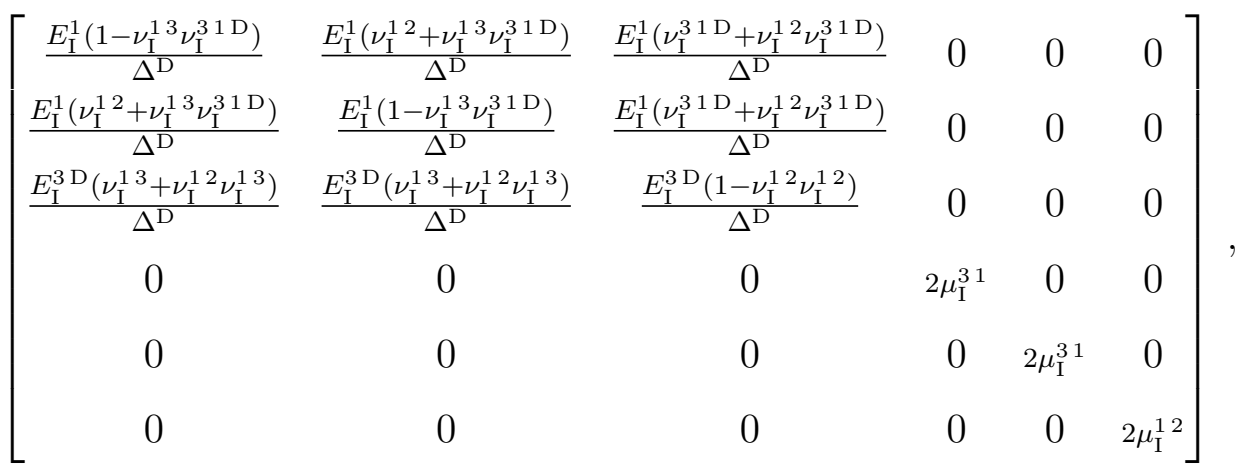

where $\Delta^{\mathrm{D}}=\left(1+\nu_{\mathrm{I}}^{12}\right)\left(1-\nu_{\mathrm{I}}^{12}-2 \nu_{\mathrm{I}}^{13} \nu_{\mathrm{I}}^{31 \mathrm{D}}\right)$. 
The global bulk energy storage on an arbitrary volume $\tilde{\omega}$ of the fibre bundle reads

$$
\Psi_{\mathrm{I}}\left(\varepsilon, d_{\mathrm{I}}\right)=\int_{\tilde{\omega}} \psi_{\mathrm{I}}\left(\varepsilon, d_{\mathrm{I}}\right) \mathrm{d} V,
$$

and according to the assumption (17), the evolution of the stored energy reads

$$
\dot{\Psi}_{\mathrm{I}}\left(\dot{\varepsilon}, \dot{d}_{\mathrm{I}} ; \varepsilon, d_{\mathrm{I}}\right)=\int_{\tilde{\omega}} \dot{\psi}_{\mathrm{I}} \mathrm{d} V=\int_{\tilde{\omega}}\left[\frac{\partial \psi_{\mathrm{I}}}{\partial \varepsilon} \dot{\boldsymbol{\varepsilon}}+\frac{\partial \psi_{\mathrm{I}}^{+}}{\partial d_{\mathrm{I}}} \dot{d}_{\mathrm{I}}\right] \mathrm{d} V .
$$

The classical constitutive assumption yields the stress expression (1) of the fibre bundle which, using Eq. (18), reads

$$
\boldsymbol{\sigma}=\frac{\partial \psi_{\mathrm{I}}}{\partial \boldsymbol{\varepsilon}}=\mathbb{C}_{\mathrm{I}}^{\mathrm{D}}: \boldsymbol{\varepsilon}
$$

The governing equation for $d_{I}$. The balance of mechanical energy on the arbitrary volume $\tilde{\omega}$ requires that

$$
\dot{\Psi}_{\mathrm{I}}\left(\dot{\boldsymbol{\varepsilon}}, \dot{d}_{\mathrm{I}} ; \boldsymbol{\varepsilon}, d_{\mathrm{I}}\right)+\Phi\left(\dot{d}_{\mathrm{I}}\right)=\dot{P}(\dot{\boldsymbol{u}}),
$$

where $\dot{P}(\dot{\boldsymbol{u}})$ is the external power, and $\boldsymbol{u}$ is the displacement field. Equation (26) needs to be satisfied for all admissible rates $\dot{\boldsymbol{u}}$ and $\dot{d}_{\mathrm{I}}$. Using the expressions (12) and (23), Eq. (26) is rewritten as

$$
\int_{\tilde{\omega}}\left[\frac{\partial \psi_{\mathrm{I}}}{\partial \boldsymbol{\varepsilon}} \dot{\boldsymbol{\varepsilon}}+\frac{\partial \psi_{\mathrm{I}}^{+}}{\partial d_{\mathrm{I}}} \dot{d}_{\mathrm{I}}+\phi\left(\dot{d}_{\mathrm{I}}, \nabla \dot{d}_{\mathrm{I}} ; d_{\mathrm{I}}, \nabla d_{\mathrm{I}}\right)\right] \mathrm{d} V=\dot{P}(\dot{\boldsymbol{u}}) .
$$


The expression of $\dot{P}(\dot{\boldsymbol{u}})$ is not directly available for the fibres embedded in the matrix, and some micro-mechanics assumptions are required to derive the equations related to the strain rate $\dot{\boldsymbol{\varepsilon}}$ (and displacement rate $\dot{\boldsymbol{u}}$ ) evolution in the composite phases; this point will be studied in Section 3.1 when performing the homogenisation process.

Considering only the admissible damage rate $\dot{d}_{\mathrm{I}}$ in Eq. (27) allows extracting the missing governing law. Using Eqs. (11) and (13) leads to

$$
\int_{\tilde{\omega}}\left[\frac{\partial \psi_{\mathrm{I}}^{+}}{\partial d_{\mathrm{I}}} \dot{d}_{\mathrm{I}}+G_{c}\left(\frac{1}{l_{\mathrm{I}}} d_{\mathrm{I}} \dot{d}_{\mathrm{I}}+l_{\mathrm{I}} \nabla d_{\mathrm{I}} \cdot \nabla \dot{d}_{\mathrm{I}}\right)-\varepsilon\left\langle\dot{d}_{\mathrm{I}}\right\rangle_{-} \dot{d}_{\mathrm{I}}\right] \mathrm{d} V=0 .
$$

The application of the Gauss theorem on the term " $\nabla d_{\mathrm{I}} \cdot \nabla \dot{d}_{\mathrm{I}}$ " of Eq. 28 gives

$$
\begin{array}{r}
\int_{\tilde{\omega}}\left[\frac{\partial \psi_{\mathrm{I}}^{+}}{\partial d_{\mathrm{I}}} \dot{d}_{\mathrm{I}}+G_{c}\left(\frac{1}{l_{\mathrm{I}}} d_{\mathrm{I}}-l_{\mathrm{I}} \nabla^{2} d_{\mathrm{I}}\right) \dot{d}_{\mathrm{I}}-\varepsilon\left\langle\dot{d}_{\mathrm{I}}\right\rangle_{-} \dot{d}_{\mathrm{I}}\right] \mathrm{d} V+ \\
G_{c} l_{\mathrm{I}} \int_{\partial \tilde{\omega}} \nabla d_{\mathrm{I}} \cdot \boldsymbol{n} \dot{d}_{\mathrm{I}} \mathrm{d} S=0,
\end{array}
$$

where $\boldsymbol{n}$ is the outward normal on $\partial \tilde{\omega}$. The governing equation of $d_{\mathrm{I}}$ can then be obtained as

$$
d_{\mathrm{I}}-l_{\mathrm{I}}^{2} \nabla^{2} d_{\mathrm{I}}-\frac{l_{\mathrm{I}}}{G_{c}} \varepsilon\left\langle\dot{d}_{\mathrm{I}}\right\rangle_{-}=-\frac{l_{\mathrm{I}}}{G_{c}} \frac{\partial \psi_{\mathrm{I}}^{+}}{\partial d_{\mathrm{I}}},
$$

which is the particularised form of Eq. (2). The algorithmic operators of $\left(-\frac{l_{\mathrm{I}}}{G_{c}} \frac{\partial \psi_{I}^{+}}{\partial d_{\mathrm{I}}}\right)\left(\varepsilon, d_{\mathrm{I}}\right)$ are given in Appendix A.1.1.

\subsection{Non-local damage model of the matrix phase}

In this part, the damage model of the matrix is framed in an implicit nonlocal form as suggested in [32, 38, 39]. However to account for the fact that the fibre bundles embedded in the matrix govern the direction of the damage propagation, the non-local model uses an anisotropic squared characteristic lengths matrix as suggested in [14]. 


\subsubsection{Non-local damage enhanced $J_{2}$ plasticity}

The constitutive Eq. (1) is particularised to the case of an elasto-plastic material enhanced by a non-local damage model.

Considering that the strain tensors in the actual and undamaged or effective phase representations are equivalent [40], the effective or undamaged stress $\hat{\boldsymbol{\sigma}}(\boldsymbol{x}, t)$ is defined from the apparent stress $\boldsymbol{\sigma}(\boldsymbol{x}, t)$ by introducing a damage parameter $0 \leq D_{0}(\boldsymbol{x}, t)<1$, such that

$$
\hat{\boldsymbol{\sigma}}=\frac{\boldsymbol{\sigma}}{\left(1-D_{0}\right)},
$$

where the subscript " 0 " of $D_{0}(\boldsymbol{x}, t)$ refers to the matrix phase.

In the context of $J_{2}$ elasto-plasticity, and assuming that the plastic flow equations can be written in the effective stress space, the von Mises stress criterion reads

$$
f=\hat{\sigma}^{\mathrm{eq}}-R_{0}\left(p_{0}\right)-\sigma_{Y_{0}} \leqslant 0,
$$

with the equivalent von Mises effective stress $\hat{\sigma}^{\text {eq }}=\sqrt{\frac{3}{2} \frac{\operatorname{dev}(\boldsymbol{\sigma})}{1-D_{0}}: \frac{\operatorname{dev}(\boldsymbol{\sigma})}{1-D_{0}}}$, the yield surface $f$, the initial yield stress $\sigma_{Y_{0}}$, and the isotropic hardening stress $R_{0}\left(p_{0}\right) \geqslant 0$, where $p_{0}$ is the internal variable characterising the irreversible behaviour, here the equivalent plastic strain 2 , The plastic flow rule, see Appendix A.2.1, yields the plastic strain tensor $\varepsilon^{\mathrm{pl}}$. The set of internal variables $\boldsymbol{Z}_{0}$ is thus $\left\{p_{0}, \boldsymbol{\varepsilon}^{\mathrm{pl}}\right\}$.

In a small deformations context, the reversible (elastic) and irreversible (plastic) strain tensors can be added $\left(\varepsilon=\varepsilon^{\mathrm{el}}+\varepsilon^{\mathrm{pl}}\right)$, allowing to particularise

\footnotetext{
${ }^{2}$ Rigorously, the von Mises stress criterion 32 should be written $f(\hat{\boldsymbol{\sigma}}, r) \leqslant 0$, where $r$ is an internal variable related to the accumulated plastic strain $p_{0}$ and to the plastic multiplier $\dot{\lambda}$ following $\dot{r}=\dot{\lambda}=\left(1-D_{0}\right) \dot{p}_{0}$, see the discussion by [41] for details.
} 
275

$$
D_{0}=\frac{D_{\max _{0}}}{1-\frac{1}{1+\exp \left(s_{0} p_{\mathrm{C}_{0}}\right)}}\left(\frac{1}{1+\exp \left(-s_{0}\left(\chi_{0}-p_{\mathrm{C}_{0}}\right)\right)}-\frac{1}{1+\exp \left(s_{0} p_{\mathrm{C}_{0}}\right)}\right)
$$

with the fourth-order Hooke tensor of the undamaged elasticity tensor of the matrix reading

$$
\mathbb{C}_{0}^{\mathrm{el}}=3 \kappa_{0} \mathbb{I}^{\mathrm{vol}}+2 \mu_{0} \mathbb{I}^{\mathrm{dev}} .
$$

In this last equation, $\kappa_{0}$ and $\mu_{0}$ are the elastic bulk and shear modulii of the undamaged material and $\mathbb{I}^{\mathrm{vol}}=\frac{1}{3} \boldsymbol{I} \otimes \boldsymbol{I}$ and $\mathbb{I}^{\mathrm{dev}}=\mathbb{I}-\mathbb{I}^{\mathrm{vol}}$ are respectively the spherical and deviatoric operators.

\subsubsection{Damage evolution laws}

The damage evolution law (3) is formulated in a non-local setting with as a set of non-local internal variables $\tilde{\boldsymbol{Z}}_{0}$, the scalar $\left\{\tilde{p}_{0}\right\}$, which is the non-local counterpart of the equivalent plastic strain $p_{0} \in \boldsymbol{Z}_{0}$.

One possible damage evolution law is the classical Lemaitre-Chaboche law [42]

$$
\dot{D}_{0}=\left(\frac{\psi_{0}(\varepsilon)}{S_{0}}\right)^{s_{0}} \dot{\chi}_{0} \quad \text { when } \quad\left(\chi_{0}-p_{\mathrm{C}_{0}}\right) \dot{\chi}_{0}>0
$$

where $S_{0}, s_{0}$ and the damage critical plastic strain $p_{\mathrm{C}_{0}}$ are the material parameters, and $\psi_{0}(\varepsilon)$ is the strain energy release rate computed as

$$
\psi_{0}(\varepsilon)=\frac{1}{2} \varepsilon^{\mathrm{el}}: \mathbb{C}_{0}^{\mathrm{el}}: \varepsilon^{\mathrm{el}} .
$$

Another possible damage law is to saturate the damage evolution with 
where $D_{\max _{0}}$ is the saturation damage and $s_{0}, p_{\mathrm{C}_{0}}$ are two material parameters.

In these equations, $\chi_{0}(\boldsymbol{x}, t)=\max _{\tau \in[0, t]}\left(\tilde{p}_{0}\right)$ ensures the irreversibility of the damage evolution.

The algorithmic operators of $\boldsymbol{\sigma}\left(\boldsymbol{\varepsilon}, \tilde{p}_{0}\right)$ are given in Appendix A.2.2.

\subsubsection{Governing equation for $\tilde{p}_{0}$}

The damage evolution law (3) was particularised in the previous section with as non-local internal variables $\tilde{\boldsymbol{Z}}_{0}$, the scalar $\left\{\tilde{p}_{0}\right\}$, which is evaluated from its local counterpart $p_{0} \in \boldsymbol{Z}_{0}$ using the implicit non-local model [32, 38, 39], which reads

$$
\tilde{p}_{0}-\nabla \cdot\left(\boldsymbol{c}_{0} \cdot \nabla \tilde{p}_{0}\right)=p_{0},
$$

where $\boldsymbol{c}_{0}$ is the matrix of the squared characteristic lengths. Because of the presence of the fibre bundles in the matrix, a longer non-local length along the UD-fibre direction was suggested in [14] in order to represent the interaction with the fibres which "block" the matrix material-point interactions in the transverse directions of UD-fibre, whilst "prolonging" it in the longitudinal direction.

The algorithmic operators of $p_{0}\left(\varepsilon, \tilde{p}_{0}\right)$ are given in Appendix A.2.2.

\section{MFH with damage-enhanced matrix and fibres}

In this section we derive a Mean-Field Homogenisation (MFH) framework accounting for the damage distribution in a Unidirectional (UD) composite material in a non-local way. First, the key principles of Mean-Field Homogenisation $(\mathrm{MFH})$ are recalled in the cases of linear and non-linear 
two-phase composite materials. The incremental-secant MFH method for non-linear composites is then developed in order to account for the damage evolution in both the fibre bundle and matrix phases. In particular, the phase-field like fibre bundle damage model developed in Section 2.1 is used to derive the damage evolution of the inclusion phase, whilst the non-local damage model developed in Section 2.2 is used to derive the damage evolution in the matrix phase as previously done in [14, 43].

\subsection{Mori-Tanaka-based MFH for composites}

Homogenisation theories provide the relation between the macro-strains $\varepsilon_{\mathrm{M}}$ and macro-stresses $\boldsymbol{\sigma}_{\mathrm{M}}$ under the form of a relation between the volume averages of the micro-strains $\varepsilon_{\mathrm{m}}(\boldsymbol{x})$ and micro-stresses $\boldsymbol{\sigma}_{\mathrm{m}}(\boldsymbol{x})$ over a mesoscale volume element $\omega$, with

$$
\varepsilon_{\mathrm{M}}=\left\langle\varepsilon_{\mathrm{m}}(\boldsymbol{x})\right\rangle_{\omega} \quad \text { and } \quad \boldsymbol{\sigma}_{\mathrm{M}}=\left\langle\boldsymbol{\sigma}_{\mathrm{m}}(\boldsymbol{x})\right\rangle_{\omega}
$$

where $\langle f(\boldsymbol{x})\rangle_{\omega}=\frac{1}{V_{\omega}} \int_{\omega} f(\boldsymbol{x}) d V$ and $V_{\omega}$ is the volume of the meso-scale volume element $\omega$.

These relations can be particularised in the context of a two-phase isothermal composite material by separating the volume averages on the matrix subdomain $\omega_{0}$ and on the inclusions subdomain $\omega_{\text {I }}$ following

$$
\varepsilon_{\mathrm{M}}=v_{0}\left\langle\varepsilon_{\mathrm{m}}\right\rangle_{\omega_{0}}+v_{\mathrm{I}}\left\langle\varepsilon_{\mathrm{m}}\right\rangle_{\omega_{\mathrm{I}}} \text { and } \quad \boldsymbol{\sigma}_{\mathrm{M}}=v_{0}\left\langle\boldsymbol{\sigma}_{\mathrm{m}}\right\rangle_{\omega_{0}}+v_{\mathrm{I}}\left\langle\boldsymbol{\sigma}_{\mathrm{m}}\right\rangle_{\omega_{\mathrm{I}}}
$$

where the respective volume fractions $v_{i}$ obey to $v_{0}+v_{\mathrm{I}}=1$. As a convention, the subscript "0" refers to the matrix phase and the subscript "I" to the inclusion phase. In what follows, the notations $\left\langle\bullet_{\mathrm{m}}\right\rangle_{\omega_{i}}$ are replaced by $\langle\bullet\rangle_{i}$ for conciseness. 
First-statistical moment mean-field homogenisation assumes that the composite material response can be evaluated by applying the phases constitutive behaviour on the average strain $\langle\boldsymbol{\varepsilon}\rangle_{i}$ and stress $\langle\boldsymbol{\sigma}\rangle_{i}$ tensors of the phase $\omega_{i}$. However, they require further assumptions under the form of a relation between the average strain $\langle\boldsymbol{\varepsilon}\rangle_{i}$ tensors of the two phases. A commonly used assumption for 2-phase composite materials is the Mori-Tanaka extension of the Eshelby single inclusion solution [44] to multiple-inclusion interactions since it provides accurate predictions [45]. This assumption is first recalled in the linear range and then extended in the non-linear range by defining a Linear Comparison Composite (LCC) material.

\subsubsection{Case of linear elasticity}

Assuming linear elasticity for both phases, considering a linear elastic behaviour that can be applied on the average strain $\langle\boldsymbol{\varepsilon}\rangle_{i}$ and stress $\langle\boldsymbol{\sigma}\rangle_{i}$ tensors of the phase $\omega_{i}$, yields

$$
\langle\boldsymbol{\sigma}\rangle_{0}=\mathbb{C}_{0}^{\mathrm{el}}:\langle\boldsymbol{\varepsilon}\rangle_{0} \quad \text { and } \quad\langle\boldsymbol{\sigma}\rangle_{\mathrm{I}}=\mathbb{C}_{\mathrm{I}}^{\mathrm{el}}:\langle\boldsymbol{\varepsilon}\rangle_{\mathrm{I}},
$$

where $\mathbb{C}_{0}^{\text {el }}$ is the uniform elasticity tensor of the matrix phase and $\mathbb{C}_{\mathrm{I}}^{\text {el }}$ is the uniform elasticity tensor of the inclusion phase.

The relation linking the strain averages per phase can be stated under the form

$$
\langle\varepsilon\rangle_{\mathrm{I}}=\mathbb{B}^{\epsilon}\left(\mathrm{I}, \mathbb{C}_{0}^{\mathrm{el}}, \mathbb{C}_{\mathrm{I}}^{\mathrm{el}}\right):\langle\varepsilon\rangle_{0},
$$

where $\mathbb{B}^{\epsilon}$ is the strain concentration tensor whose expression depends on the chosen micro-mechanics assumptions, on "I", the geometrical information of the inclusion phase, and on the elasticity tensors of both phases. In case of 
the Mori-Tanaka (M-T) [46] assumption, this tensor reads

$$
\mathbb{B}^{\epsilon}\left(\mathrm{I}, \mathbb{C}_{0}, \mathbb{C}_{\mathrm{I}}\right)=\left\{\mathbb{I}+\mathbb{S}\left(\mathrm{I}, \mathbb{C}_{0}\right):\left[\left(\mathbb{C}_{0}\right)^{-1}: \mathbb{C}_{\mathrm{I}}-\mathbb{I}\right]\right\}^{-1},
$$

where $\mathbb{C}_{0}$ and $\mathbb{C}_{\mathrm{I}}$ are the considered phase linear operators, i.e. respectively $\mathbb{C}_{0}^{\mathrm{el}}$ and $\mathbb{C}_{\mathrm{I}}^{\mathrm{el}}$ in the context of linear elasticity, and where the Eshelby tensor $\mathbb{S}\left(\mathrm{I}, \mathbb{C}_{0}\right)$ 44 depends on "I", the geometrical information of the inclusions, and on the linear operator $\mathbb{C}_{0}$ of the matrix phase.

For linear elastic composites, the set of Eqs. 40, 42 can be rewritten as the following macro-scale constitutive relation

$$
\boldsymbol{\sigma}_{\mathrm{M}}=\mathbb{C}_{\mathrm{M}}^{\mathrm{el}}\left(\mathrm{I}, \mathbb{C}_{0}^{\mathrm{el}}, \mathbb{C}_{\mathrm{I}}^{\mathrm{el}}, v_{\mathrm{I}}\right): \boldsymbol{\varepsilon}_{\mathrm{M}}
$$

\subsubsection{Definition of Linear Comparison Composite (LCC)}

$\mathrm{MFH}$ can be extended to the non-linear range by considering an incremental form between the configurations at time $t_{n}$ and at time $t_{n+1}$. To this end, a Linear Comparison Composite (LCC) [23, 24, 27, 47 53] is defined during that time increment as a virtual heterogeneous material, whose constituents linear behaviours, defined through virtual elastic operators, match the linearised behaviours of the real composite material constituents at that configurations. The LCC definition yields virtual elastic operators $\mathbb{C}_{0}^{\text {LCC }}$ of the matrix phase and $\mathbb{C}_{\mathrm{I}}^{\mathrm{LCC}}$ of the inclusion phase, allowing the MFH equations of the linear composite material developed in Section 3.1.1 to be applied readily. In particular, the set of Eqs. 40 is thus rewritten as

$$
\Delta \varepsilon_{\mathrm{M}}=v_{0}\langle\Delta \varepsilon\rangle_{0}+v_{\mathrm{I}}\langle\Delta \varepsilon\rangle_{\mathrm{I}} \quad \text { and } \quad \boldsymbol{\sigma}_{\mathrm{M}}=v_{0}\langle\boldsymbol{\sigma}\rangle_{0}+v_{\mathrm{I}}\langle\boldsymbol{\sigma}\rangle_{\mathrm{I}}
$$

and the relation $(42)$ is rewritten using the averaged incremental strains in the two phases as

$$
\langle\Delta \varepsilon\rangle_{\mathrm{I}}=\mathbb{B}^{\epsilon}\left(\mathrm{I}, \mathbb{C}_{0}^{\mathrm{LCC}}, \mathbb{C}_{\mathrm{I}}^{\mathrm{LCC}}\right):\langle\Delta \varepsilon\rangle_{0}
$$


These equations are completed by the constitutive behaviour models (1) of the phases, but written in terms of the average stress and strain tensors at configuration time $t_{n+1}$ in the phase $\omega_{i}$, yielding

$$
\langle\boldsymbol{\sigma}\rangle_{i}\left(t_{n+1}\right)=\mathcal{S}_{i}\left(\langle\boldsymbol{\varepsilon}\rangle_{i}\left(t_{n+1}\right), \tilde{\boldsymbol{Z}}_{i}(\tau) ; \boldsymbol{Z}_{i}(\tau), \tau \in[0, t]\right)
$$

where $\boldsymbol{Z}_{i}$ and $\tilde{\boldsymbol{Z}}_{i}$, the sets of internal and auxiliary variables used to account for history-dependent behaviours of phase $\omega_{i}$, are considered as uniform on the phase $\omega_{i}$. However, they are not strictly volume average values, which explains why the notation $\langle\bullet\rangle_{i}$ is not used.

\subsection{Incremental-secant MFH with damage model in both phases}

Different assumptions on the linearisation method were made to define the LCC. In [27], a virtual unloading step of the composite material was first applied, and then followed by a secant loading from the residual states reached in both phases. This so-called incremental-secant approach uses the loading step in order to define the virtual elastic operators $\mathbb{C}_{0}^{\mathrm{LCC}}$ and $\mathbb{C}_{\mathrm{I}}^{\mathrm{LCC}}$ of the LCC. The virtual unloading allows improving the accuracy in the case of non-proportional loading [27] and in the case of damage-enhanced elasto-plasticity of the matrix phase since it allows capturing the fibre elastic unloading occurring during the matrix softening [43].

This method is extended in this paper to account for the phase-field formulation of the fibre bundle damage model developed in Section 2.1.

\subsubsection{Virtual elastic unloading}

The virtual elastic unloading is defined as an unloading process of the composite material at configuration $t_{n}$ in order to reach a residual stress 


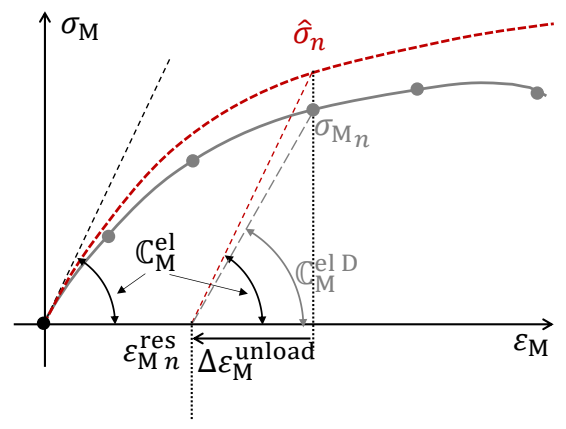

(a) Composite material; unloading

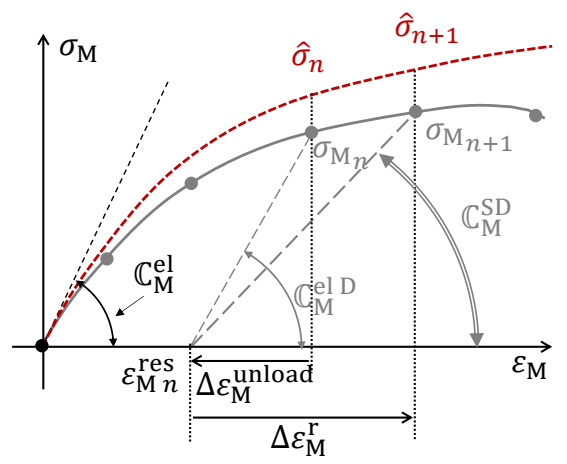

(c) Composite material; loading

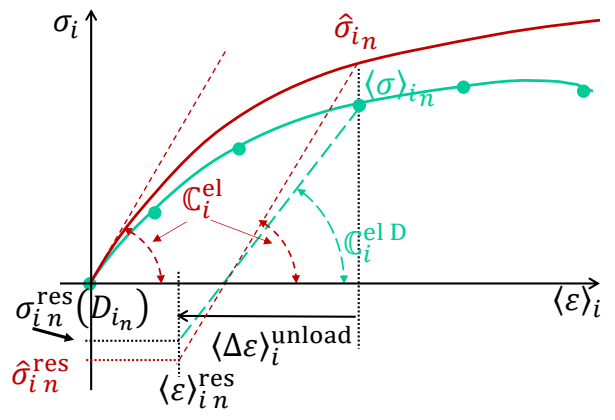

(b) Phase $\omega_{i}$; unloading

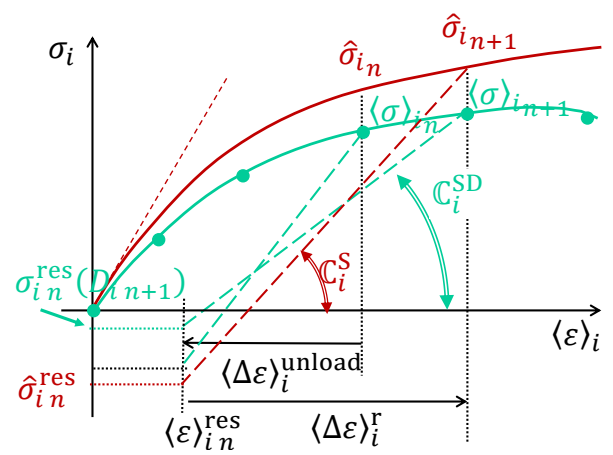

(d) Phase $\omega_{i}$; loading

Figure 2: Definition of the LCC in the incremental-secant method for damage-enhanced elasto-plastic composites: (a) Virtual elastic unloading of the composite material with the elastic operator $\mathbb{C}_{\mathrm{M}}^{\mathrm{el}} \mathrm{D}$, the red dotted line corresponds to an undamaged composite material and is shown for illustration purpose only; (b) Corresponding virtual elastic unloading of an elasto-plastic phase $\omega_{i}$ with the damaged elastic operator $\mathbb{C}_{i}^{\text {el D }}$, the red line corresponds to the effective stress-strain curve (or undamaged phase material); (c) Incremental-secant loading of the composite material from the virtually unloaded state and definition of the incremental-secant operator $\mathbb{C}_{\mathrm{M}}^{\mathrm{SD}}$; and (d) Corresponding incremental-secant loading of a damage-enhanced elasto-plastic phase $\omega_{i}$ from the residual undamaged stress and definition of the incremental-secant phase operator $\mathbb{C}_{i}^{S}$; the damaged incremental-secant phase operator $\mathbb{C}_{i}^{\mathrm{SD}}$ is obtained in the apparent stress space. 
state $\boldsymbol{\sigma}_{\mathrm{M} n}^{\mathrm{res}}=0$, where the superscript "res" refers to the virtually unloaded state. It is assumed that this unloading process does not involve reverse plasticity which can always be stated since the unloading remains virtual. The case of damage-enhanced elasto-plasticity is illustrated in Figs. 2(a) and 2(b) for respectively the composite material and the phase $\omega_{i}$.

Since this virtual unloading is elastic, the LCC is defined from the phase damaged elastic operators, i.e. $\mathbb{C}_{0}^{\mathrm{el} \mathrm{D}}=\left(1-D_{0}\right) \mathbb{C}_{0}^{\mathrm{el}}$ following Eq. (33) for the matrix phase $\omega_{0}$, and $\mathbb{C}_{\mathrm{I}}^{\mathrm{el} D}=\mathbb{C}_{\mathrm{I}}^{\mathrm{D}}\left(D_{\mathrm{I}}\right)$ following Eq. 24 for the fibre bundle phase $\omega_{\mathrm{I}}$. These operators are constant during the virtual unloading step since elasticity is assumed to occur at constant damage variables.

The unloading is obtained from Eq. (44) by setting a macro-stress equal to zero, yielding

$$
0=\boldsymbol{\sigma}_{\mathrm{M} n}-\mathbb{C}_{\mathrm{M}}^{\mathrm{el} \mathrm{D}}\left(\mathrm{I}, \mathbb{C}_{0}^{\mathrm{el} \mathrm{D}}, \mathbb{C}_{\mathrm{I}}^{\mathrm{el} \mathrm{D}}, v_{\mathrm{I}}\right): \Delta \boldsymbol{\varepsilon}_{\mathrm{M}}^{\text {unload }},
$$

with

$$
\begin{aligned}
\mathbb{C}_{\mathrm{M}}^{\mathrm{el}}= & {\left[v_{\mathrm{I}} \mathbb{C}_{\mathrm{I}}^{\mathrm{el} \mathrm{D}}: \mathbb{B}^{\epsilon}\left(\mathrm{I}, \mathbb{C}_{0}^{\mathrm{el} \mathrm{D}}, \mathbb{C}_{\mathrm{I}}^{\mathrm{el} \mathrm{D}}\right)+v_{0} \mathbb{C}_{0}^{\mathrm{el} \mathrm{D}}\right]: } \\
& {\left[v_{\mathrm{I}} \mathbb{B}^{\epsilon}\left(\mathrm{I}, \mathbb{C}_{0}^{\mathrm{el} \mathrm{D}}, \mathbb{C}_{\mathrm{I}}^{\mathrm{el} \mathrm{D}}\right)+v_{0} \mathbb{I}\right]^{-1}, }
\end{aligned}
$$

the macro-scale damaged elastic operator $\mathbb{C}_{\mathrm{M}}^{\mathrm{el} D}$ obtained from the damaged elastic operators $\mathbb{C}_{0}^{\mathrm{el} D}$ and $\mathbb{C}_{\mathrm{I}}^{\mathrm{el} D}$ of both phases, see Fig. 2(b).

The residual states in the phases are deduced from the set of Eqs. 45 . 46. The virtual unloading of the composite material results in residual strain tensors $\langle\varepsilon\rangle_{i_{n}}^{\text {res }}=\langle\varepsilon\rangle_{i_{n}}-\langle\Delta \varepsilon\rangle_{i}^{\text {unload }}$ and residual stress tensors $\langle\boldsymbol{\sigma}\rangle_{i_{n}}^{\text {res }}$ in the two phases as depicted in Fig. 2(b). The apparent residual stress obtained in phase $\omega_{i}$ after unloading at configuration $n$ is denoted by $\boldsymbol{\sigma}_{i_{n}}^{\text {res }}\left(D_{i_{n}}\right)$, because 
see Fig. 2(d).

The linear operator $\mathbb{C}_{i}^{\mathrm{LCC}}$ in the phase $\omega_{i}$ is thus defined as the damagedincremental-secant operator $\mathbb{C}_{i}^{S \mathrm{D}}$ which is evaluated from the apparent stress and strain increments obtained from the residuals state as

$$
\langle\boldsymbol{\sigma}\rangle_{i_{n+1}}-\boldsymbol{\sigma}_{i_{n}}^{\mathrm{res}}\left(D_{i_{n+1}}\right)=\mathbb{C}_{i}^{\mathrm{SD}}:\langle\Delta \boldsymbol{\varepsilon}\rangle_{i}^{\mathrm{r}}
$$


with $\boldsymbol{\sigma}_{i_{n}}^{\text {res }}\left(D_{i_{n+1}}\right)$ defining the apparent residual stress that would be reached at configuration $t_{n}$ with the damage variable reached at configuration $t_{n+1}$, see Fig. 2(d), As previously explained, although the effective residual stress $\hat{\boldsymbol{\sigma}}_{i_{n}}^{\text {res }}$ does not depend on the variable $D_{i}$, the apparent residual stress does, i.e. $\boldsymbol{\sigma}_{i_{n}}^{\text {res }}\left(D_{i_{n+1}}\right)$ is not necessarily equal to $\boldsymbol{\sigma}_{i_{n}}^{\text {res }}\left(D_{i_{n}}\right)$ when the damage $D_{i}$ evolves.

Using these definitions of the linear operators, the set of Eqs. 45, 46 becomes

$$
\left\{\begin{array}{l}
\Delta \varepsilon_{\mathrm{M}}^{\mathrm{r}}=v_{0}\langle\Delta \varepsilon\rangle_{0}^{\mathrm{r}}+v_{\mathrm{I}}\langle\Delta \boldsymbol{\varepsilon}\rangle_{\mathrm{I}}^{\mathrm{r}} \text { and } \\
\boldsymbol{\sigma}_{\mathrm{M}_{n+1}}=v_{0}\langle\boldsymbol{\sigma}\rangle_{0_{n+1}}+v_{\mathrm{I}}\langle\boldsymbol{\sigma}\rangle_{\mathrm{I}_{n+1}} \quad \text { with } \\
\langle\Delta \boldsymbol{\varepsilon}\rangle_{\mathrm{I}}^{\mathrm{r}}=\mathbb{B}^{\epsilon}\left(\mathrm{I}, \mathbb{C}_{0}^{\mathrm{SD}}, \mathbb{C}_{\mathrm{I}}^{\mathrm{S} D}\right):\langle\Delta \boldsymbol{\varepsilon}\rangle_{0}^{\mathrm{r}},
\end{array}\right.
$$

where the average stress $\langle\boldsymbol{\sigma}\rangle_{i_{n+1}}$ at configuration time $t_{n+1}$ in the phase $\omega_{i}$ results from the constitutive box 47 .

The resolution of the set of equations $(53)$ follows the iterative process detailed in Section 3.4 .

\subsection{Phases incremental-secant operators}

The expressions of the damaged incremental-secant operators $\mathbb{C}_{i}^{\mathrm{SD}}$ are now particularised for the phase-field like fibre bundle damage model developed in Section 2.1 and considered in the inclusion phase $\omega_{\mathrm{I}}$, and for the non-local damage model developed in Section 2.2 and considered for the matrix phase $\omega_{0}$, as illustrated in Fig. 3. The debonding of fibre-matrix interfaces near the fibre breaking point and the debonding caused by transverse loading on the composites are captured by the damage in the matrix naturally. 


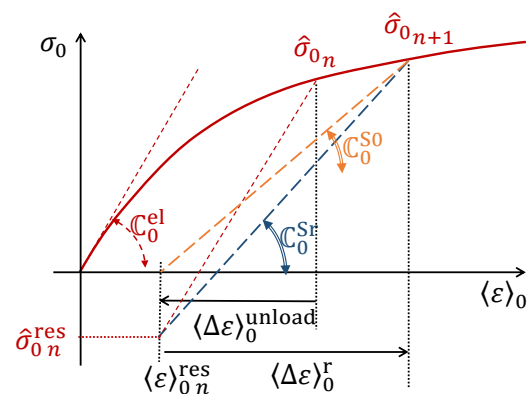

(a) Phase $\omega_{0}$

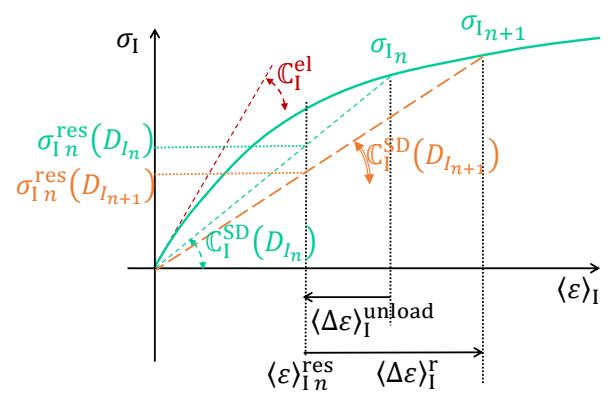

(b) Phase $\omega_{\text {I }}$

Figure 3: Particularisation of the LCC in the incremental-secant method for the (a) Damage-enhanced elasto-plastic matrix, with the definition in the effective stress space of the incremental-secant phase operator $\mathbb{C}_{0}^{\mathrm{Sr}}$ from the residual stress and of the incrementalsecant phase operator $\mathbb{C}_{0}^{S 0}$ from the zero-stress state; and for the (b) Damage-enhanced elastic fibre bundle.

\subsubsection{Matrix non-local damage model}

Damaged elastic material operator of the damage-enhanced elasto-plastic matrix material. Using the relation (33) governing the stress evolution in the matrix phase, the damaged fourth-order elastic operator $\mathbb{C}_{0}^{\mathrm{el} D}$ is evaluated from Eq. (34) as

$$
\mathbb{C}_{0}^{\mathrm{el} \mathrm{D}}\left(D_{0}\right)=\left(1-D_{0}\right) \mathbb{C}_{0}^{\mathrm{el}}=3\left(1-D_{0}\right) \kappa_{0} \mathbb{I}^{\mathrm{vol}}+2\left(1-D_{0}\right) \mu_{0} \mathbb{I}^{\mathrm{dev}},
$$

Incremental-secant operators of the damage-enhanced elasto-plastic matrix material. Following Eq. (33), the apparent residual stress reached upon virtual elastic unloading at configuration $t_{n}$ reads

$$
\boldsymbol{\sigma}_{0_{n}}^{\mathrm{res}}\left(D_{0_{n}}\right)=\left(1-D_{0_{n}}\right) \hat{\boldsymbol{\sigma}}_{0_{n}}^{\mathrm{res}}
$$


where $\hat{\boldsymbol{\sigma}}_{0_{n}}^{\text {res }}$ is the residual stress in the effective stress state, see Fig. 2(b). In the effective stress space, the incremental loading from the residual state to configuration $t_{n+1}$, see Fig. 2(d), defines the incremental-secant operator $\mathbb{C}_{0}^{\mathrm{Sr}}$ as

$$
\hat{\boldsymbol{\sigma}}_{0_{n+1}}-\hat{\boldsymbol{\sigma}}_{0_{n}}^{\mathrm{res}}=\mathbb{C}_{0}^{\mathrm{Sr}}:\langle\Delta \boldsymbol{\varepsilon}\rangle_{0}^{\mathrm{r}} .
$$

By considering the normal to the plastic flow from the residual state, see Appendix A.2.1, the incremental-secant operator $\mathbb{C}_{0}^{\mathrm{Sr}}$ is isotropic and can thus be written

$$
\mathbb{C}_{0}^{\mathrm{Sr}}=3 \kappa_{0} \mathbb{I}^{\mathrm{vol}}+2 \mu_{0}^{\mathrm{Sr}} \mathbb{I}^{\mathrm{dev}},
$$

where $\kappa_{0}$ is the elastic bulk modulus of the undamaged matrix material and $\mu_{0}^{\mathrm{Sr}}$ is the secant shear modulus which reads

$$
\mu_{0}^{\mathrm{Sr}}=\frac{1}{3} \frac{\sqrt{\frac{3}{2} \operatorname{dev}\left(\hat{\boldsymbol{\sigma}}_{0_{n+1}}-\hat{\boldsymbol{\sigma}}_{0_{n}}^{\mathrm{res}}\right): \operatorname{dev}\left(\hat{\boldsymbol{\sigma}}_{0_{n+1}}-\hat{\boldsymbol{\sigma}}_{0_{n}}^{\mathrm{res}}\right)}}{\sqrt{\frac{2}{3} \operatorname{dev}\left(\langle\Delta \boldsymbol{\varepsilon}\rangle_{0}^{\mathrm{r}}\right): \operatorname{dev}\left(\langle\Delta \boldsymbol{\varepsilon}\rangle_{0}^{\mathrm{r}}\right)}} .
$$

Because only first-statistical-moments are considered in this formulation, the incremental-secant method was shown to be over-stiff in its prediction [27, 31] and its predictive capabilities were improved in the case of hard inclusions when the residual stress in the matrix phase, $\hat{\sigma}_{0_{n}}^{\text {res }}$, was cancelled when defining the incremental-secant operator of the LCC [27, 31], see Fig. 3(a). Therefore, the residual of the matrix phase is removed in Eq. (56), which becomes

$$
\hat{\boldsymbol{\sigma}}_{0_{n+1}}=\mathbb{C}_{0}^{\mathrm{S} 0}:\langle\Delta \varepsilon\rangle_{0}^{\mathrm{r}},
$$

where

$$
\mathbb{C}_{0}^{\mathrm{S} 0}=3 \kappa_{0} \mathbb{I}^{\mathrm{vol}}+2 \mu_{0}^{\mathrm{S} 0} \mathbb{I}^{\mathrm{dev}},
$$


and where the increment shear modulus $(58)$ is rewritten as

$$
\mu_{0}^{\mathrm{S} 0}=\frac{1}{3} \frac{\sqrt{\frac{3}{2} \operatorname{dev}\left(\hat{\boldsymbol{\sigma}}_{0_{n+1}}\right): \operatorname{dev}\left(\hat{\boldsymbol{\sigma}}_{0_{n+1}}\right)}}{\sqrt{\frac{2}{3} \operatorname{dev}\left(\langle\Delta \boldsymbol{\varepsilon}\rangle_{0}^{\mathrm{r}}\right): \operatorname{dev}\left(\langle\Delta \boldsymbol{\varepsilon}\rangle_{0}^{\mathrm{r}}\right)}} .
$$

The incremental-secant operator is defined in the general form as

$$
\mathbb{C}_{0}^{\mathrm{S}}=3 \kappa_{0} \mathbb{I}^{\mathrm{vol}}+2 \mu_{0}^{\mathrm{S}} \mathbb{I}^{\mathrm{dev}}
$$

430 431

with $\mu_{0}^{\mathrm{S}}$ computed from either $(58)$ or 61 depending whether the residual is kept or not in the matrix phase.

Finally, in the apparent stress space, the incremental-secant damaged operator $\mathbb{C}_{0}^{\mathrm{SD}}$ is defined through Eq. 52 using the relation $\boldsymbol{\sigma}_{0_{n}}^{\text {res }}\left(D_{0_{n+1}}\right)=$ $\left(1-D_{0_{n+1}}\right) \hat{\boldsymbol{\sigma}}_{0_{n}}^{\mathrm{res}}$, Eq. 33 and Eq. 56 , which allow rewriting Eq. 52 as

$$
\mathbb{C}_{0}^{\mathrm{SD}}:\langle\Delta \boldsymbol{\varepsilon}\rangle_{0}^{\mathrm{r}}=\left(1-D_{0_{n+1}}\right)\left[\hat{\boldsymbol{\sigma}}_{0_{n+1}}-\hat{\boldsymbol{\sigma}}_{0_{n}}^{\mathrm{res}}\right]=\left(1-D_{0_{n+1}}\right) \mathbb{C}_{0}^{\mathrm{S}}:\langle\Delta \boldsymbol{\varepsilon}\rangle_{0}^{\mathrm{r}}
$$

As a result, the damaged incremental-secant operator reads

$$
\mathbb{C}_{0}^{\mathrm{SD}}=3\left(1-D_{0_{n+1}}\right) \kappa_{0} \mathbb{I}^{\mathrm{vol}}+2\left(1-D_{0_{n+1}}\right) \mu_{0}^{\mathrm{S}} \mathbb{I}^{\mathrm{dev}}=3 \kappa_{0}^{\mathrm{D}} \mathbb{I}^{\mathrm{vol}}+2 \mu_{0}^{\mathrm{SD}} \mathbb{I}^{\mathrm{dev}}
$$

${ }_{432}$ with $\kappa_{0}^{\mathrm{D}}=\left(1-D_{0_{n+1}}\right) \kappa_{0}$ and $\mu_{0}^{\mathrm{SD}}=\left(1-D_{0_{n+1}}\right) \mu_{0}^{\mathrm{S}}$.

\subsubsection{Embedded fibre bundle damage model}

The stress tensor of the damaged elastic fibre bundle results from Eq. (24) and reads

$$
\langle\boldsymbol{\sigma}\rangle_{\mathrm{I}}=\mathbb{C}_{\mathrm{I}}^{\mathrm{D}}\left(D_{\mathrm{I}}\right):\langle\varepsilon\rangle_{\mathrm{I}}
$$

with the damaged elastic operator 21 . 
Damaged elastic material operator of the fibre bundle material. The fibre bundle damaged fourth-order elastic operator $\mathbb{C}_{\mathrm{I}}^{\text {el } \mathrm{D}}$ is directly evaluated from Eqs. (21) and (24) as

$$
\mathbb{C}_{\mathrm{I}}^{\mathrm{el} \mathrm{D}}\left(D_{\mathrm{I}}\right)=\mathbb{C}_{\mathrm{I}}^{\mathrm{D}}\left(D_{\mathrm{I}}\right),
$$

435

Incremental-secant operators of the damage-enhanced fibre bundle material.

In the absence of plastic-flow in the fibre bundle, the residual stress tensors from the virtual elastic-unloading at configuration $t_{n}$ are defined following Eq. 655) for the two damage configurations

$$
\boldsymbol{\sigma}_{\mathrm{I}_{n}}^{\mathrm{res}}\left(D_{\mathrm{I}_{n}}\right)=\mathbb{C}_{\mathrm{I}}^{\mathrm{D}}\left(D_{\mathrm{I}_{n}}\right):\langle\varepsilon\rangle_{\mathrm{I}_{n}}^{\mathrm{res}} \quad \text { and } \quad \boldsymbol{\sigma}_{\mathrm{I}_{n}}^{\mathrm{res}}\left(D_{\mathrm{I}_{n+1}}\right)=\mathbb{C}_{\mathrm{I}}^{\mathrm{D}}\left(D_{\mathrm{I}_{n+1}}\right):\langle\varepsilon\rangle_{\mathrm{I}_{n}}^{\mathrm{res}},
$$

as illustrated in Fig. 3(b),

Equation (52) defines the fourth-order incremental-secant operator $\mathbb{C}_{I}^{\mathrm{SD}}$ of the fibre bundle, with

$$
\begin{aligned}
\mathbb{C}_{\mathrm{I}}^{\mathrm{SD}}:\langle\Delta \varepsilon\rangle_{\mathrm{I}}^{\mathrm{r}} & =\langle\boldsymbol{\sigma}\rangle_{\mathrm{I}_{n+1}}-\sigma_{\mathrm{I}_{n}}^{\mathrm{res}}\left(D_{\mathrm{I}_{n+1}}\right)=\mathbb{C}_{\mathrm{I}}^{\mathrm{D}}\left(D_{\mathrm{I}_{n+1}}\right):\left[\langle\boldsymbol{\varepsilon}\rangle_{\mathrm{I}_{n+1}}-\langle\varepsilon\rangle_{\mathrm{I}_{n}}^{\mathrm{res}}\right] \\
& =\mathbb{C}_{\mathrm{I}}^{\mathrm{D}}\left(D_{\mathrm{I}_{n+1}}\right):\langle\Delta \varepsilon\rangle_{\mathrm{I}}^{\mathrm{r}},
\end{aligned}
$$

and

$$
\mathbb{C}_{\mathrm{I}}^{\mathrm{SD}}\left(D_{\mathrm{I}}\right)=\mathbb{C}_{\mathrm{I}}^{\mathrm{el} \mathrm{D}}\left(D_{\mathrm{I}}\right)=\mathbb{C}_{\mathrm{I}}^{\mathrm{D}}\left(D_{\mathrm{I}}\right),
$$

where $D_{\mathrm{I}}=D_{\mathrm{I}_{n+1}}$ is the damage reached during the reloading to configuration $t_{n+1}$, which is evaluated through Eqs. (7) and (9). 
441

442

\subsubsection{Linearisation of the MFH equations}

Combining the first and last equations of the set (53) and using the M-T assumption 43) yield

$$
\Delta \varepsilon_{\mathrm{M}}^{\mathrm{r}}=v_{0}\left[\mathbb{I}+\mathbb{S}\left(\mathrm{I}, \mathbb{C}_{0}^{\mathrm{S} D}\right):\left[\left(\mathbb{C}_{0}^{\mathrm{S} D}\right)^{-1}: \mathbb{C}_{\mathrm{I}}^{\mathrm{SD}}-\mathbb{I}\right]\right]:\langle\Delta \varepsilon\rangle_{\mathrm{I}}^{\mathrm{r}}+v_{\mathrm{I}}\langle\Delta \varepsilon\rangle_{\mathrm{I}}^{\mathrm{r}}
$$

which is satisfied for $\mathbf{F}\left(\langle\Delta \varepsilon\rangle_{\mathrm{I}}^{\mathrm{r}},\langle\Delta \varepsilon\rangle_{0}^{\mathrm{r}} ; \Delta \varepsilon_{\mathrm{M}}^{\mathrm{r}}, \tilde{p}_{0}, d_{\mathrm{I}}\right)=0$ with

$$
\mathbf{F}=\mathbb{C}_{0}^{\mathrm{SD}}:\left[\langle\Delta \varepsilon\rangle_{\mathrm{I}}^{\mathrm{r}}-\frac{1}{v_{0}} \mathbb{S}^{-1}\left(\mathrm{I}, \mathbb{C}_{0}^{\mathrm{SD}}\right):\left(\langle\Delta \varepsilon\rangle_{\mathrm{I}}^{\mathrm{r}}-\Delta \varepsilon_{\mathrm{M}}^{\mathrm{r}}\right)\right]-\mathbb{C}_{\mathrm{I}}^{\mathrm{SD}}:\langle\Delta \varepsilon\rangle_{\mathrm{I}}^{\mathrm{r}}
$$

443

$$
\begin{aligned}
\delta \mathbf{F}= & \frac{\partial \mathbf{F}}{\partial\langle\Delta \varepsilon\rangle_{\mathrm{I}}^{\mathrm{r}}}: \delta\langle\Delta \varepsilon\rangle_{\mathrm{I}}^{\mathrm{r}}+\frac{\partial \mathbf{F}}{\partial\langle\Delta \varepsilon\rangle_{0}^{\mathrm{r}}}: \frac{\partial\langle\Delta \varepsilon\rangle_{0}^{\mathrm{r}}}{\partial\langle\Delta \varepsilon\rangle_{\mathrm{I}}^{\mathrm{r}}} \delta\langle\Delta \varepsilon\rangle_{\mathrm{I}}^{\mathrm{r}}+ \\
& \frac{\partial \mathbf{F}}{\partial \Delta \varepsilon_{\mathrm{M}}^{\mathrm{r}}}: \delta \Delta \varepsilon_{\mathrm{M}}^{\mathrm{r}}+\frac{\partial \mathbf{F}}{\partial \tilde{p}_{0}} \delta \tilde{p}_{0}+\frac{\partial \mathbf{F}}{\partial d_{\mathrm{I}}} \delta d_{\mathrm{I}} .
\end{aligned}
$$

Because of the first equation of the set (53), at constant $\Delta \varepsilon_{\mathrm{M}}^{\mathrm{r}}, \tilde{p}_{0}, d_{\mathrm{I}}$, one has

$$
\frac{\partial\langle\Delta \varepsilon\rangle_{0}^{\mathrm{r}}}{\partial\langle\Delta \varepsilon\rangle_{\mathrm{I}}^{\mathrm{r}}}=-\frac{v_{\mathrm{I}}}{v_{0}} \mathbb{I}
$$

and defining the Jacobian

$$
\mathbb{J}=\frac{\partial \mathbf{F}}{\langle\Delta \varepsilon\rangle_{\mathrm{I}}^{\mathrm{r}}}-\frac{v_{\mathrm{I}}}{v_{0}} \frac{\partial \mathbf{F}}{\langle\Delta \varepsilon\rangle_{0}^{\mathrm{r}}}
$$

Eq. 72 is rewritten as

$$
\delta \mathbf{F}=\mathbb{J}: \delta\langle\Delta \varepsilon\rangle_{\mathrm{I}}^{\mathrm{r}}+\frac{\partial \mathbf{F}}{\partial \Delta \varepsilon_{\mathrm{M}}^{\mathrm{r}}}: \delta \Delta \varepsilon_{\mathrm{M}}^{\mathrm{r}}+\frac{\partial \mathbf{F}}{\partial \tilde{p}_{0}}: \delta \tilde{p}_{0}+\frac{\partial \mathbf{F}}{\partial d_{\mathrm{I}}}: \delta d_{\mathrm{I}} .
$$

${ }_{444}$ The explicit expressions of the derivatives are reported in Appendix B.1. 


\subsubsection{MFH iterative resolution}

For given kinematics variables $\Delta \varepsilon_{\mathrm{M}}^{\mathrm{r}}, \tilde{p}_{0}$ and $d_{\mathrm{I}}$ resulting from the finite element resolution, the resolution of the set of $\mathrm{MFH}$ equations restated by Eq. (71) follows an iterative Newton-Raphson process in the unknown $\langle\Delta \varepsilon\rangle_{\mathrm{I}}^{\mathrm{r}}$, with the linearisation 75 rewritten as

$$
\delta \mathbf{F}=\mathbb{J}: \delta\langle\Delta \varepsilon\rangle_{\mathrm{I}}^{\mathrm{r}}
$$

\subsection{Algorithmic operators of the homogenised behaviour}

To be complete, we present the algorithmic operators of the homogenised behaviour with respect to the kinematics variables $\Delta \varepsilon_{\mathrm{M}}^{\mathrm{r}}, \tilde{p}_{0}$ and $d_{\mathrm{I}}$. Indeed, in this work the damage evolution in the matrix and fibre phases are governed respectively by a non-local and a phase-field forms, respectively Eqs. (38) and $(30)$, and both $\tilde{p}_{0}$ and $d_{\mathrm{I}}$ result from the resolution of the finite elements discretisation as detailed in the next Section.

First, once the MFH equations are solved for given kinematics variables $\Delta \varepsilon_{\mathrm{M}}^{\mathrm{r}}, \tilde{p}_{0}$ and $d_{\mathrm{I}}$, their effects on the phases response can be evaluated from Eq. (75) by considering that at equilibrium $\delta \mathbf{F}=\mathbf{0}$ and $\Delta \varepsilon_{\mathrm{M}}^{\mathrm{r}}=v_{\mathrm{I}}\langle\Delta \varepsilon\rangle_{\mathrm{I}}^{\mathrm{r}}+v_{0}\langle\Delta \varepsilon\rangle_{0}^{\mathrm{r}}$. The effect of the macro-scale strain tensor $\Delta \varepsilon_{\mathrm{M}}^{\mathrm{r}}$ on the phases response reads

$$
\frac{\partial\langle\Delta \varepsilon\rangle_{\mathrm{I}}^{\mathrm{r}}}{\partial \Delta \varepsilon_{\mathrm{M}}^{\mathrm{r}}}=-\mathbb{J}^{-1}: \frac{\partial \mathbf{F}}{\partial \Delta \varepsilon_{\mathrm{M}}^{\mathrm{r}}} \text {, and } \frac{\partial\langle\Delta \varepsilon\rangle_{0}^{\mathrm{r}}}{\partial \Delta \varepsilon_{\mathrm{M}}^{\mathrm{r}}}=\frac{1}{v_{0}} \mathbb{I}+\frac{v_{\mathrm{I}}}{v_{0}} \mathbb{J}^{-1}: \frac{\partial \mathbf{F}}{\partial \Delta \varepsilon_{\mathrm{M}}^{\mathrm{r}}} \text {. }
$$

Similarly, the effects of the non-local strain and auxiliary damage variables read

$$
\begin{aligned}
& \frac{\partial\langle\Delta \boldsymbol{\varepsilon}\rangle_{\mathrm{I}}^{\mathrm{r}}}{\partial \tilde{p}_{0}}=-\mathbb{J}^{-1}: \frac{\partial \mathbf{F}}{\partial \tilde{p}_{0}}, \quad \frac{\partial\langle\Delta \boldsymbol{\varepsilon}\rangle_{0}^{\mathrm{r}}}{\partial \tilde{p}_{0}}=\frac{v_{\mathrm{I}}}{v_{0}} \mathbb{J}^{-1}: \frac{\partial \mathbf{F}}{\partial \tilde{p}_{0}}, \\
& \frac{\partial\langle\Delta \varepsilon\rangle_{\mathrm{I}}^{\mathrm{r}}}{\partial d_{\mathrm{I}}}=-\mathbb{J}^{-1}: \frac{\partial \mathbf{F}}{\partial d_{\mathrm{I}}}, \quad \text { and } \quad \frac{\partial\langle\Delta \varepsilon\rangle_{0}^{\mathrm{r}}}{\partial d_{\mathrm{I}}}=\frac{v_{\mathrm{I}}}{v_{0}} \mathbb{J}^{-1}: \frac{\partial \mathbf{F}}{\partial d_{\mathrm{I}}} \text {. }
\end{aligned}
$$


Then, the linearisation of the homogenised stress tensor given by Eq. (53) can be evaluated

$$
\begin{aligned}
\delta \boldsymbol{\sigma}_{\mathrm{M}}= & \left(v_{\mathrm{I}} \mathbb{C}_{\mathrm{I}}^{\varepsilon \varepsilon}: \frac{\partial\langle\varepsilon\rangle_{\mathrm{I}}^{\mathrm{r}}}{\partial \varepsilon_{\mathrm{M}}^{\mathrm{r}}}+v_{0} \mathbb{C}_{0}^{\varepsilon \varepsilon}: \frac{\partial\langle\varepsilon\rangle_{0}^{\mathrm{r}}}{\partial \varepsilon_{\mathrm{M}}^{\mathrm{r}}}\right): \delta \varepsilon_{\mathrm{M}}^{\mathrm{r}}+ \\
& \left(v_{\mathrm{I}} \mathbb{C}_{\mathrm{I}}^{\varepsilon \varepsilon}: \frac{\partial\langle\varepsilon\rangle_{\mathrm{I}}^{\mathrm{r}}}{\partial d_{\mathrm{I}}}+v_{0} \mathbb{C}_{0}^{\varepsilon \varepsilon}: \frac{\partial\langle\varepsilon\rangle_{0}^{\mathrm{r}}}{\partial d_{\mathrm{I}}}+v_{\mathrm{I}} \boldsymbol{C}_{\mathrm{I}}^{\varepsilon d}\right) \delta d_{\mathrm{I}}+ \\
& \left(v_{\mathrm{I}} \mathbb{C}_{\mathrm{I}}^{\varepsilon \varepsilon}: \frac{\partial\langle\varepsilon\rangle_{\mathrm{I}}^{\mathrm{r}}}{\partial \tilde{p}_{0}}+v_{0} \mathbb{C}_{0}^{\varepsilon \varepsilon}: \frac{\partial\langle\varepsilon\rangle_{0}^{\mathrm{r}}}{\partial \tilde{p}_{0}}+v_{0} \boldsymbol{C}_{0}^{\varepsilon \tilde{p}}\right) \delta \tilde{p}_{0}
\end{aligned}
$$

where the fibre bundle material operators $\mathbb{C}_{\mathrm{I}}^{\varepsilon \varepsilon}=\frac{\partial\langle\boldsymbol{\sigma}\rangle_{\mathrm{I}}}{\partial\langle\boldsymbol{\varepsilon}\rangle_{\mathrm{I}}}$ and $\boldsymbol{C}_{\mathrm{I}}^{\varepsilon d}=\frac{\partial\langle\boldsymbol{\sigma}\rangle_{\mathrm{I}}}{\partial d_{\mathrm{I}}}$ are given in Appendix A.1.1 and the matrix material operators $\mathbb{C}_{0}^{\varepsilon \varepsilon}=\frac{\partial\langle\boldsymbol{\sigma}\rangle_{0}}{\partial\langle\boldsymbol{\varepsilon}\rangle_{0}}$ and $\boldsymbol{C}_{0}^{\varepsilon \tilde{p}}=\frac{\partial\langle\boldsymbol{\sigma}\rangle_{0}}{\partial \tilde{p}_{0}}$ are given in Appendix A.2.2. The derivatives of the phases average strain tensors result from the MFH resolution and are given in Eqs. (77,79). Finally, the different terms of Eq. (80) are denoted as

$$
\begin{aligned}
\mathbb{C}_{\mathrm{M}}^{\varepsilon \varepsilon} & =v_{\mathrm{I}} \mathbb{C}_{\mathrm{I}}^{\varepsilon \varepsilon}: \frac{\partial\langle\varepsilon\rangle_{\mathrm{I}}^{\mathrm{r}}}{\partial \varepsilon_{\mathrm{M}}^{\mathrm{r}}}+v_{0} \mathbb{C}_{0}^{\varepsilon \varepsilon}: \frac{\partial\langle\varepsilon\rangle_{0}^{\mathrm{r}}}{\partial \varepsilon_{\mathrm{M}}^{\mathrm{r}}}, \\
\boldsymbol{C}_{\mathrm{M}}^{\varepsilon d} & =v_{\mathrm{I}} \mathbb{C}_{\mathrm{I}}^{\varepsilon \varepsilon}: \frac{\partial\langle\varepsilon\rangle_{\mathrm{I}}^{\mathrm{r}}}{\partial d_{\mathrm{I}}}+v_{0} \mathbb{C}_{0}^{\varepsilon \varepsilon}: \frac{\partial\langle\varepsilon\rangle_{0}^{\mathrm{r}}}{\partial d_{\mathrm{I}}}+v_{\mathrm{I}} \boldsymbol{C}_{\mathrm{I}}^{\varepsilon d}, \quad \text { and } \\
\boldsymbol{C}_{\mathrm{M}}^{\varepsilon \tilde{p}} & =v_{\mathrm{I}} \mathbb{C}_{\mathrm{I}}^{\varepsilon \varepsilon}: \frac{\partial\langle\varepsilon\rangle_{\mathrm{I}}^{\mathrm{r}}}{\partial \tilde{p}_{0}}+v_{0} \mathbb{C}_{0}^{\varepsilon \varepsilon}: \frac{\partial\langle\varepsilon\rangle_{0}^{\mathrm{r}}}{\partial \tilde{p}_{0}}+v_{0} \boldsymbol{C}_{0}^{\varepsilon \tilde{p}},
\end{aligned}
$$

allowing to write down $\delta \boldsymbol{\sigma}_{\mathrm{M}}=\mathbb{C}_{\mathrm{M}}^{\varepsilon \varepsilon}: \delta \boldsymbol{\varepsilon}_{\mathrm{M}}^{\mathrm{r}}+\boldsymbol{C}_{\mathrm{M}}^{\varepsilon d} \delta d_{\mathrm{I}}+\boldsymbol{C}_{\mathrm{M}}^{\varepsilon \tilde{p}} \delta \tilde{p}_{0}$.

In order to solve the coupled system of equations, the derivatives of the different terms involved in Eq. (30) have also to be evaluated at the level of the composite material, yielding

$$
\begin{aligned}
C_{\mathrm{M}}^{\psi \varepsilon} & =\frac{\partial\left(-\frac{l_{\mathrm{I}}}{G_{c}} \frac{\partial \psi_{\mathrm{I}}^{+}}{\partial d_{\mathrm{I}}}\right)}{\partial \varepsilon_{\mathrm{M}}^{\mathrm{r}}}=C_{\mathrm{I}}^{\psi \varepsilon}: \frac{\partial\langle\varepsilon\rangle_{\mathrm{I}}^{\mathrm{r}}}{\partial \varepsilon_{\mathrm{M}}^{\mathrm{r}}} \\
C_{\mathrm{M}}^{\psi d} & =\frac{d\left(-\frac{l_{\mathrm{I}}}{G_{c}} \frac{\partial \psi_{\mathrm{I}}^{+}}{\partial d_{\mathrm{I}}}\right)}{d d_{\mathrm{I}}}=\boldsymbol{C}_{\mathrm{I}}^{\psi \varepsilon}: \frac{\partial\langle\varepsilon\rangle_{\mathrm{I}}^{\mathrm{r}}}{\partial d_{\mathrm{I}}}+C_{\mathrm{I}}^{\psi d}, \text { and } \\
C_{\mathrm{M}}^{\psi \tilde{p}} & =\frac{\partial\left(-\frac{l_{\mathrm{I}}}{G_{c}} \frac{\partial \psi_{\mathrm{I}}^{+}}{\partial d_{\mathrm{I}}}\right)}{\partial \tilde{p}_{0}}=\boldsymbol{C}_{\mathrm{I}}^{\psi \varepsilon}: \frac{\partial\langle\varepsilon\rangle_{\mathrm{I}}^{\mathrm{r}}}{\partial \tilde{p}_{0}}
\end{aligned}
$$


[ where $\boldsymbol{C}_{\mathrm{I}}^{\psi \varepsilon}=\frac{\partial\left(-\frac{l_{\mathrm{I}}}{G_{c}} \frac{\partial \psi_{\mathrm{I}}^{+}}{\partial d_{\mathrm{I}}}\right)}{\partial\langle\varepsilon\rangle_{\mathrm{I}}}$ and $C_{\mathrm{I}}^{\psi d}=\frac{\partial\left(-\frac{l_{\mathrm{I}}}{G_{c}} \frac{\partial \psi_{\mathrm{I}}^{+}}{\partial d_{\mathrm{I}}}\right)}{\partial d_{\mathrm{I}}}$ are given in Appendix A.1.1.

Finally, the terms of the coupled system (38) also have to be linearised at the composite level, yielding

$$
\begin{aligned}
\boldsymbol{C}_{\mathrm{M}}^{p \varepsilon} & =\frac{\partial p_{0}}{\partial \varepsilon_{\mathrm{M}}^{\mathrm{r}}}=\boldsymbol{C}_{0}^{p \varepsilon}: \frac{\partial\langle\varepsilon\rangle_{0}^{\mathrm{r}}}{\partial \varepsilon_{\mathrm{M}}^{\mathrm{r}}}, \\
\boldsymbol{C}_{\mathrm{M}}^{p d} & =\frac{\partial p_{0}}{\partial d_{\mathrm{I}}}=\boldsymbol{C}_{0}^{p \varepsilon}: \frac{\partial\langle\varepsilon\rangle_{0}^{\mathrm{r}}}{\partial d_{\mathrm{I}}}, \text { and } \\
\boldsymbol{C}_{\mathrm{M}}^{p \tilde{p}} & =\frac{\mathrm{d} p_{0}}{\mathrm{~d} \tilde{p}_{0}}=\frac{\partial p_{0}}{\partial\langle\varepsilon\rangle_{0}^{\mathrm{r}}}: \frac{\partial\langle\varepsilon\rangle_{0}^{\mathrm{r}}}{\partial \tilde{p}_{0}}+\frac{\partial p_{0}}{\partial \tilde{p}_{0}}=\boldsymbol{C}_{0}^{p \varepsilon}: \frac{\partial\langle\varepsilon\rangle_{0}^{\mathrm{r}}}{\partial \tilde{p}_{0}}+C_{0}^{p \tilde{p}},
\end{aligned}
$$

where $\boldsymbol{C}_{0}^{p \varepsilon}=\frac{\partial p_{0}}{\partial\langle\varepsilon\rangle_{0}}$ and $C_{0}^{p \tilde{p}}=\frac{\partial p_{0}}{\partial \tilde{p}_{0}}$ are given in Appendix A.2.2.

\section{Finite element discretisation of the phase-field non-local damage MFH}

In this section, starting from the strong form of the linear momentum conservation equation at the composite level completed by the phase-field and non-local damage auxiliary equations, we derive the finite element discretisation of the homogenised behaviour.

\subsection{Strong form}

The problem is limited to small deformations and static analyses. The governing equations at the homogenised behaviour level read

$$
\begin{aligned}
\nabla \cdot \boldsymbol{\sigma}_{\mathrm{M}}+\boldsymbol{f}=\mathbf{0} & \text { for composite, } \\
d_{\mathrm{I}}-\nabla \cdot\left(\boldsymbol{c}_{\mathrm{I}} \cdot \nabla d_{\mathrm{I}}\right)-\frac{l_{\mathrm{I}}}{G_{c}} \varepsilon\left\langle\dot{d}_{\mathrm{I}}\right\rangle_{-}=-\frac{l_{\mathrm{I}}}{G_{c}} \psi_{\mathrm{I}, d_{\mathrm{I}}}^{+} & \text {for fibre bundle, } \\
\tilde{p}_{0}-\nabla \cdot\left(\boldsymbol{c}_{0} \cdot \nabla \tilde{p}_{0}\right)=p_{0}, & \text { for matrix }
\end{aligned}
$$


The first equation corresponds to the linear momentum equilibrium equation of the composite material, with $\boldsymbol{f}$ the applied volume force vector. The second equation is the phase-field formulation (30), which refers to the damage evolution of the fibre bundle phase in an average sense. Neither the auxiliary variable $d_{\mathrm{I}}$ nor the damage variable $D_{\mathrm{I}}$ correspond to the phase volume average, but they are constructed as uniform on the phase for a given macro-scale material point. The squared characteristic lengths matrix $\boldsymbol{c}_{\mathrm{I}}$ corresponds to the matrix $\operatorname{diag}\left(0,0, l_{\mathrm{I}}^{2}\right)$, with the last entry referring to the longitudinal direction of the fibres rotated from the material principal coordinates to the current fibre bundle direction. Finally, the third equation results from the non-local damage formulation (38), which refers to the damage evolution in the matrix phase. In particular, $\tilde{p}_{0}$ and $p_{0}$ are homogenised representations, but not volume average values, of respectively the non-local and local accumulated plastic strain of the matrix material, and $\boldsymbol{c}_{0}$ is a rotation of the squared characteristic lengths matrix $\operatorname{diag}\left(l_{10}^{2}, l_{20}^{2}, l_{30}^{2}\right)$. In this last expression written in the material principal coordinates, the index '3' refers to the longitudinal direction of the fibre bundles, while the two other indices refer to the transverse direction characterised by smaller characteristic lengths because the damage propagation is blocked to the presence of the other fibres. Standard Neumann boundary conditions

$$
\boldsymbol{\sigma} \cdot \boldsymbol{n}=\boldsymbol{T}, \quad \text { on } \quad \Gamma_{T},
$$

with the surface traction $\boldsymbol{T}$ and Dirichlet boundary conditions on $\Gamma_{u}$ are applied to the first set of partial differential equations (PDE) (90). For the phase-field formulations (91) and implicit gradient formulation (92), homo- 
506

geneous Neumann boundary conditions are applied:

$$
\begin{aligned}
& \left(\boldsymbol{c}_{\mathrm{I}} \cdot \nabla d_{\mathrm{I}}\right) \cdot \boldsymbol{n}=0, \text { on } \partial \Omega, \text { and } \\
& \left(\boldsymbol{c}_{0} \cdot \nabla \tilde{p}_{0}\right) \cdot \boldsymbol{n}=0 \text { on } \partial \Omega .
\end{aligned}
$$

507

\subsection{Weak formulation}

The weak form of the set of Eqs. (90, 92) is established using suitable weight functions defined in the $n+2$-dimensional spaces, with $n$ the spatial dimension:

$\boldsymbol{w}_{u} \in\left[C^{0}\right]^{n} \quad$ The weight function of the displacement field, $w_{d} \in\left[C^{0}\right] \quad$ The weight function of the auxiliary damage field of fibre bundle, $w_{\tilde{p}} \in\left[C^{0}\right] \quad$ The weight function of non-local accumulated plastic strain field of the matrix phase.

Multiplying the weight functions respectively with their corresponding PDE (90, 91, 92), integrating the results over the domain $\Omega$ and applying the divergence theorem along with the boundary conditions (93.95) allows stating the weak form as finding the fields $\left(\boldsymbol{u}, d_{\mathrm{I}}, \tilde{p}_{0}\right)$, with $\boldsymbol{u}$ the displacement field, such that

$$
\begin{aligned}
\int_{\Omega}\left[\nabla \boldsymbol{w}_{u}\right]^{\mathrm{T}}: \boldsymbol{\sigma}_{\mathrm{M}} \mathrm{d} V-\int_{\Gamma_{T}} \boldsymbol{w}_{u} \cdot \boldsymbol{T} \mathrm{d} S & =\int_{\Omega} \boldsymbol{w}_{u} \cdot \boldsymbol{f} \mathrm{d} V \\
\int_{\Omega}\left(w_{d} d_{\mathrm{I}}+\nabla w_{d} \cdot \boldsymbol{c}_{\mathrm{I}} \cdot \nabla d_{\mathrm{I}}-w_{d} \frac{l_{\mathrm{I}}}{G_{c}} \varepsilon\left\langle\dot{d}_{\mathrm{I}}\right\rangle_{-}\right) \mathrm{d} V & =-\int_{\Omega} w_{d} \frac{l_{\mathrm{I}}}{G_{c}} \psi_{\mathrm{I}, d_{\mathrm{I}}}^{+}(\boldsymbol{u}) \mathrm{d} V \\
\int_{\Omega}\left(w_{\tilde{p}} \tilde{p}_{0}+\nabla w_{\tilde{p}} \cdot \boldsymbol{c}_{0} \cdot \nabla \tilde{p}_{0}\right) \mathrm{d} V & =\int_{\Omega} w_{\tilde{p}} p_{0} \mathrm{~d} V
\end{aligned}
$$

516 for all kinematically admissible weight functions $\left(\boldsymbol{w}_{u}, w_{d}, w_{\tilde{p}}\right)$. 
Anticipating on the Newton Raphson resolution of the upcoming finiteelement resolution, the set of Eqs. (97,99) is linearised at iteration $i$ of the configurations increment $\left[t_{n}, t_{n+1}\right]$ as

$$
\begin{array}{r}
\int_{\Omega}\left[\nabla \boldsymbol{w}_{u}\right]^{\mathrm{T}}: \delta \boldsymbol{\sigma}_{\mathrm{M}_{n+1}}^{i+1} \mathrm{~d} V= \\
\int_{\Omega} \boldsymbol{w}_{u} \cdot \boldsymbol{f}_{n+1} \mathrm{~d} V+\int_{\Gamma_{T}} \boldsymbol{w}_{u} \cdot \boldsymbol{T}_{n+1} \mathrm{~d} S-\int_{\Omega}\left[\nabla \boldsymbol{w}_{u}\right]^{\mathrm{T}}: \boldsymbol{\sigma}_{\mathrm{M}_{n+1}}^{i} \mathrm{~d} V,
\end{array}
$$

for the first equation; substituting $\dot{d}_{\mathrm{I}}$ by $d_{\mathrm{I}}-d_{\mathrm{I} n}$ since the purpose of the term is solely to ensure irreversibility of the damaging process, as

$$
\begin{array}{r}
\int_{\Omega} w_{d} \delta\left[\frac{l_{\mathrm{I}}}{G_{c}} \psi_{\mathrm{I}, d_{\mathrm{I}}}^{+i+1}\right] \mathrm{d} V+\int_{\Omega}\left[w_{d} \delta d_{\mathrm{I} n+1}^{i+1}+\nabla w_{d} \cdot \boldsymbol{c}_{\mathrm{I}} \cdot \nabla \delta d_{\mathrm{I} n+1}^{i+1} \mathrm{~d} V\right. \\
\left.-\int_{\Omega} w_{d} \frac{l_{\mathrm{I}}}{G_{c}} \varepsilon\left\langle\operatorname{sign}\left(d_{\mathrm{I} n+1}^{i}-d_{\mathrm{I} n}\right)\right\rangle_{-} \delta d_{\mathrm{I} n+1}^{i+1}\right] \mathrm{d} V=-\int_{\Omega} w_{d} \frac{l_{\mathrm{I}}}{G_{c}} \psi_{\mathrm{I}, d_{\mathrm{I}}}^{+i} \mathrm{~d} V- \\
\int_{\Omega}\left[w_{d} d_{\mathrm{I} n+1}^{i}+\nabla w_{d} \cdot \boldsymbol{c}_{\mathrm{I}} \cdot \nabla d_{\mathrm{I} n+1}^{i}-w_{d} \frac{l_{\mathrm{I}}}{G_{c}} \varepsilon\left\langle d_{\mathrm{I} n+1}^{i}-d_{\mathrm{I} n}\right\rangle_{-}\right] \mathrm{d} V,
\end{array}
$$

for the second equation where $\operatorname{sign}(\bullet)$ is the sign function, and

$$
\begin{array}{r}
-\int_{\Omega} w_{\tilde{p}} \delta p_{0 n+1}^{i+1} \mathrm{~d} V+\int_{\Omega}\left(w_{\tilde{p}} \delta \tilde{p}_{0 n+1}^{i+1}+\nabla w_{\tilde{p}} \cdot \boldsymbol{c}_{0} \cdot \nabla \delta \tilde{p}_{0 n+1}^{i+1}\right) \mathrm{d} V= \\
\int_{\Omega} w_{\tilde{p}} p_{0 n+1}^{i} \mathrm{~d} V-\int_{\Omega}\left(w_{\tilde{p}} \tilde{p}_{0 n+1}^{i}+\nabla w_{\tilde{p}} \cdot \boldsymbol{c}_{0} \cdot \nabla \tilde{p}_{0 n+1}^{i}\right) \mathrm{d} V,
\end{array}
$$

for the third equation.

\subsection{Finite element implementation - Discretisation and incremental-iterative formulation}

The domain $\Omega$ is discretized into elements $\Omega_{\mathrm{e}}$, and the displacement field $\boldsymbol{u}$, the auxiliary damage field $d_{\mathrm{I}}$, and the non-local accumulated plastic stain field $\tilde{p}_{0}$ are interpolated in each element using their respective shape function matrices $\boldsymbol{N}_{u}, \boldsymbol{N}_{d}$ and $\boldsymbol{N}_{\tilde{p}}$ as follows:

$$
\boldsymbol{u}=\boldsymbol{N}_{u} \boldsymbol{U}, \quad d_{\mathrm{I}}=\boldsymbol{N}_{d} \boldsymbol{d} \quad \text { and } \quad \tilde{p}_{0}=\boldsymbol{N}_{\tilde{p}} \tilde{\boldsymbol{p}}
$$


where the vectors $\boldsymbol{U}, \tilde{\boldsymbol{p}}$ and $\boldsymbol{d}$ contain the assembled nodal values of the displacement field, of the auxiliary damage field, and of the non-local accumulated plastic strain field, respectively. The fields gradients directly arise from

$$
\boldsymbol{\varepsilon}_{\mathrm{M}}=\boldsymbol{B}_{u} \boldsymbol{U}, \quad \nabla d_{\mathrm{I}}=\boldsymbol{B}_{d} \boldsymbol{d} \text { and } \nabla \tilde{p}_{0}=\boldsymbol{B}_{\tilde{p}} \tilde{\boldsymbol{p}},
$$

where $\boldsymbol{B}_{u}, \boldsymbol{B}_{d}$, and $\boldsymbol{B}_{\tilde{p}}$ are the matrix gradient operators of the displacement field, auxiliary damage field, and non-local accumulated plastic strain field, respectively. Similarly, the weight functions are interpolated using the same shape functions, yielding

$$
\boldsymbol{w}_{u}=\boldsymbol{N}_{u} \delta \boldsymbol{U}, \quad w_{d}=\boldsymbol{N}_{d} \delta \boldsymbol{d} \quad \text { and } \quad w_{\tilde{p}}=\boldsymbol{N}_{\tilde{p}} \delta \tilde{\boldsymbol{p}},
$$

where $\delta \boldsymbol{U}, \delta \boldsymbol{d}$ and $\delta \tilde{\boldsymbol{p}}$ are arbitrary vectors fulfilling the essential boundary conditions.

Therefore, using Eqs. (103,105), and the arbitrary nature of $\delta \boldsymbol{U}, \delta \boldsymbol{d}$ and $\delta \tilde{\boldsymbol{p}}$, the linearised weak form 100,102 at iteration $i$ between the configurations of the time interval $\left[t_{n}, t_{n+1}\right]$ leads to the residual vector $\boldsymbol{R}$ with

$$
\left[\begin{array}{ccc}
\boldsymbol{K}_{u u}^{i} & \boldsymbol{K}_{u d}^{i} & \boldsymbol{K}_{u \tilde{p}}^{i} \\
\boldsymbol{K}_{d u}^{i} & \boldsymbol{K}_{d d}^{i}+\boldsymbol{K}_{\varepsilon}^{i} & \boldsymbol{K}_{d \tilde{p}}^{i} \\
\boldsymbol{K}_{\tilde{p} u}^{i} & \boldsymbol{K}_{\tilde{p} d}^{i} & \boldsymbol{K}_{\tilde{p} \tilde{p}}^{i}
\end{array}\right]\left[\begin{array}{c}
\delta \boldsymbol{U} \\
\delta \boldsymbol{d} \\
\delta \tilde{\boldsymbol{p}}
\end{array}\right]=\left[\begin{array}{c}
\boldsymbol{F}_{\mathrm{ext}}-\boldsymbol{F}_{\mathrm{int}}^{i} \\
\boldsymbol{F}_{\psi}^{i}-\boldsymbol{F}_{d}^{i}+\boldsymbol{F}_{\varepsilon}^{i} \\
\boldsymbol{F}_{p}^{i}-\boldsymbol{F}_{\tilde{p}}^{i}
\end{array}\right]=-\boldsymbol{R} .
$$

The force vectors are easily obtained from the right hand sides of the set of Eqs. 100 102, with for the mechanical part

$$
\boldsymbol{F}_{\text {ext }}=\int_{\Omega} \boldsymbol{N}_{u}^{\mathrm{T}} \boldsymbol{f} \mathrm{d} V+\int_{\Gamma_{T}} \boldsymbol{N}_{u}^{\mathrm{T}} \boldsymbol{T} \mathrm{d} S, \text { and } \boldsymbol{F}_{\text {int }}^{i}=\int_{\Omega} \boldsymbol{B}_{u}^{\mathrm{T}} \boldsymbol{\sigma}_{\mathrm{M}}^{i} \mathrm{~d} V,
$$


with for the auxiliary fibre bundle damage part

$$
\begin{array}{r}
\boldsymbol{F}_{\psi}^{i}=-\int_{\Omega} \boldsymbol{N}_{d}^{\mathrm{T}} \frac{l_{\mathrm{I}}}{G_{c}} \psi_{\mathrm{I}, d_{\mathrm{I}}}^{+i} \mathrm{~d} V, \quad \boldsymbol{F}_{d}^{i}=\int_{\Omega}\left(\boldsymbol{N}_{d}^{\mathrm{T}} \boldsymbol{N}_{d}+\boldsymbol{B}_{d}^{\mathrm{T}} \cdot \boldsymbol{c}_{\mathrm{I}} \cdot \boldsymbol{B}_{d}\right) \boldsymbol{d}^{i} \mathrm{~d} V, \text { and } \\
\boldsymbol{F}_{\varepsilon}^{i}=-\int_{\Omega} \boldsymbol{N}_{d}^{\mathrm{T}} \frac{l_{\mathrm{I}}}{G_{c}} \varepsilon\left\langle d_{n+1}^{i}-d_{n}\right\rangle_{-} \mathrm{d} V,(108
\end{array}
$$

and with for the non-local accumulated plastic strain part

$$
\boldsymbol{F}_{p}^{i}=\int_{\Omega} \boldsymbol{N}_{\tilde{p}}^{\mathrm{T}} p_{0}^{i} \mathrm{~d} V, \text { and } \boldsymbol{F}_{\tilde{p}}^{i}=\int_{\Omega}\left(\boldsymbol{N}_{\tilde{p}}^{\mathrm{T}} \boldsymbol{N}_{\tilde{p}}+\boldsymbol{B}_{\tilde{p}}^{\mathrm{T}} \cdot \boldsymbol{c}_{0} \cdot \boldsymbol{B}_{\tilde{p}}\right) \tilde{\boldsymbol{p}}^{i} \mathrm{~d} V .
$$

The stiffness sub-matrices defined in Eq. (106) are obtained from the left hand side of the set of Eqs. 100 102). Starting from Eq. (100) with the linearisation 80 yields the sub-matrices

$$
\begin{aligned}
\boldsymbol{K}_{u u}^{i} & =\int_{\Omega} \boldsymbol{B}_{u}^{\mathrm{T}} \boldsymbol{C}_{\mathrm{M}}^{\varepsilon \varepsilon^{i}} \boldsymbol{B}_{u} \mathrm{~d} V, \\
\boldsymbol{K}_{u d}^{i} & =\int_{\Omega} \boldsymbol{B}_{u}^{\mathrm{T}} \boldsymbol{C}_{\mathrm{M}}^{\varepsilon d^{i}} \boldsymbol{N}_{d} \mathrm{~d} V, \\
\boldsymbol{K}_{u \tilde{p}}^{i} & =\int_{\Omega} \boldsymbol{B}_{u}^{\mathrm{T}} \boldsymbol{C}_{\mathrm{M}}^{\varepsilon \tilde{p}^{i}} \boldsymbol{N}_{\tilde{p}} \mathrm{~d} V,
\end{aligned}
$$

where $\boldsymbol{C}_{\mathrm{M}}^{\varepsilon \varepsilon}$ is the matrix representation of the derivative tensors $\mathbb{C}_{\mathrm{M}}^{\varepsilon \varepsilon} 81, \boldsymbol{C}^{\varepsilon d}$ results from Eq. 82 , and $\boldsymbol{C}_{\mathrm{M}}^{\varepsilon \tilde{p}}$ results from Eq. 83 . The left hand side of Eq. (101) yields the stiffness sub-matrices

$$
\begin{aligned}
\boldsymbol{K}_{d u}^{i} & =-\int_{\Omega} \boldsymbol{N}_{d}^{\mathrm{T}} \boldsymbol{C}_{\mathrm{M}}^{\psi \varepsilon^{i}} \boldsymbol{B}_{u} \mathrm{~d} V, \\
\boldsymbol{K}_{d d}^{i} & =\int_{\Omega}\left[\left(1-C_{\mathrm{M}}^{\psi d^{i}}\right) \boldsymbol{N}_{d}^{\mathrm{T}} \boldsymbol{N}_{d}+\boldsymbol{B}_{d}^{\mathrm{T}} \cdot \boldsymbol{c}_{\mathrm{I}} \cdot \boldsymbol{B}_{d}\right] \mathrm{d} V, \\
\boldsymbol{K}_{\varepsilon}^{i} & =-\int_{\Omega}\left[\frac{l_{\mathrm{I}}}{G_{c}} \varepsilon\left\langle\operatorname{sign}\left(d_{n+1}^{i}-d_{n}\right)\right\rangle_{-}\right] \boldsymbol{N}_{d}^{\mathrm{T}} \boldsymbol{N}_{d} \mathrm{~d} V, \quad \text { and } \\
\boldsymbol{K}_{d \tilde{p}}^{i} & =-\int_{\Omega} C_{\mathrm{M}}^{\psi \tilde{p}^{i}} \boldsymbol{N}_{d}^{\mathrm{T}} \boldsymbol{N}_{\tilde{p}} \mathrm{~d} V,
\end{aligned}
$$

${ }_{541}$ where $\boldsymbol{C}_{\mathrm{M}}^{\psi \varepsilon}$ results from Eq. $84, C_{\mathrm{M}}^{\psi d}$ results from Eq. 85 , and $C_{\mathrm{M}}^{\psi \tilde{p}}$ results from Eq. 86. Finally, using Eqs. 87,89 in Eq. 102 yields the stiffness 
sub-matrices

$$
\begin{aligned}
\boldsymbol{K}_{\tilde{p} u}^{i} & =-\int_{\Omega} \boldsymbol{N}_{\tilde{p}}^{\mathrm{T}} \boldsymbol{C}_{\mathrm{M}}^{p \varepsilon^{i}} \boldsymbol{B}_{u} \mathrm{~d} V, \\
\boldsymbol{K}_{\tilde{p} d}^{i} & =-\int_{\Omega} C_{\mathrm{M}}^{p d^{i}} \boldsymbol{N}_{\tilde{p}}^{\mathrm{T}} \boldsymbol{N}_{d} \mathrm{~d} V, \text { and } \\
\boldsymbol{K}_{\tilde{p} \tilde{p}}^{i} & =\int_{\Omega}\left[\left(1-C_{\mathrm{M}}^{p \tilde{p}^{i}}\right) \boldsymbol{N}_{\tilde{p}}^{\mathrm{T}} \boldsymbol{N}_{\tilde{p}}+\boldsymbol{B}_{\tilde{p}}^{\mathrm{T}} \cdot \boldsymbol{c}_{0} \cdot \boldsymbol{B}_{\tilde{p}}\right] \mathrm{d} V,
\end{aligned}
$$

${ }_{544}$ where $\boldsymbol{C}_{\mathrm{M}}^{p \varepsilon}$ results from Eq. (87), $C_{\mathrm{M}}^{p d}$ from Eq. 88, and $C_{\mathrm{M}}^{p \tilde{p}}$ results from Eq. 89 .

Figure 4 presents the flowchart of the finite element resolution of the phase-field non-local damage multiscale formulation. At the higher scale, the weak form (97, 99) is integrated in time using the finite-element discretisation 103, 105). For each time increment $\left[t_{n}, t_{n+1}\right]$, the solution at configuration $t_{n+1}$ is obtained from the solution at configuration $t_{n}$ through Newton-Raphson iterations using the system (106). In this system, the force vectors $(107,109)$ and the stiffness contributions $(110,119)$ are obtained by assembling the homogenised stress tensor $\boldsymbol{\sigma}_{\mathrm{M}}$ and phases auxiliary equations driving forces $\psi_{\mathrm{I}, d_{\mathrm{I}}}^{+}$and $p_{0}$, and the material tensors $\mathbb{C}_{\mathrm{M}}^{\varepsilon \varepsilon}, \boldsymbol{C}^{\varepsilon d}, \boldsymbol{C}_{\mathrm{M}}^{\varepsilon \tilde{p}}, \boldsymbol{C}_{\mathrm{M}}^{\psi \varepsilon}, C_{\mathrm{M}}^{\psi d}$, $C_{\mathrm{M}}^{\psi \tilde{p}}, C_{\mathrm{M}}^{p \varepsilon}, C_{\mathrm{M}}^{p d}$ and $C_{\mathrm{M}}^{p \tilde{p}}$. These terms arise from the resolution of the $\mathrm{MFH}$ enhanced with damage in both phases as described in Section 3 . Finally, during the MFH resolution, the average stress $\langle\boldsymbol{\sigma}\rangle_{i_{n+1}}$, auxiliary equation driving force, and material operators in the phases $\omega_{i}$ are obtained from the constitutive laws described in Section 2.1 for the inclusion phase and in Section 2.2 for the matrix phase. 


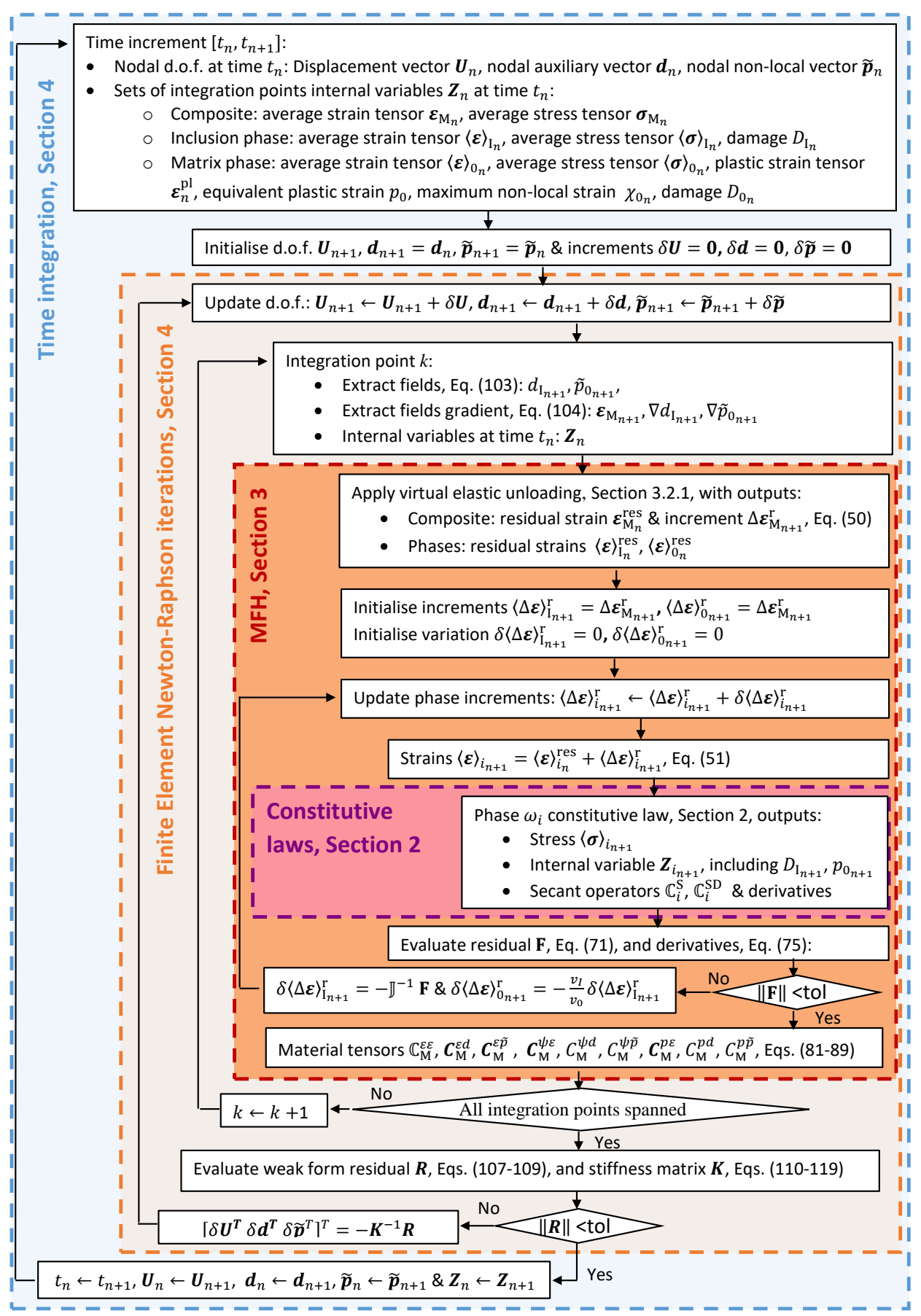

Figure 4: Resolution of the phase-field non-local damage multiscale formulation. 


\section{Identification of material properties and model parameters}

In this section, we first summarise the model parameters and the methods that are used for their identification. We then consider the case of AS4 carbon fibre and 8552 epoxy matrix as a material system. We identify the material parameters of both the fibre bundle and matrix phases from manufacturer data sheets and literature data. The non-local damage parameters are evaluated in order to recover the critical energy release of the matrix material. By considering uni-axial tension tests, we evaluate the phase-field model parameters which allow recovering the right amount of dissipated energy for the failure of a ply loaded along its longitudinal direction.

\subsection{Parameters summary}

Table 1 summarises the properties required by the finite element implementation of the MFH with a damage model embedded in both phases.

First the constituents, both fibre and matrix phases, material behaviours have to be identified. For the fibre, in this work we assume a transverse isotropic behaviour and only the elasticity tensor $\mathbb{C}_{\mathrm{I}}^{\mathrm{el}}, \mathrm{Eq} .(16)$, has to be given. It can be obtained from manufacturer data sheets of micro-mechanical tests performed on the fibres, e.g. [54]. The matrix material properties characterising the linear response, i.e. $\mathbb{C}_{0}^{\mathrm{el}}$ of Eq. (34), and non-linear linear behaviour, i.e. matrix hardening law $\sigma_{Y_{0}}+R_{0}\left(p_{0}\right)$ in Eq. (32) and the damage law evolution $\dot{D}_{0}\left(\varepsilon_{0}, \chi_{0}\right)$, Eq. 35 or 37), can be deduced using the manufacturer elasticity modulus and tensile strength. This allows tuning the hardening and damage evolution laws in order to recover the reported strength, as it will be done here below. However, on the one hand, because the 
Table 1: Model material properties to be identified.

\begin{tabular}{|c|c|c|}
\hline Nature & Property & Method \\
\hline Constituent & Fibre elastic tensor $\mathbb{C}_{\mathrm{I}}^{\mathrm{el}}$, Eq. 16. & $\begin{array}{l}\text { Manufacturer data-sheet } \\
\text { micro-scale experiments. }\end{array}$ \\
\hline Constituent & $\begin{array}{l}\text { Matrix elasticity tensor } \mathbb{C}_{0}^{\mathrm{el}} \text {, Eq. } \\
\text { (34). }\end{array}$ & $\begin{array}{l}\text { Manufacturer data-sheet or resin } \\
\text { experiments. }\end{array}$ \\
\hline Constituent & $\begin{array}{l}\text { Matrix hardening law } \sigma_{Y_{0}}+ \\
R_{0}\left(p_{0}\right) \text {, Eq. }(32) \text {; Damage law } \\
\text { evolution } \dot{D}_{0}\left(\varepsilon_{0}, \chi_{0}\right) \text {, Eq. } \\
\text { or }(37) \text {. }\end{array}$ & $\begin{array}{l}\text { Manufacturer strength and criti- } \\
\text { cal energy release rate, or inverse } \\
\text { analysis from coupons. }\end{array}$ \\
\hline $\begin{array}{l}\text { Embedded } \\
\text { fibre-bundle }\end{array}$ & $\begin{array}{l}\text { Tensile energy release rate } \\
\text { of fibre-bundle breaking and } \\
\text { debonding } G_{c}, \text { Eq. }(30) \text {. }\end{array}$ & Experimental measurements. \\
\hline $\begin{array}{l}\text { Embedded } \\
\text { fibre-bundle }\end{array}$ & $\begin{array}{l}\text { Bundle damage evolution param- } \\
\text { eters } n \text { and } l_{\mathrm{I}} \text {, Eqs. } 77 \text { and } 30 \text {. }\end{array}$ & $\begin{array}{l}\text { From stress build-up profile } \\
(4) \text { and/or uni-axial ply tensile } \\
\text { strength } \sigma_{c} \text {. }\end{array}$ \\
\hline $\begin{array}{l}\text { Matrix crack- } \\
\text { ing direction }\end{array}$ & $\begin{array}{l}\text { Matrix squared lengths tensor } \\
\boldsymbol{c}_{0}, \text { Eq. } 38 \text {. }\end{array}$ & $\begin{array}{l}\text { From transverse critical energy } \\
\text { release rate \& Constrained ma- } \\
\text { trix cracking direction. }\end{array}$ \\
\hline
\end{tabular}

matrix non-linear behaviour changes in a composite as compared to its neat bulk behaviour it is possible to use an inverse analysis from composite coupon experiments [36], and, on the other hand, rigorously the model parameters should satisfy both matrix strength and critical energy release rate and this requires to identify the transverse non-local lengths, i.e. $\sqrt{c_{0_{1}}}=\sqrt{c_{0_{2}}}$ defining the matrix squared lengths tensor $\boldsymbol{c}_{0}$, Eq. (38), and the damage model 
altogether 36.

The critical energy $G_{c}$, Eq. (30), related to the embedded fibre bundle tensile breaking and debonding can be measured from Compact Tension Specimen [55] or Double Edge Notched Test specimen [56]. Indeed, when the fibre bundles are embedded in a matrix, the fracture of fibre is accompanied with fibre/matrix interface debonding, matrix micro-cracking, and finally by the final fibre pull-out from the matrix. Therefore, a much higher energy is dissipated during the fibre breaking process in composites than that of neat fibre breaking and should thus be measured accordingly. The embedded fibre bundle damage evolution is defined by the two damage evolution parameters $n$ and $l_{\mathrm{I}}$, Eqs. (7) and (30). As discussed here below, a relation between them can be derived from the stress build-up profile, see Fig. 1 and Eq. (4), whilst a second relation results from the uni-axial ply tensile strength $\sigma_{c}$, which can be experimentally measured or is given by the manufacturer data sheets.

Finally, the matrix squared lengths tensor $\boldsymbol{c}_{0}$, Eq. (38), is defined in order to represent the anisotropic nature of the matrix cracking in a UD ply. Whilst the transverse characteristic lengths $\sqrt{c_{0_{1}}}=\sqrt{c_{0_{2}}}$ can be chosen in order to recover the critical energy release rate of transverse failure [36], see Appendix $\mathrm{C}$, the third characteristic length $\sqrt{c_{0_{3}}}$ is taken large enough to constrain matrix cracking along the fibre direction.

\subsection{Case of $A S_{4}$ carbon fibre and 8552 epoxy matrix}

\subsubsection{Phases material properties}

The studied composite material is a UD-carbon fibre reinforced epoxy. The AS4 carbon fibre and 8552 epoxy components are used as reference 
materials and their mechanical properties are collected from product data sheet of Hexcel [57, 58] and completed with data from the literature [4, 54, 59].

Table 2: Material properties of the embedded AS4 carbon fibres.

\begin{tabular}{ll}
\hline Property & Value \\
\hline Long. Young's modulus $E_{\mathrm{I}}^{3}[\mathrm{GPa}]$ & $231.0[54]$ \\
Trans. Young's modulus $E_{\mathrm{I}}^{1}[\mathrm{GPa}]$ & $12.99[54]$ \\
Trans. Poisson's ratio $\nu_{\mathrm{I}}^{12}[-]$ & $0.46[54]$ \\
Long.-Trans. Poisson's ratio $\nu_{\mathrm{I}}^{31}[-]$ & $0.3[54]$ \\
Trans. shear modulus $\mu_{\mathrm{I}}^{12}[\mathrm{GPa}]$ & $4.45[54]$ \\
Long.-Trans. shear modulus $\mu_{\mathrm{I}}^{31}[\mathrm{GPa}]$ & $11.3[54]$ \\
\hline Tensile Strength $X_{\mathrm{I}}^{\mathrm{t}}[\mathrm{MPa}]$ & $4413[58]$ \\
Carbon fibre radius $r[\mu \mathrm{m}]$ & $3.55[58]$ \\
Energy release rate of fibres $G_{c_{\mathrm{I}}}\left[\mathrm{J} / \mathrm{m}^{2}\right]$ & $52[54]$ \\
\hline
\end{tabular}

Carbon fibre bundles. The phase-field model of the fibre bundle material phase was presented in Section 2.1. The continuous PAN based carbon fibres AS4 are modelled using a transverse isotropic linear elastic constitutive model, see Eq. (16). The typical mechanical elastic properties of PAN based high strain carbon fibres are presented in Table 2.

When it comes to the properties related to the tensile failure, the measured critical energy release rate was $G_{c_{\mathrm{I}}}=52 \mathrm{~N} / \mathrm{m}$ for AS4 fibre in reference [54. However, as said, when the fibre bundles are embedded in a matrix, the energy dissipated during the fibre breaking process in composites is not the one of neat fibre breaking and a higher critical energy release rate was reported in 4 for fibres of a composite ply and is used in this work, see Table 5. 
Epoxy matrix. The non-local damage model was presented in Section 2.2.1.

It is assumed that the epoxy matrix follows an elasto-plastic behaviour model and its hardening law defining the yield surface $(32)$ reads

$$
R_{0}\left(p_{0}\right)=h_{0}\left(1-\exp \left(-m_{0} p_{0}\right)\right)
$$

where $p_{0}$ is the accumulated plastic strain of the material, and where $h_{0}$ and $m_{0}$ are the material parameters. Furthermore, either a Lemaitre [42] scalar damage model (35) or a saturated damage law (37) can be adopted.

The elastic properties of the cured 8552 epoxy are taken from the manufacturer data sheet. By lack of elasto-plastic data, the approximated elastoplastic and damage parameters are adopted to match the tensile strength $X_{0}^{\mathrm{t}}$ of $121 \mathrm{MPa}$ reported for 8552 epoxy, for both damage models. All the necessary material parameters are reported in Table 3 , in which the characteristic length of the non-local model is evaluated in order to recover the failure critical energy release rate of the bulk matrix $G_{c_{0}}$, see Appendix C. This length actually depends on the damage model used. Besides, when using the matrix model in the damage enhanced $\mathrm{MFH}$, the non-local lengths have to be reevaluated, on the one hand, in order to recover the transverse intra-laminar failure critical energy release rate $G_{c T}$, see Appendix C, with the final values reported in Table 5, and, on the other hand, in order to have an anisotropic behaviour with the length along the fibres being larger.

\subsubsection{Determination of phase-field parameters of the fibre bundle phase}

In this section, the two parameters $n$ and $l_{\text {I }}$ of phase-field model used in Eqs. (7) and (30) are determined under two constraints arising from the mechanical properties of fibre and matrix. 
Table 3: Material properties of the matrix.

\begin{tabular}{ll}
\hline Property & Value \\
\hline Young's modulus $E_{0}[\mathrm{GPa}]$ & $4.668[57]$ \\
Poisson's ratio $\nu_{0}[-]$ & 0.39 \\
Initial yield stress $\sigma_{\mathrm{Y} 0}[\mathrm{MPa}]$ & 32.0 \\
\hline Hardening modulus $h_{0}[\mathrm{MPa}]$ & 300.0 \\
Hardening exponent $m_{0}[-]$ & 100.0 \\
\hline Bulk matrix Tensile strength $X_{0}^{\mathrm{t}}[\mathrm{MPa}]$ & $121[57]$ \\
Bulk matrix critical energy release rate of $G_{c_{0}}\left[\mathrm{~J} / \mathrm{m}^{2}\right]$ & $\simeq 100[59]$ \\
\hline Lemaitre damage critical energy release $S_{0}[\mathrm{MPa}]$ & 0.21 \\
Lemaitre damage exponent $s_{0}[-]$ & 2.0 \\
Lemaitre damage critical plastic strain $p_{\mathrm{C} 0}[-]$ & 0.0 \\
Characteristic lengths bulk matrix with Lemaitre model & $30 \times 10^{-3}$ \\
$\sqrt{c_{0_{1}}}=\sqrt{c_{0_{2}}}=\sqrt{c_{0_{3}}}[\mathrm{~mm}]$ & \\
\hline Saturated damage threshold $D_{\text {max }}[-]$ & 0.99 \\
Saturated damage exponent $s_{0}[-]$ & 700 \\
Saturated damage plastic strain threshold $p_{\mathrm{C} 0}[-]$ & 0.007 \\
Characteristic lengths bulk matrix with saturated model & $20 \times 10^{-3}$ \\
$\sqrt{c_{0_{1}}}=\sqrt{c_{0_{2}}}=\sqrt{c_{0_{3}}}[\mathrm{~mm}]$ & \\
\hline
\end{tabular}

The first constraint is determined based on the limited maximum shear stress $\tau_{\max }$ that arises in the stress build-up profile (4) at the fibre-matrix interface of embedded broken fibres. Since the shear stress at the fibre-matrix interface reads $\tau=\frac{r}{2} \frac{\partial \sigma}{\partial x}$, where $r$ is the radius of a fibre, its maximum value can be computed through Eq. (4) and is expressed as

$$
\tau_{\max }=\frac{n r \sigma_{\infty}}{2 l_{\mathrm{I}}} \times \max _{x \in R}\left[\left(1-\exp \left(-\frac{|x|}{l_{\mathrm{I}}}\right)\right)^{n-1} \exp \left(-\frac{|x|}{l_{\mathrm{I}}}\right)\right] .
$$


The measurement of [1] shows that the maximum shear stress $\tau_{\max }$ at the fibre-matrix interface is approximately equal to the yielding stress, $\sigma_{\mathrm{Y} 0}$, of the matrix. Assuming that the tensile strength $X_{\mathrm{I}}^{\mathrm{t}}$ of carbon fibre can be used as $\sigma_{\infty}$ at failure point, and using the properties of Table 2, the parameters in Eq. 121 are summarised as follows

$$
\tau_{\max }=32.0 \mathrm{MPa}, \quad \sigma_{\infty}=4413 \mathrm{MPa} \quad \text { and } \quad r=3.55 \mu \mathrm{m} .
$$

Equation (121), together with the parameters reported in Eq. (122), provides a first constraint between $n$ and $l_{\mathrm{I}}$.

The second constraint results from the tensile strength of the composite material: the longitudinal tensile strength of the composite material predicted by the MFH scheme embedding the phase-field fibre damage model needs to match the reported experimental values.

Uni-axial tensile test on fibre bundle with uniform damage solution. The phase-field damage model of a fibre under uni-axial tension along the longitudinal direction presented in Section 2.1 reads

$$
\begin{aligned}
\sigma & =E_{\mathrm{I}}^{3 \mathrm{D}} \varepsilon, \text { and } \\
d_{\mathrm{I}}-l_{\mathrm{I}} \nabla^{2} d_{\mathrm{I}} & =-\frac{l_{\mathrm{I}}}{2 G_{c}} \frac{\partial}{\partial d_{\mathrm{I}}}\left[\left(1-d_{\mathrm{I}}\right)^{n} E_{\mathrm{I}}^{3}\right] \varepsilon^{2} .
\end{aligned}
$$

The maximum value of stress, $\sigma$, can be obtained easily by solving the set of Eqs. (123) and (124) under a uniformity assumption, i.e. $\nabla^{2} d_{\mathrm{I}}=0$, yielding

$$
d_{\mathrm{I}}=\frac{n\left(1-d_{\mathrm{I}}\right)^{n-1} l_{\mathrm{I}}}{2 G_{c}} E_{\mathrm{I}}^{3} \varepsilon^{2}
$$

First, according to the experimental measurements in [1], it has been shown in [34] that the shape parameter $n \in[2,3]$ can be used to describe 
Table 4: Parameter $n$ and its corresponding $l_{\mathrm{I}}$ according to Eq. (121).

\begin{tabular}{c|cccccc}
\hline$n$ & 2.0 & 2.1 & 2.2 & 2.3 & 2.4 & 2.5 \\
$l_{\mathrm{I}}[\mu \mathrm{m}]$ & 122.4 & 120.2 & 118.3 & 116.6 & 115.1 & 113.8 \\
\hline$n$ & 2.6 & 2.7 & 2.8 & 2.9 & 3.0 & \\
$l_{\mathrm{I}}[\mu \mathrm{m}]$ & 112.6 & 111.5 & 110.5 & 109.6 & 108.8 & \\
\hline
\end{tabular}

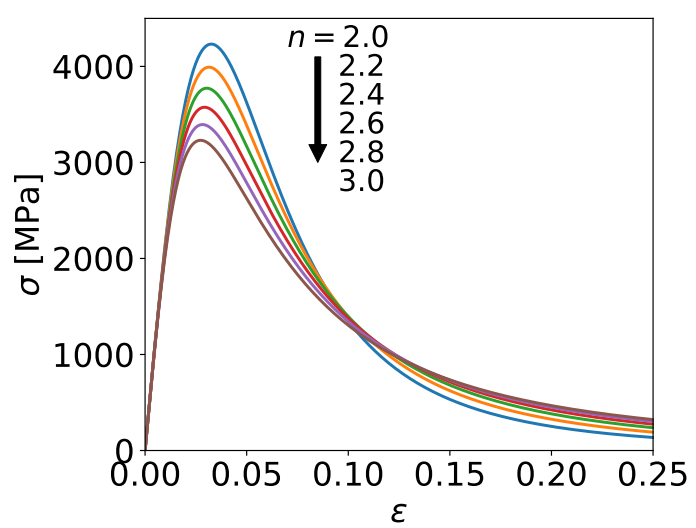

Figure 5: The strain-stress curves for different values of $n$ of the longitudinal tensile case for fibre with uniform damage.

the stress build-up profile (4) of embedded broken fibres. For given values of $n \in[2,3], l_{\mathrm{I}}$ can be computed by solving Eq. (121). The resulting values of $l_{\mathrm{I}}$ are listed in Table 4 in terms of the corresponding assumptions on the value of $n$. Submitting the couples $n$ and $l_{\mathrm{I}}$ to Eq. (125), and letting $d$ increase from 0 to 1, the strain $\varepsilon$ and stress $\sigma$ can be computed successively with Eqs. 123 and (125). Using the values of $E_{\mathrm{I}}^{3}=231.0 \mathrm{GPa}$ and $G_{c}=90.0 \mathrm{~N} / \mathrm{mm}$ reported in Table 2 and in Table 5, the strain-stress curves of the uniform damage $1 \mathrm{D}$ cases are presented in Fig. 5 for different values of $n \in[2,3]$.

Since the longitudinal tensile strength $\sigma_{\mathrm{c}}$ of UD fibre reinforced composite is dominated by the fibre failure, for a reported composite tensile strength 
674

of $\sigma_{\mathrm{c}}=2205.0 \mathrm{MPa}$ for a fibre volume fraction $v_{\mathrm{I}}=60 \%$ [58], the expected maximum tensile stress of fibre at composite failure is around $\frac{\sigma_{\mathrm{c}}}{v_{\mathrm{I}}}=3675.0$ MPa. According to the strain-stress curves presented in Fig. 5, the value of the parameter $n$ will be above 2.4 , and the corresponding length $l_{\mathrm{I}}$ is readily deduced from Table 4

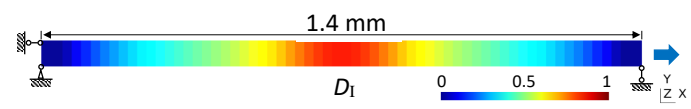

(a) Fibre damage at loading stage $1 ; \sqrt{c_{0_{3}}}=$ $l_{\mathrm{I}}$

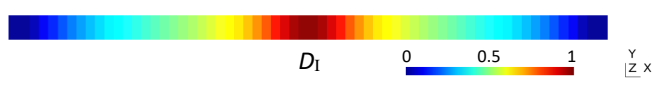

(c) Fibre damage at loading stage $2 ; \sqrt{c_{0_{3}}}=$ $l_{\mathrm{I}}$

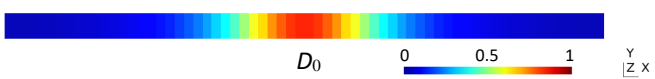

(e) Matrix damage at loading stage 1 ; $\sqrt{c_{0_{3}}}=l_{\mathrm{I}}$
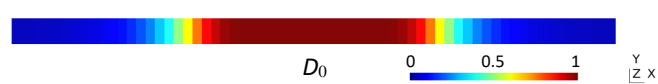

(g) Matrix damage at loading stage 2; $\sqrt{c_{0_{3}}}=l_{\mathrm{I}}$

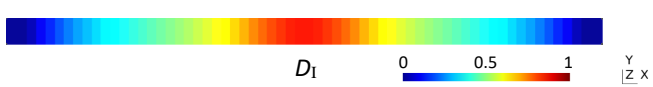

(b) Fibre damage at loading stage $1 ; \sqrt{c_{0_{3}}}=$ $\sqrt{2} \mathrm{~mm}$

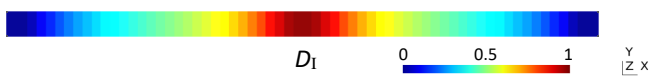

(d) Fibre damage at loading stage $2 ; \sqrt{c_{0_{3}}}=$ $\sqrt{2} \mathrm{~mm}$

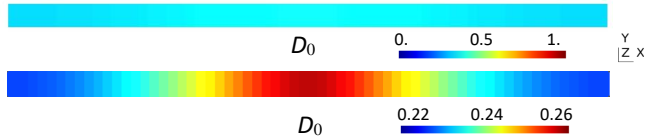

(f) Matrix damage at loading stage 1; $\sqrt{c_{0_{3}}}=\sqrt{2} \mathrm{~mm}$

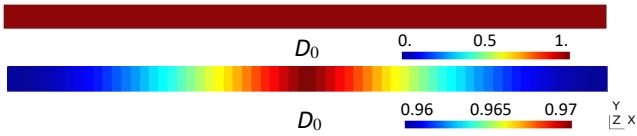

(h) Matrix damage at loading stage 2; $\sqrt{c_{0_{3}}}=\sqrt{2} \mathrm{~mm}$

Figure 6: Schematics of the uni axial composite material loading and damage distributions of fibre and matrix phases at two different loading stages marked with crosses on the strain-stress curve in Fig. 7 (b) for $\sqrt{c_{0_{3}}}=l_{\mathrm{I}}$ (left column) and for $\sqrt{c_{0_{3}}}=\sqrt{2} \mathrm{~mm}$ (right column). 
Uni-axial tensile test on composites. The developed MFH multiscale method presented in Section 3 and implemented using the finite element method in Section 4 is applied to a uni-axial tensile test of a $2 \mathrm{D}$ composite sample under plane strain condition with an element size $l_{\text {element }} \approx l_{\mathrm{I}} / 5$. The damage initiation in the centre is enforced through the application of a Dirichlet boundary condition $d_{\mathrm{I}}=0$ applied at the left and right edges of the sample, see the schematics in Fig. 6(a), Applying this boundary condition requires a specimen length such that both left and right edges are more than $6 \times l_{\text {I }}$ away from the damaging centre. Therefore, a sample length of $1.4 \mathrm{~mm}$ is used according to the value of $l_{\mathrm{I}}$ reported in Table 4, whilst the width is set to $0.06 \mathrm{~mm}$. We consider the composite material with a fibre volume fraction, $v_{\mathrm{I}}$, of $60 \%$. The required material properties are listed in Tables 2 and 3 .

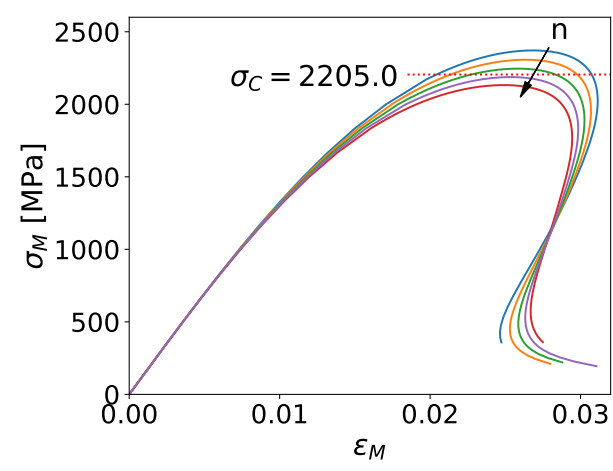

(a) $\sqrt{c_{0_{3}}}=l_{\mathrm{I}}$

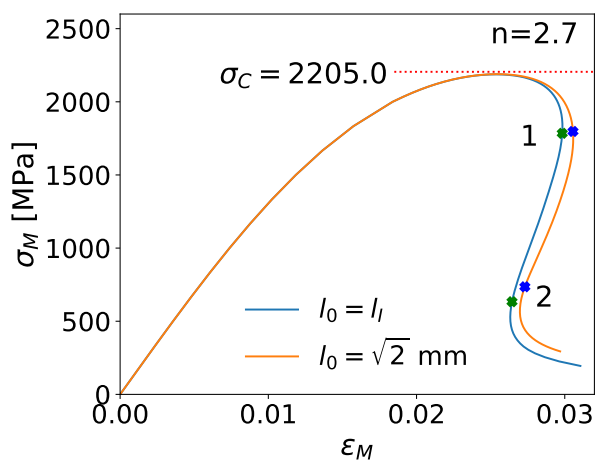

(b) $n=2.7$

Figure 7: The strain-stress curves of the longitudinal tensile test of the 2D composite sample: (a) For $\sqrt{c_{0_{3}}}=l_{\mathrm{I}}$ and for successively $n=2.4,2.5,2.6,2.7,2.8$; the arrow indicates the increasing direction of $n$; and (b) For $n=2.7$ and for successively $\sqrt{c_{0_{3}}}=l_{\mathrm{I}}$ in blue and $\sqrt{2} \mathrm{~mm}$ in orange. 
First, the characteristic length along the longitudinal direction of the fibres for the matrix non-local damage model is set to be $\sqrt{c_{0_{3}}}=l_{\mathrm{I}}$, see Table 4. The global strain-stress evolution of the $2 \mathrm{D}$ tensile sample is successively evaluated for $n=2.4,2.5,2.6,2.7,2.8$ using a path following analysis in order to capture the snapback behaviours. For the studied material system, which has a reported longitudinal tensile strength of 2205.0 MPa [58], the values of $n$ and $l_{\mathrm{I}}$ can be determined according to the strain-stress curves reported in Fig. 7(a), which indicates that the value of $n$ should be slightly lower than 2.7. Eventually, the values of

$$
n=2.7, \sqrt{c_{0_{3}}}=l_{\mathrm{I}} \quad \text { and } \quad l_{\mathrm{I}}=111.5 \mu \mathrm{m},
$$

are adopted in the following applications unless otherwise stated.

Considering $n=2.7$, the effect of the characteristic length for the matrix non-local damage model is studied on the 2D tensile test using successively $\sqrt{c_{0_{3}}}=l_{\mathrm{I}}$ and $\sqrt{c_{0_{3}}}=\sqrt{2} \mathrm{~mm}$. In Fig. $7(\mathrm{~b})$, it can be seen that changing the characteristic length of the matrix non-local damage model has no effect on the maximum stress of the tensile sample. However, a longer non-local damage length $\sqrt{c_{0_{3}}}$ leads to slightly more energy dissipation since the snapback is slightly less pronounced. The higher energy dissipation resulting from a longer $\sqrt{c_{0_{3}}}$ can be explained easily by the size of the matrix damage zone as shown in Fig. 6, which presents the damage zones of both fibre and matrix phases at the two different loading stages marked with crosses on the strainstress curves in Fig. 7(b), successively for $\sqrt{c_{0_{3}}}=l_{\mathrm{I}}$ and $\sqrt{2} \mathrm{~mm}$. The fibre damage zone reflects the number of broken fibres in the fibre bundles: Figs. 6(a) and 6(c) for $\sqrt{c_{0_{3}}}=l_{\mathrm{I}}$, and Figs. 6(b) and 6(d) for $\sqrt{c_{0_{3}}}=\sqrt{2} \mathrm{~mm}$, show this evolution from the points in which half of the fibres are broken, 
up to the final stage in which the full fibre bundle is broken. The damage of the matrix phase reflects the cracking of matrix and the debonding at fibrematrix interface. The matrix damage in Figs. $6(\mathrm{e})$ and $6(\mathrm{~g})$ for $l_{30}=l_{\mathrm{I}}$, and in Figs. 6(f) and 6(h) for $\sqrt{c_{0_{3}}}=\sqrt{2} \mathrm{~mm}$, represents the evolution from the matrix cracking and fibre-matrix debonding around the fibre breaking point up to the final fibre pull-out stage. When comparing Figs. 6(a)- 6(d), the fibre damage zones do not show any difference for $\sqrt{c_{0_{3}}}=l_{\mathrm{I}}$ and $\sqrt{c_{0_{3}}}=\sqrt{2}$ $\mathrm{mm}$. This indicates that the matrix damage has no effect on the fibre damaging process for a uni-axial tension and that the failure is dominated by the fibres. When comparing Figs. 6(e), 6(h), the matrix damage concentrates in the centre of the sample for $\sqrt{c_{0_{3}}}=l_{\mathrm{I}}$, whilst it propagates throughout the sample for $\sqrt{c_{0_{3}}}=\sqrt{2} \mathrm{~mm}$, explaining the higher ductility of this last case.

Table 5: Material properties related to the composite material failure modelled using MFH.

Transverse critical energy release rate of $G_{c T} \simeq \quad \simeq 100 \mathrm{~J} / \mathrm{m}^{2}[59]$

Characteristic lengths in MFH with Lemaitre model

$$
\begin{array}{ll}
\sqrt{c_{0_{1}}}=\sqrt{c_{0_{2}}}[\mathrm{~mm}] & 110 \times 10^{-3} \\
\sqrt{c_{0_{3}}}[\mathrm{~mm}] & \sqrt{2}
\end{array}
$$

Characteristic lengths in MFH with saturated model

\begin{tabular}{ll}
$\qquad \sqrt{c_{0_{1}}}=\sqrt{c_{0_{2}}}[\mathrm{~mm}]$ & $50 \times 10^{-3}$ \\
$\sqrt{c_{0_{3}}}[\mathrm{~mm}]$ & $\sqrt{2}$ \\
\hline Tensile critical energy release rate $G_{c}[\mathrm{~N} / \mathrm{mm}]$ & $90.0[4]$ \\
Longitudinal strength $\sigma_{\mathrm{c}}[\mathrm{MPa}]$ for $v_{\mathrm{I}}=60 \%$ & $2205[58]$ \\
\hline Phase-field length $l_{\mathrm{I}}[\mathrm{mm}]$ & 0.111 \\
Phase-field exponent $n[-]$ & 2.7
\end{tabular}




\section{Applications}

The developed MFH embedding a non-local damage approach for the matrix phase and a phase-field approach for the fibre bundle phase is now applied to study the failure of a notched laminate and the failure of a plain woven composite unit-cell.

\subsection{Applications on a notched laminate}

The failure of a notched laminate was studied with a MFH method embedding a local approach of fibre bundle damage in [34]. Because of the local formalism the simulation exhibited a lack of convergence when some finite elements were reaching local softening because of the fibre bundle damaging process. In this section we show that the phase-field approach, on the one hand, allows conducting the simulation to an end, and, on the other hand, predicts the failure modes in good agreement with the experimental Computed Tomography (CT) observations reported in the literature [3].

\subsubsection{Geometry}

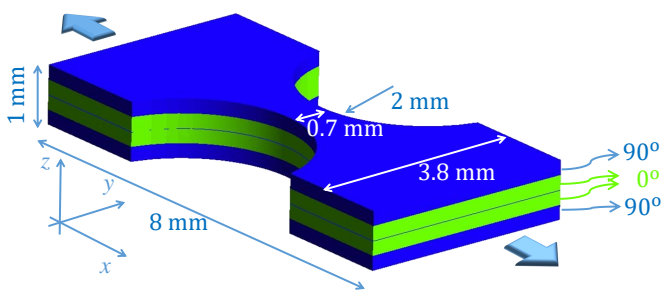

(a)

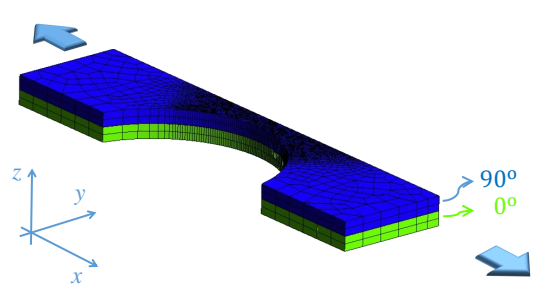

(b)

Figure 8: Double notched sample laminate redrawn from [34]: (a) Geometry and stacking sequence of the sample; and (b) Finite element discretisation of one quarter of the notched sample. 
A double notched sample extracted from a UD laminate is illustrated in Fig. 8(a), The layup corresponds to a $\left[90^{\circ} / 0^{\circ}\right]_{S}$ stacking sequence. One quarter of the sample is discretised into finite elements as illustrated in Fig. 8(b), Quadratic hexahedral elements are considered, and the element size at the notched part is about $40 \mu \mathrm{m}$ in the $x-y$ plane, so that the distance between integration points remains lower than the matrix non-local and phase-field characteristic lengths.

A tensile test is studied using a dynamic implicit solver.

\subsubsection{Material properties}

The exact matrix and fibre material system was not provided in Ref. [3]. We thus consider a composite material made of the 8552 epoxy resin, modelled with a saturated damage law and whose properties are reported in Table 3, reinforced with AS4 fibre, whose properties are reported in Table 2. We consider a nominal fibre volume fraction $v_{\mathrm{I}}=0.6$ for the AS4/8552 UD composite material which is modelled using the MFH approach embedded with a non-local damage approach for the matrix and a phase-field approach for the fibre bundle damaging process as presented in Section 3 . The phasefield and non-local damage auxiliary equations parameters are reported in Table 5. Quadratic hexahedral elements were used in this simulations with linear shape functions for the auxiliary equations.

The inter-laminar failure is governed by a delamination law. As discussed in [34, delamination initiation is triggered by the criterion

$$
\frac{\ll \sigma \gg^{2}}{\hat{\sigma}_{\text {IC }}^{2}}+\frac{\tau^{2}}{\hat{\tau}_{\text {IIC }}^{2}} \leq\left(1-D_{0}\right)^{2},
$$

where $\hat{\sigma}_{\text {IC }}$ and $\hat{\tau}_{\text {IIC }}$ are the maximum tension and shearing of the cohesive 
model. The presence of the matrix damage $D_{0}$ in Eq. (127) accounts for the existence of the damaging process taking place in the plies. The delamination process is governed by the two delamination modes energy release rates $G_{\mathrm{I}}$ and $G_{\mathrm{II}}$, with a complete fracture obtained for

$$
\left(\frac{G_{\mathrm{I}}}{G_{\mathrm{IC}}}\right)^{\alpha}+\left(\frac{G_{\mathrm{II}}}{G_{\mathrm{IIC}}}\right)^{\alpha}=1,
$$

where $G_{\mathrm{IC}}$ and $G_{\mathrm{IIC}}$ are the mode I and mode II critical energy release rates respectively, and where $\alpha$ is a mixed mode parameter. The surface traction is governed by an effective stress $\sigma_{\text {eff }}$ which obeys to an exponential law in terms of the maximum reached opening $\Delta_{\max }=\max _{t^{\prime} \leq t}\left(\Delta\left(t^{\prime}\right)\right)$ during the delamination process as detailed in [34]. The delamination model parameters listed in Table 6 were used in [34] although they correspond to values used for IM7/8552 carbon-epoxy composite laminates in Ref. [4], with a critical stress reduced to $25[\mathrm{MPa}]$ to account for the finite size of the elements.

Table 6: Material properties of the delamination model [34.

\begin{tabular}{ll}
\hline Property & Value \\
\hline Mode I critical energy release rate $G_{\mathrm{IC}}\left[\mathrm{J} / \mathrm{m}^{2}\right]$ & 277.0 \\
Mode II critical energy release rate $G_{\mathrm{IIC}}\left[\mathrm{J} / \mathrm{m}^{2}\right]$ & 788.0 \\
Mode I critical stress $\hat{\sigma}_{\mathrm{IC}}[\mathrm{MPa}]$ & 25 \\
Mode II critical stress $\hat{\tau}_{\mathrm{II}}[\mathrm{MPa}]$ & 25 \\
Mixed mode parameter $\alpha[-]$ & 1.0 \\
\hline
\end{tabular}



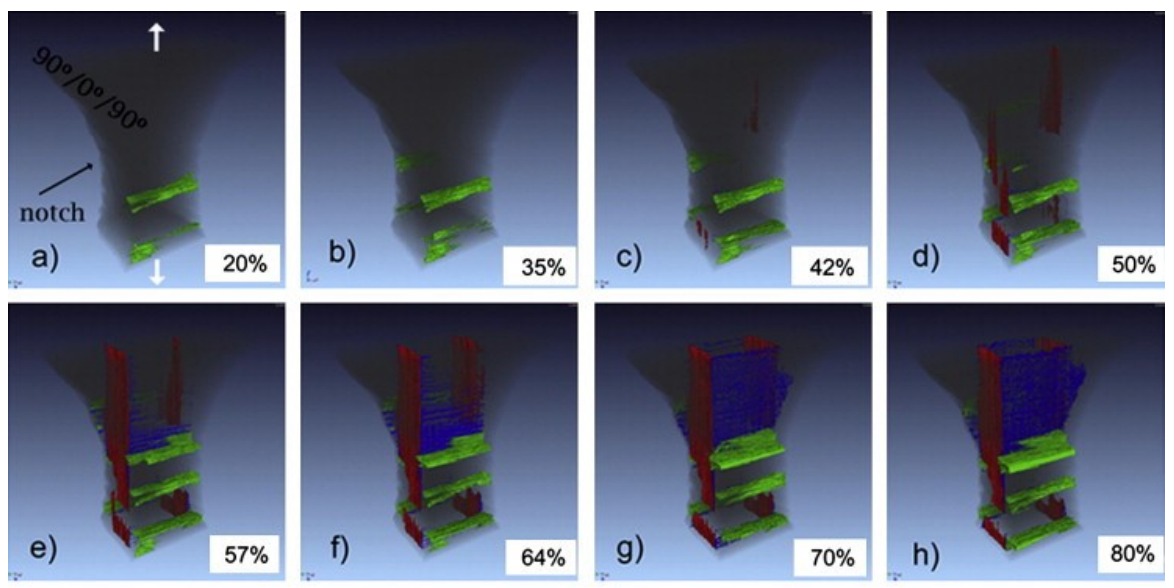

Transverse Ply Cracks

$0^{\circ}$ Splits

Delaminations

$\%$ of final failure load

Figure 9: Experimental damage modes of the notched sample as observed in Ref. 3]. Reprinted from Composites Science and Technology, 71/12, A.E. Scott and M. Mavrogordato and P. Wright and I. Sinclair and S.M. Spearing, In situ fibre fracture measurement in carbonepoxy laminates using high resolution computed tomography, 1471-1477, Copyright (2011), with permission from Elsevier.

\subsubsection{Results}

A double notched sample of the same geometry was in situ tested so that the damage modes could be observed by Synchrotron radiation Computed Tomography (CT) in Ref. [3]. The different damage modes experimentally observed are illustrated in Fig. 9.

Figure 10 compares the forces vs. displacement curves obtained by considering successively a local damage model 34] and a phase-field damage model for the fibre bundles. Whilst the local approach fails when the damage localises in a finite element, preventing the simulation to be achieved, the phase-field method proceeds up to failure of the laminate. The damage and delamination distributions predicted for the four configurations indicated in 


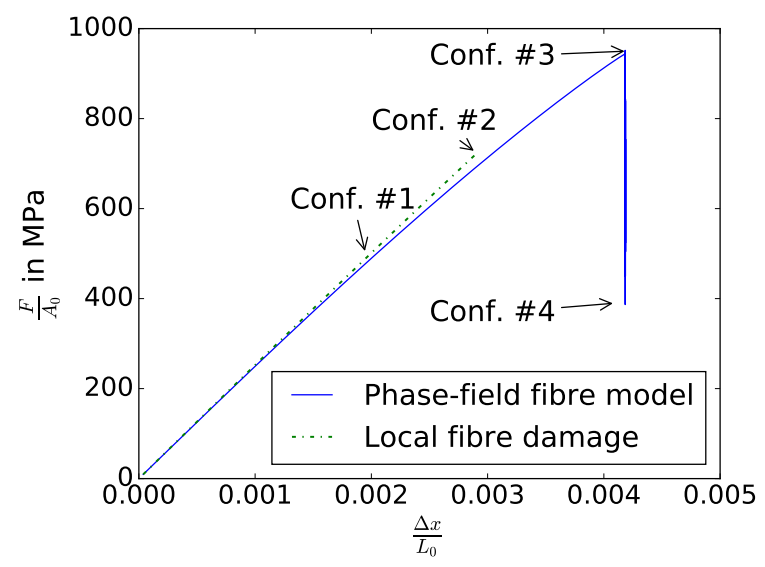

Figure 10: Comparison between numerical predictions of the MFH framework using either a local damage model 34 or a phase-field formulation to represent the failure of the fibre bundle phase.

Fig 10 are reported in Figs. 11,13.

For a load corresponding to about $50 \%$ of the maximum load, i.e. at configuration \#1, the damage and delamination distributions obtained by the two approaches are comparable, see Fig. 11, except concerning the fibre bundle damage in the $0^{\circ}$-ply which concentrates at the notch with the local approach, see Fig. 11(e), The damage distributions can also be compared to the experimental observations of Fig. 9(d). For the $0^{\circ}$-ply, the damage evolution in the matrix, see Figs. 11(a) 11(b) forms the so-called $0^{\circ}$ splits, which are experimentally observed in Fig. 9(d). For the $90^{\circ}$-ply, the damage develops only in the matrix near the notch, see Figs. 11(c) [11(d), in agreement with Fig. 9(d). The slight delamination predicted at the notch in Figs. $11(\mathrm{~g})$ 11(h) is visible on the CT-scan image related to the $57 \%$ loading, see Fig. 9(e).

For a load corresponding to about $70 \%$ of the maximum load, i.e. at con- 


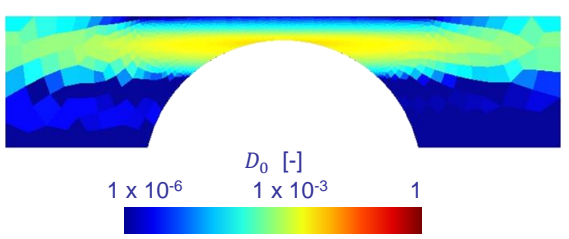

(a) $0^{\circ}$-ply-Local

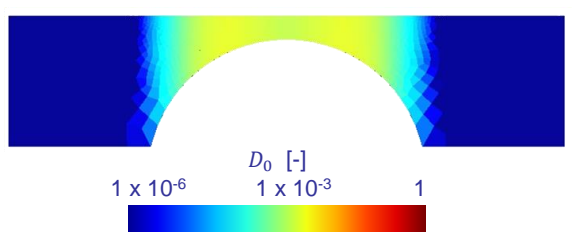

(c) $90^{\circ}$-ply-Local

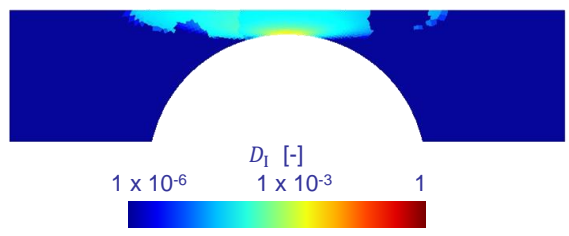

(e) $0^{\circ}$-ply-Local

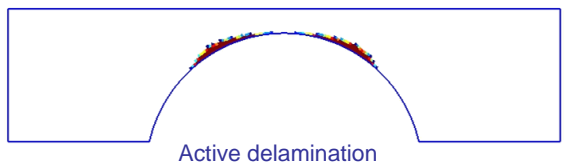

(g) $0^{\circ} / 90^{\circ}$-interface-Local

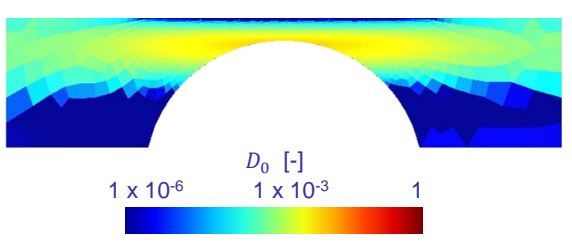

(b) $0^{\circ}$-ply-Phase-Field

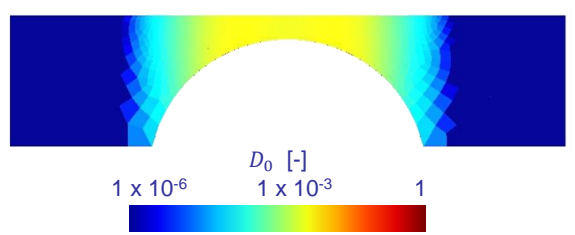

(d) $90^{\circ}$-ply-Phase-field

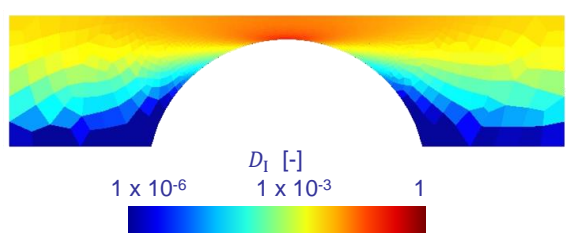

(f) $0^{\circ}$-ply-Phase-field

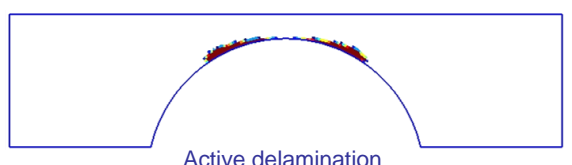

(h) $0^{\circ} / 90^{\circ}$-interface-Phase-Field

Figure 11: Damage and delamination distributions for the notched sample at configuration \#1, see Fig. 10. predicted with the local damage formulation (left column) and the phasefield formulation (right column) of the fibre bundle damage process: (a-b) Matrix damage (logarithmic scale) in the $0^{\circ}$ ply; (c-d) Matrix damage (logarithmic scale) in the $90^{\circ}$ ply; (e-f) Fibre bundle damage (logarithmic scale) in the $0^{\circ}$ ply; and (g-h) Delamination zones at the $0^{\circ}-90^{\circ}$ interface. 


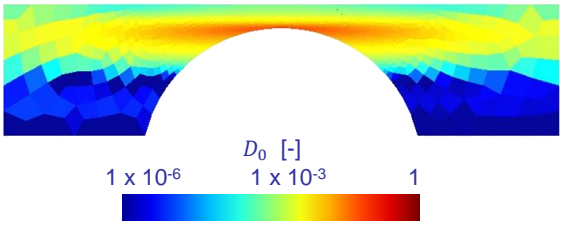

(a) $0^{\circ}$-ply-Local

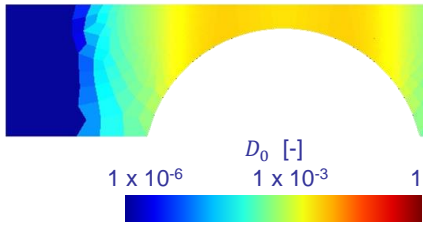

(c) $90^{\circ}$-ply-Local

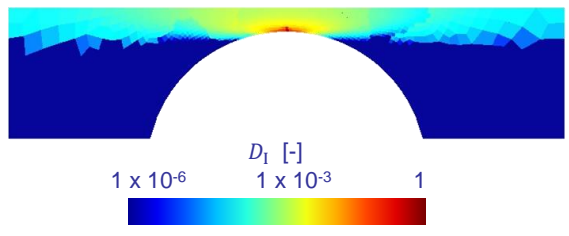

(e) $0^{\circ}$-ply-Local

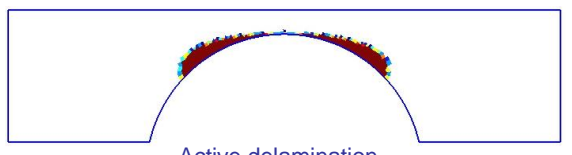

Active delamination

(g) $0^{\circ} / 90^{\circ}$-interface-Local

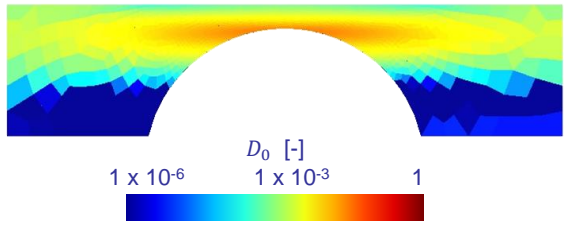

(b) $0^{\circ}$-ply-Phase-Field
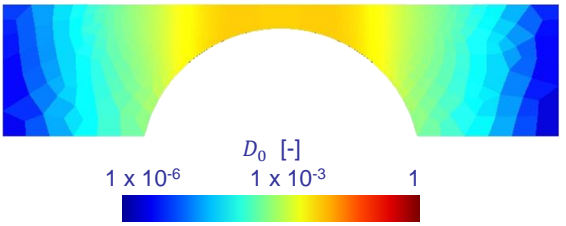

(d) $90^{\circ}$-ply-Phase-field

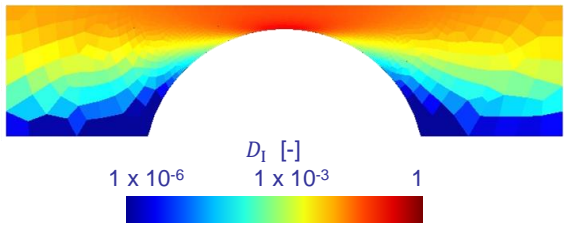

(f) $0^{\circ}$-ply-Phase-field

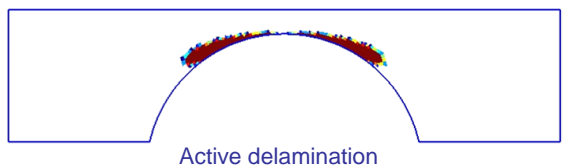

(h) $0^{\circ} / 90^{\circ}$-interface-Phase-Field

Figure 12: Damage and delamination distributions for the notched sample at configuration \#2, see Fig. 10 predicted with the local damage formulation (left column) and the phasefield formulation (right column) of the fibre bundle damage process: (a-b) Matrix damage (logarithmic scale) in the $0^{\circ}$ ply; (c-d) Matrix damage (logarithmic scale) in the $90^{\circ}$ ply; (e-f) Fibre bundle damage (logarithmic scale) in the $0^{\circ}$ ply; and (g-h) Delamination zones at the $0^{\circ}-90^{\circ}$ interface. 


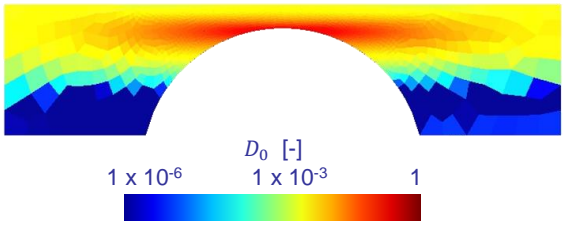

(a) $0^{\circ}$-ply-Phase-Field, \#3

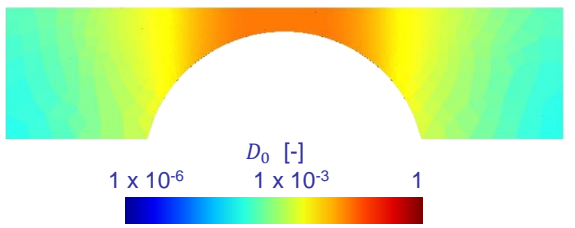

(c) $90^{\circ}$-ply-Phase-Field, \#3

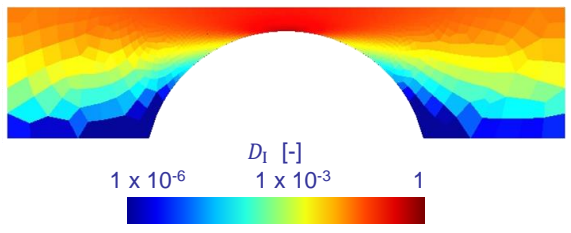

(e) $0^{\circ}$-ply-Phase-Field, \#3

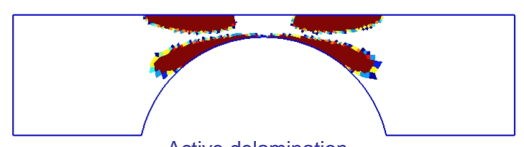

Active delamination

(g) $0^{\circ} / 90^{\circ}$-interface-Phase-Field, \#3

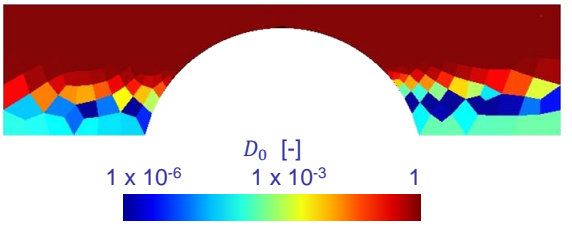

(b) $0^{\circ}$-ply-Phase-Field, \#4

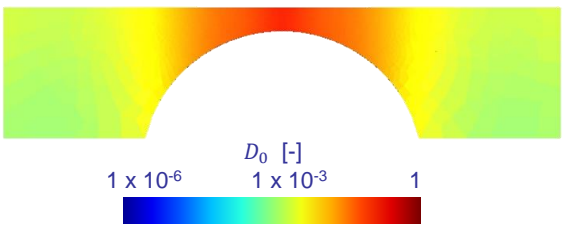

(d) $90^{\circ}$-ply-Phase-field, \#4

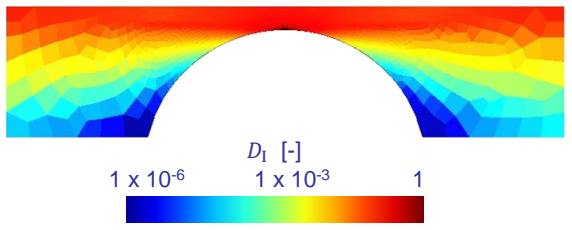

(f) $0^{\circ}$-ply-Phase-field, \#4

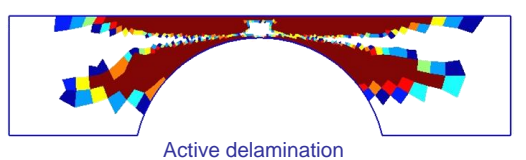

(h) $0^{\circ} / 90^{\circ}$-interface-Phase-Field, \#4

Figure 13: Damage and delamination distributions for the notched sample at configuration \#3 (left column) and at configuration \#4 (right column), see Fig. 10, predicted with the phase-field formulation of the fibre bundle damage process: (a-b) Matrix damage (logarithmic scale) in the $0^{\circ}$ ply; (c-d) Matrix damage (logarithmic scale) in the $90^{\circ}$ ply; (e-f) Fibre bundle damage (logarithmic scale) in the $0^{\circ}$ ply; and (g-h) Delamination zones at the $0^{\circ}-90^{\circ}$ interface. 
figuration $\# 2$, the fibre bundle damage in the $0^{\circ}$-ply has localised with the local approach, see Fig. 12(e), whilst it extends along the fibre orientation with the phase-field method, see Fig. 12(f), The matrix damage distributions are comparable with the experimental observations of Fig. 9(g), with a $0^{\circ}$ splits in the $0^{\circ}$-ply and transverse cracking in the $90^{\circ}$-ply, see Figs. $12(\mathrm{a})+12(\mathrm{~b})$ and Figs. $12(\mathrm{c}), 12(\mathrm{~d})$, respectively. The delamination zone has extended from the notch as seen in Figs. 12(g), 12(h), and is less extended than in the experimental observation of Fig. 9(g). It is actually in better agreement with the CT images of the previous stage, Fig. 9(f).

At this point the local approach looses convergence because of the fibre bundle damage localisation, see Fig. 12(e). The phase-field simulation allows capturing the maximum loading, i.e. configuration \#3 see Fig. 13(left column), and the failed configuration, i.e. configuration \#4 see Fig. 13(right column). Compared to configuration \#2, the $0^{\circ}$ splits first increases in damage amplitude, see Fig. 13(a), and extends to a large region at total failure, see Fig. 13(b). The transverse cracking in the $90^{\circ}$-ply, tends to localise in bands along the fibre directions, see Figs. 13(c) $13(\mathrm{~d})$. The fibre bundle damage in the $0^{\circ}$-ply extends across the cross-section, see Figs. 13(e) 13(f), yielding loss of stress carrying capacity of the laminate. Finally, the delamination zone develops, see Figs. 13(g), 13(h) as already experimentally observed at $80 \%$ of the total load in Fig. 9(h).

\subsection{Applications on a woven unit cell}

In this section we apply the MFH model to represent the yarn behaviour of a plain woven composite material made of the 8552 epoxy resin reinforced with AS4 fibre. The 8552 epoxy properties are used as such for the matrix 


\subsubsection{Geometry}

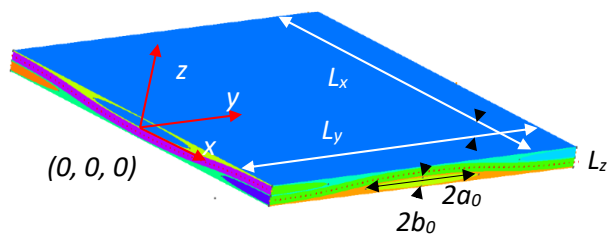

(a) Unit cell

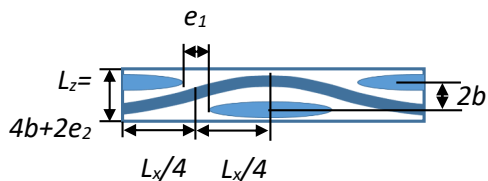

(b) Cross-section

Figure 14: Definition of the plain woven unit cell: dimensions associated to (a) The 3D cell; and to (b) The cross-section.

The geometrical model of the plain woven unit cell represented in Fig. 14 lies on the following assumptions

- The yarns cross-section is approximated by an ellipse of semi-axes $a_{0}$ and $b_{0}$, see Fig. 14(a);

- The size of the unit cell is $L_{x} \times L_{y} \times L_{z}$, see Fig. 14(a)

- The central axis vertical location of a yarn along $\zeta=x$ or $\zeta=y$ reads

$$
z=b\left[\frac{2}{1+\exp \left(-\frac{l}{2}\left(2 \zeta-\frac{L_{\zeta}}{2}\right)\right)}-1\right] \text { for } \zeta \in\left[0 ; \frac{L_{\zeta}}{2}\right],
$$

where $b$ governs the waviness of the yarn and $l$ its asymptotic behaviour such that the yarn reaches the location $\alpha b$ with $\alpha=\frac{z\left(\frac{L_{\zeta}}{2}\right)}{b}$, see Fig. $14(\mathrm{~b})$

- In order to avoid contact between yarns, the condition $b>b_{0}$ is enforced by constraining $b=\xi b_{0}$ with the eccentricity $\xi>1$. 
Table 7: Geometrical description of the woven unit cell.

\begin{tabular}{|c|c|}
\hline Geometrical relationships & Value \\
\hline Cell length $L_{x}=L_{y}=4 a_{0}+2 e_{1}[\mathrm{~mm}]$ & 3.294 \\
\hline Cell thickness $L_{z}=4 b+2 e_{2}[\mathrm{~mm}]$ & 0.2245 \\
\hline Yarn axis location $b=\xi b_{0}[\mathrm{~mm}]$ & 0.053625 \\
\hline Vertical distance between yarns $\alpha=2\left(\frac{1}{1+\exp \left(-\frac{l L_{x}}{4}\right)}-\frac{1}{2}\right)[-]$ & 0.99889 \\
\hline Experimental measurements & Value \\
\hline Yarn cross-section area $A_{0}\left[\mathrm{~mm}^{2}\right]$ & 0.12 \\
\hline Yarn small semi-axis $b_{0}[\mathrm{~mm}]$ & 0.04875 \\
\hline Yarn large semi-axis $a_{0}=\frac{A_{0}}{\pi b_{0}}[\mathrm{~mm}]$ & 0.78353 \\
\hline Yarns horizontal gap $e_{1}[\mathrm{~mm}]$ & 0.08 \\
\hline Model parameters & Value \\
\hline Yarns vertical gap $e_{2}[\mathrm{~mm}]$ & 0.005 \\
\hline Yarn eccentricity $\xi[-]$ & 1.1 \\
\hline Asymptoticy $l L_{x}[-]$ & 30 \\
\hline
\end{tabular}

- The distances between the yarns in the cross-section is governed by $e_{1}$ and $e_{2}$, see Fig. $14(\mathrm{~b})$,

Using the parameters reported in Table 7 allows obtaining a unit cell with $64.3 \%$ volume fraction of yarns.

\subsubsection{Material properties}

The yarns are modelled using the MFH model with damage enhanced matrix and fibre bundle behaviours presented in Section 3. This model is defined using the Euler angles characterising the initial fibre direction. To this end, since each Gauss integration Point (GP), see Fig. 15, belongs to an 


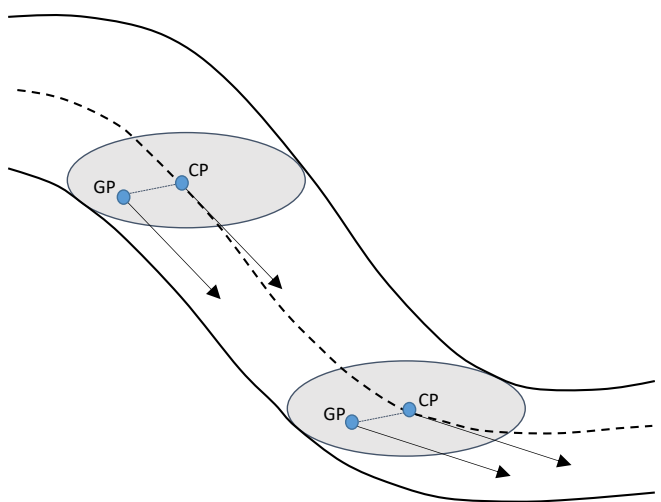

Figure 15: Definition of the non-local MFH model at the Gauss integration Point (GP) from the yarn cross-section defined by its Central Point (CP).

ellipsoidal cross-section, the fibre direction is defined from the normal to the cross-section at its central point, whose directrix is governed by Eq. (129).

The AS4 fibre properties of the yarn are reported in Table 2. The 8552 epoxy properties, using a the saturated damage model, of the yarn are reported in Table 3. These properties are completed by the phase-field model and non-local model parameters of Table 5. Finally we consider that the yarns have a $85 \%$ volume fraction of fibres, yielding a $55 \%$ volume of fibres for the woven unit cell as specified by the manufacturer [58].

The remaining matrix part, i.e. the out-of yarns phase, of the woven unit cell is also modelled with the 8552 epoxy properties reported in Table 3. Since this part has no fibre, the characteristic lengths matrix $\boldsymbol{c}_{0}$ is taken isotropic with the values reported in Table 3 .

Linear tetrahedral elements with volume average volume deformation were used in this simulations. 


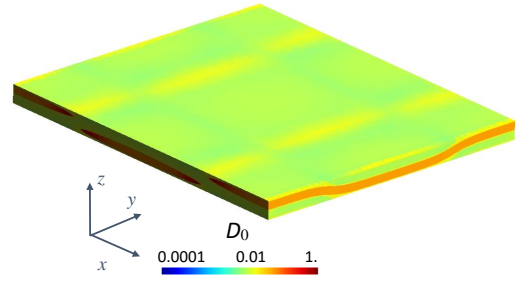

(a) KUBC at $\varepsilon_{x x}=0.017$

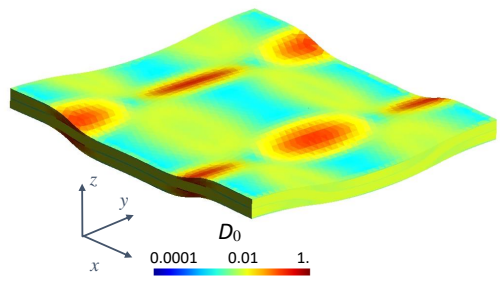

(b) PBC at $\varepsilon_{x x}=0.0145$

Figure 16: Magnified deformation (10 times) and epoxy damage distribution at macrostrain softening onset in the woven unit cell: model with (a) KUBC; and (b) PBC.

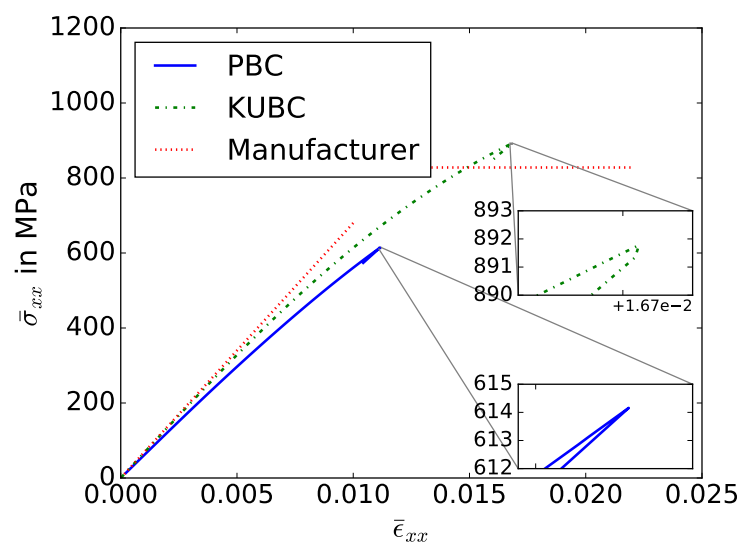

Figure 17: Homogenised stress-strain evolution of the woven unit cell submitted to uniaxial tension; comparison between the results predicted using KUBC and PBC; The manufacturing tensile stiffness and strength are also reported [58].

\subsubsection{Results}

A uni-axial tension is applied on the woven unit-cell. We successively consider the cases in which the lower and upper faces are constrained to i) deform following Periodic Boundary Conditions (PBC) and ii) remain planar following Kinematically Uniform Boundary Conditions (KUBC) in order to study the effect of the out-of-plane deformation mode. For both cases the periodic boundary conditions are considered on the lateral faces although they 
naturally remain planar under uni-axial tension. The resulting (magnified) deformed configurations at macro-strain softening onset are compared in Fig. 16. The PBC model allows out-of-plane deformation and the warp yarns tend to straighten inducing extra deformation in the weft yarns. As a results the predicted homogenised stress-strain curve is more compliant for the PBC model than for the KUBC model, predicting an earlier strain softening onset as illustrated in Fig. 17. The latter figure also reports the manufacturer data [58], which provide only elastic modulus and tensile strength values.

The predictions using the KUBC model are closer to the manufacturing data, both in term of initial slope and strength. This can be explained by the fact that in a real structure the out-of-plane deformations are not totally free because of the laminate-like structure. This behaviour is further studied in Appendix D where it is shown that the response of the layer in laminate unit-cell is closer to that of the KUBC. Besides, as discussed in [60], when comparing the homogenised in-plane Poisson's ratios $\nu_{x y}=0.1$ predicted using $\mathrm{PBC}$, the value is higher than that under $\mathrm{KUBC}\left(\nu_{x y}=\right.$ 0.037). Experimental measurements of in-plane Poisson ratio on a woven composite material are typically $\nu_{x y} \in(0.03,0.05)$ at low strain rate in [61], which is also in better agreement with the KUBC model. Let us note that the analytical result [62] and experimental measurement [63] of the in-plane Poisson ratios for woven fabric have shown $\nu_{x y} \in(0.2,0.57)$. It indicates that the homogenised elasticity properties of woven composites obtained under MBC are more physical than that obtained under PBC.

The damage distributions at damage initiation $\left(\varepsilon_{x x}=0.005\right)$ and at macro-strain softening are illustrated for the different phases in Fig. 18 when 


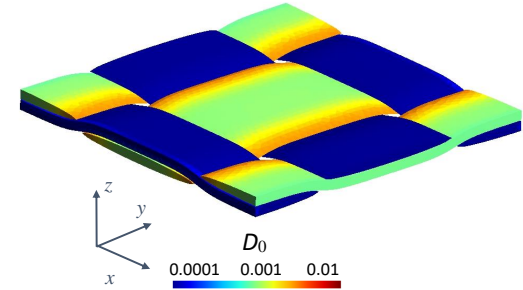

(a) $D_{0}$ in yarns at $\varepsilon_{x x}=0.005$

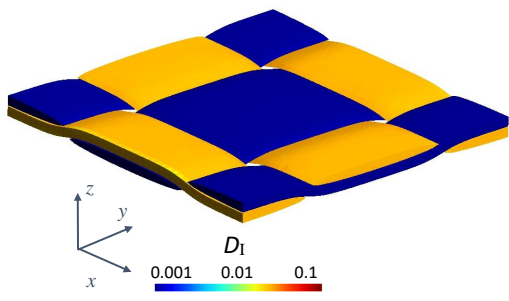

(c) $D_{\text {I }}$ in yarns at $\varepsilon_{x x}=0.005$

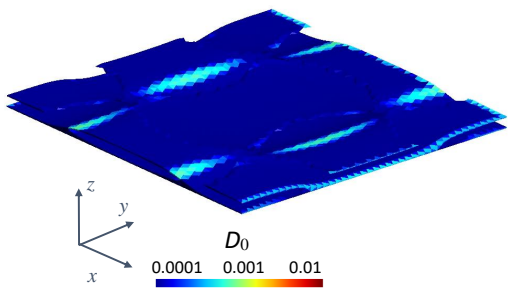

(e) $D_{0}$ in matrix at $\varepsilon_{x x}=0.005$

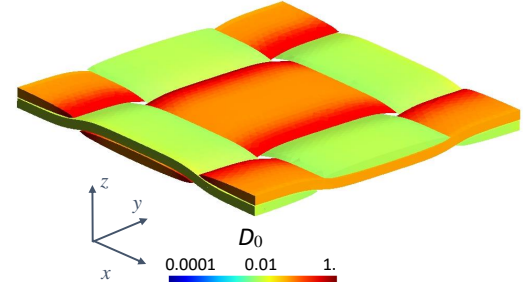

(b) $D_{0}$ in yarns at $\varepsilon_{x x}=0.017$

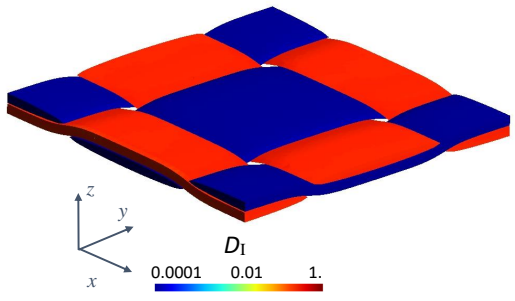

(d) $D_{\text {I }}$ in yarns at $\varepsilon_{x x}=0.017$

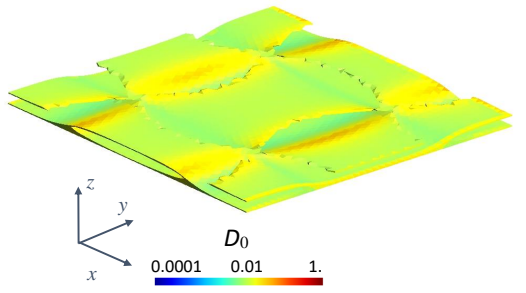

(f) $D_{0}$ in matrix at $\varepsilon_{x x}=0.017$

Figure 18: Evolution of the damage distribution in the woven unit cell simulated using KUBC for a tensile strain $\varepsilon_{x x}=0.005$ (left column) and for a tensile strain $\varepsilon_{x x}=0.017$ (right column): (a-b) Damage $D_{0}$ distribution in the matrix phase of the yarn; (c-d)

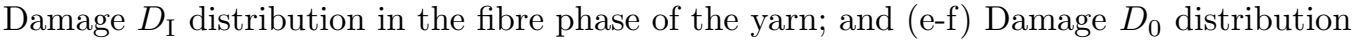
in the matrix (out-of yarns phase). yarns, the damage in the matrix phase propagates in the wefts along a direction parallel to the fibres, i.e. perpendicular to the tensile direction, when 


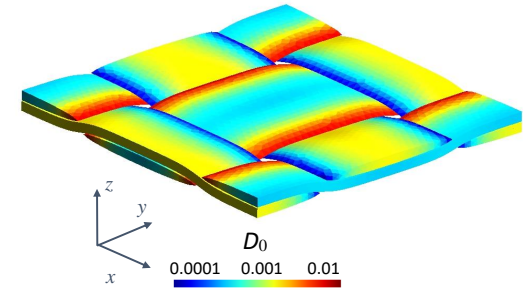

(a) $D_{0}$ in yarns at $\varepsilon_{x x}=0.005$

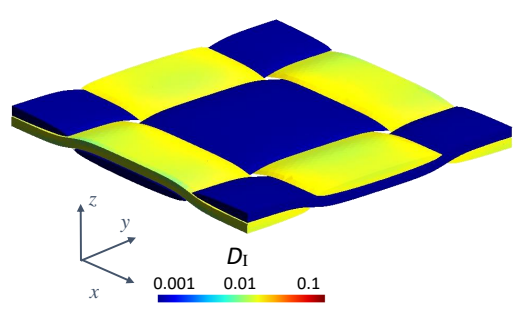

(c) $D_{\text {I }}$ in yarns at $\varepsilon_{x x}=0.005$

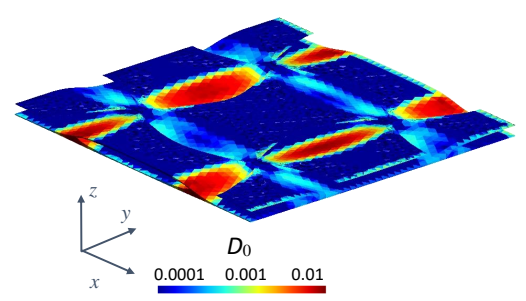

(e) $D$ in matrix at $\varepsilon_{x x}=0.005$

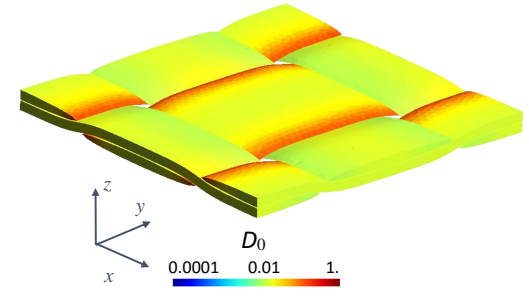

(b) $D_{0}$ in yarns at $\varepsilon_{x x}=0.011$

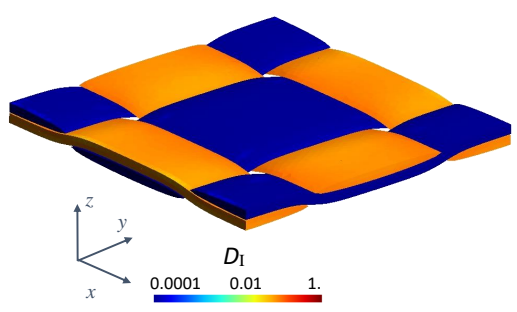

(d) $D_{\text {I }}$ in yarns at $\varepsilon_{x x}=0.011$

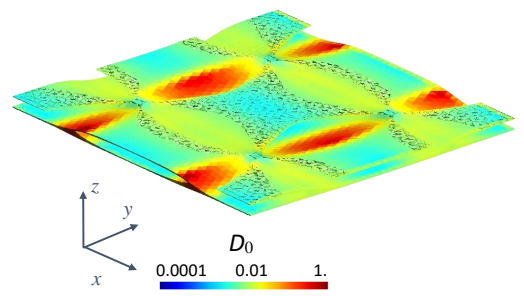

(f) $D$ in matrix at $\varepsilon_{x x}=0.011$

Figure 19: Evolution of the damage distribution in the woven unit cell simulated using PBC for a tensile strain $\varepsilon_{x x}=0.005$ (left column) and for a tensile strain $\varepsilon_{x x}=0.011$ (right column): (a-b) Damage $D_{0}$ distribution in the matrix phase of the yarn; (c-d) Damage $D_{\mathrm{I}}$ distribution in the fibre phase of the yarn; and (e-f) Damage $D$ distribution in the matrix (out-of yarns phase).

considering KUBC, see Figs. 18(a) and 18(b); the final failure is triggered by the fibre damage in the warps, see Figs. 18(d) and 19(d) for respectively the KUBC and PBC cases. Finally it appears that the out-of-yarn epoxy phase 
experiences a damage near the intersections between the warps and wefts, see Figs. 18(f) and 19(f).

\section{Conclusions}

A micro-mechanical model for fibre reinforced matrix has been developed by extending Mean-Field Homogenisation theory to account for fibre bundle breaking and matrix damage. In order to ensure mesh-independence and to recover the correct energy release rate for fibre dominated failure, the damaging process of the fibre bundle has been framed in a phase-field approach. The diffuse damage of the matrix phase has been formulated using an implicit non-local approach. The fibre-matrix interface debonding as well as the matrix yielding and cracking occurring during fibre breaking have been assumed to develop via the evolution of the matrix damage variable [34, which is realistic since the behaviours of the fibre and matrix phases are implicitly coupled.

This micro-structure informed formulation of the UD composite failure presents several features:

- Only micro-structure parameters such as the phase material responses have to be identified to represent the composite UD elastic and elastoplastic responses;

- Knowing the longitudinal critical energy release rate and strength of the fibre-reinforced matrix, which can be obtained by common experimental tests, the phase-field parameters are obtained in order to respect these two values through micro-mechanical argumentation such as the representation of the stress build-up; 
- Correctly representing the energy released during transverse failure can also be done by identification of the non-local characteristic length that allows recovering the transverse critical energy release rate;

- All the required parameters are physical parameters that can be identified easily from either micro-mechanical arguments, manufacturer data sheet, or experimental tests commonly available in the literature, at the exception of the characteristic lengths of the non-local and phasefield models; Although the latter have also a physical meaning, they are identified, on the one hand, in order to recover the transverse critical energy release rate and constrain the matrix cracking direction for the non-local damage model, and, on the other hand, in order to recover the composite material longitudinal strength for the phase-field parameters;

- The anisotropic non-local formulation allowed predicting failure modes such as matrix cracking and fibre failure in good agreement with experimental observation;

- The MFH model is implemented as a classical constitutive material law in a finite element code without particular difficulties, whilst both nonlocal and phase-field formulations require the resolution of additional elliptic equations that have to be integrated at the finite element level, as it is now commonly done finite-element code considering thermomechanical coupling, e.g. or phase-field equations.

In this paper, the material parameters of both fibre and epoxy matrix phases have been identified from manufacturer data sheets in the case of AS4 
fibre reinforced 8552 epoxy matrix. A sensitivity analysis has been conducted on the phase-field model parameters governing the smearing of the damage, whilst constraining the amount of dissipated energy. The model has been studied on the failure of a ply loaded along the longitudinal direction, and it has been shown that the predicted strength is in agreement with the reported values by the manufacturer. The non-local damage parameter of the matrix phase have been identified by micro-mechanical analyses [6, 36].

The developed multi-scale model has first been applied to predict the failure modes of a notched laminate. It was found that the damage delamination patterns were similar to the experimentally observed ones. The multi-scale model has then been applied to represent the yarn failure of a plain woven composite unit-cell under uni-axial tension. To this end, the warps and wefts were modelled as dense unidirectional fibre reinforced epoxy using the developed damage enhanced MFH model.

\section{Appendix A. Material operators of the constitutive models}

\section{Appendix A.1. Damage-enhanced transverse isotropic elasticity}

\section{Appendix A.1.1. Algorithmic operators of damaged fibre bundles}

Because of the existence of the auxiliary damage variable $d_{\mathrm{I}}$, the elastic behaviour of the fibre bundle becomes non-linear, and the stress $\boldsymbol{\sigma}\left(\varepsilon, d_{\mathrm{I}}\right)$ in the fibre bundle depends not only on the fibre strain, but also on the auxiliary damage variable $d_{\mathrm{I}}$. Therefore, the variation of the fibre stress reads

$$
\delta \boldsymbol{\sigma}=\delta\left(\mathbb{C}_{\mathrm{I}}^{\mathrm{D}}: \boldsymbol{\varepsilon}\right)=\mathbb{C}_{\mathrm{I}}^{\mathrm{D}}: \delta \boldsymbol{\varepsilon}+\boldsymbol{\varepsilon}: \frac{\partial \mathbb{C}_{\mathrm{I}}^{\mathrm{D}}}{\partial D_{\mathrm{I}}} \frac{\partial D_{\mathrm{I}}}{\partial d_{\mathrm{I}}} \delta d_{\mathrm{I}}
$$


and the algorithmic operators of the damaged fibres bundle stress read

$$
\begin{aligned}
\mathbb{C}_{\mathrm{I}}^{\varepsilon \varepsilon} & =\frac{\partial \boldsymbol{\sigma}}{\partial \boldsymbol{\varepsilon}}=\mathbb{C}_{\mathrm{I}}^{\mathrm{D}}, \quad \text { and } \\
\boldsymbol{C}_{\mathrm{I}}^{\varepsilon d} & =\frac{\partial \boldsymbol{\sigma}}{\partial d_{\mathrm{I}}}=\varepsilon: \frac{\partial \mathbb{C}_{\mathrm{I}}^{\mathrm{D}}}{\partial D_{\mathrm{I}}} \frac{\partial D_{\mathrm{I}}}{\partial d_{\mathrm{I}}}=n\left(1-d_{\mathrm{I}}\right)^{n-1} \varepsilon: \frac{\partial \mathbb{C}_{\mathrm{I}}^{\mathrm{D}}}{\partial D_{\mathrm{I}}},
\end{aligned}
$$

according to the definition of $D_{\mathrm{I}}$ in Eq. (9).

Besides, in order to solve the coupled system of equations, Eq. (30) also has to be linearised, which requires the evaluation of the following terms

$$
\begin{aligned}
\boldsymbol{C}_{\mathrm{I}}^{\psi \varepsilon}= & \frac{\partial\left(-\frac{l_{\mathrm{I}}}{G_{c}} \frac{\partial \psi_{\mathrm{I}}^{+}}{\partial d_{\mathrm{I}}}\right)}{\partial \varepsilon}=-\frac{n\left(1-d_{\mathrm{I}}\right)^{n-1} l_{\mathrm{I}}}{G_{c}} \frac{\partial \mathbb{C}_{\mathrm{I}}^{\mathrm{D}}}{\partial D_{\mathrm{I}}}: \varepsilon, \text { and } \\
C_{\mathrm{I}}^{\psi d}= & \frac{\partial\left(-\frac{l_{\mathrm{I}}}{G_{c}} \frac{\partial \psi_{\mathrm{I}}^{+}}{\partial d_{\mathrm{I}}}\right)}{\partial d_{\mathrm{I}}}=-\frac{l_{\mathrm{I}}}{2 G_{c}}\left[-n(n-1)\left(1-d_{\mathrm{I}}\right)^{n-2} \varepsilon: \frac{\partial \mathbb{C}_{\mathrm{I}}^{\mathrm{D}}}{\partial D_{\mathrm{I}}}: \boldsymbol{\varepsilon}\right. \\
& \left.+\left[n\left(1-d_{\mathrm{I}}\right)^{n-1}\right]^{2} \varepsilon: \frac{\partial^{2} \mathbb{C}_{\mathrm{I}}^{\mathrm{D}}}{\partial D_{\mathrm{I}}^{2}}: \varepsilon\right]
\end{aligned}
$$

where we have used Eq. 25 and where the derivatives $\frac{\partial \mathbb{C}_{I}^{\mathrm{D}}}{\partial D_{\mathrm{I}}}$ and $\frac{\partial^{2} \mathbb{C}_{\mathrm{I}}^{\mathrm{D}}}{\partial D_{\mathrm{I}}^{2}}$ are respectively given in Appendix A.1.2 and Appendix A.1.3.

Appendix A.1.2. First order derivative of the damaged transverse isotropic elasticity tensor

According to the definition of the damaged transverse isotropic elasticity tensor, Eq. (21), and of $\Delta^{\mathrm{D}}=\left(1+\nu_{\mathrm{I}}^{12}\right)\left(1-\nu_{\mathrm{I}}^{12}-2 \nu_{\mathrm{I}}^{13} \nu_{\mathrm{I}}^{31 \mathrm{D}}\right)$ with $\nu_{\mathrm{I}}^{31 \mathrm{D}}=$ 
${ }_{964}\left(1-D_{\mathrm{I}}\right) \nu_{\mathrm{I}}^{31}$ and $E_{\mathrm{I}}^{3 \mathrm{D}}=(1-D) E_{\mathrm{I}}^{3}$, it yields

$$
\begin{aligned}
\frac{\partial C_{\mathrm{I} 11}^{\mathrm{D}}}{\partial D_{\mathrm{I}}} & =\frac{\partial C_{\mathrm{I} 22}^{\mathrm{D}}}{\partial D_{\mathrm{I}}}=\frac{E_{\mathrm{I}}^{1} \nu_{\mathrm{I}}^{13} \nu_{\mathrm{I}}^{31}}{\Delta^{\mathrm{D}}}-\frac{E_{\mathrm{I}}^{1}\left(1-\nu_{\mathrm{I}}^{13} \nu_{\mathrm{I}}^{31 \mathrm{D}}\right)}{\Delta^{\mathrm{D} 2}} \frac{\partial \Delta^{\mathrm{D}}}{\partial D_{\mathrm{I}}}, \\
\frac{\partial C_{\mathrm{I} 12}^{\mathrm{D}}}{\partial D_{\mathrm{I}}} & =\frac{\partial C_{\mathrm{I} 21}^{\mathrm{D}}}{\partial D_{\mathrm{I}}}=-\frac{E_{\mathrm{I}}^{1} \nu_{\mathrm{I}}^{13} \nu_{\mathrm{I}}^{31}}{\Delta^{\mathrm{D}}}-\frac{E_{\mathrm{I}}^{1}\left(\nu_{\mathrm{I}}^{12}+\nu_{\mathrm{I}}^{13} \nu_{\mathrm{I}}^{31 \mathrm{D}}\right)}{\Delta^{\mathrm{D} 2}} \frac{\partial \Delta^{\mathrm{D}}}{\partial D_{\mathrm{I}}}, \\
\frac{\partial C_{\mathrm{I} 13}^{\mathrm{D}}}{\partial D_{\mathrm{I}}} & =\frac{\partial C_{\mathrm{I}}^{\mathrm{D}} 31}{\partial D_{\mathrm{I}}}=\frac{\partial C_{\mathrm{I} 23}^{\mathrm{D}}}{\partial D_{\mathrm{I}}}=\frac{\partial C_{\mathrm{I} 32}^{\mathrm{D}}}{\partial D_{\mathrm{I}}}, \\
& =-\frac{E_{\mathrm{I}}^{3}\left(\nu_{\mathrm{I}}^{13}+\nu_{\mathrm{I}}^{12} \nu_{\mathrm{I}}^{13}\right)}{\Delta^{\mathrm{D}}}-\frac{E^{3 \mathrm{D}}\left(\nu_{\mathrm{I}}^{13}+\nu_{\mathrm{I}}^{12} \nu_{\mathrm{I}}^{13}\right)}{\Delta^{\mathrm{D} 2}} \frac{\partial \Delta^{\mathrm{D}}}{\partial D_{\mathrm{I}}}, \\
\frac{\partial C_{\mathrm{I} 33}^{\mathrm{D}}}{\partial D_{\mathrm{I}}} & =-\frac{E_{\mathrm{I}}^{3}\left(1-\nu_{\mathrm{I}}^{12} \nu_{\mathrm{I}}^{12}\right)}{\Delta^{\mathrm{D}}}-\frac{E_{\mathrm{I}}^{3 \mathrm{D}}\left(1-\nu_{\mathrm{I}}^{12} \nu_{\mathrm{I}}^{12}\right)}{\Delta^{\mathrm{D} 2}} \frac{\partial \Delta^{\mathrm{D}}}{\partial D_{\mathrm{I}}},
\end{aligned}
$$

with

$$
\frac{\partial \Delta^{\mathrm{D}}}{\partial D_{\mathrm{I}}}=2 \nu_{\mathrm{I}}^{13} \nu_{\mathrm{I}}^{31}\left(1+\nu_{\mathrm{I}}^{12}\right)
$$

$$
\frac{\partial \mathbf{C}_{i j}^{\mathrm{D}}}{\partial d_{\mathrm{I}}}=\frac{\partial \mathbf{C}_{i j}^{\mathrm{D}}}{\partial D_{\mathrm{I}}} \frac{\partial D_{\mathrm{I}}}{\partial d_{\mathrm{I}}} \quad \text { with } \quad i, j=1,2,3 .
$$

Appendix A.1.3. Second order derivative of the damaged transverse isotropic elasticity tensor

Using equation (A.8), the second derivative of $\mathbf{C}^{\mathrm{D}}$ reads

$$
\frac{\partial^{2} \mathbf{C}_{\mathrm{I}}^{\mathrm{D}}}{\partial d_{\mathrm{I}}^{2}}=\frac{\partial^{2} \mathbf{C}_{\mathrm{I}}^{\mathrm{D}}}{\partial D_{\mathrm{I}}^{2}}\left(\frac{\partial D_{\mathrm{I}}}{\partial d_{\mathrm{I}}}\right)^{2}+\frac{\partial \mathbf{C}_{\mathrm{I}}^{\mathrm{D}}}{\partial D_{\mathrm{I}}} \frac{\partial^{2} D_{\mathrm{I}}}{\partial d_{\mathrm{I}}^{2}},
$$


968

with

$$
\begin{aligned}
\frac{\partial^{2} C_{\mathrm{I} 11}^{\mathrm{D}}}{\partial D_{\mathrm{I}}^{2}} & =\frac{\partial^{2} C_{\mathrm{I} 22}^{\mathrm{D}}}{\partial D_{\mathrm{I}}^{2}}=-\frac{2 E_{\mathrm{I}}^{1} \nu_{\mathrm{I}}^{13} \nu_{\mathrm{I}}^{31}}{\Delta^{\mathrm{D} 2}} \frac{\partial \Delta^{\mathrm{D}}}{\partial D_{\mathrm{I}}}+\frac{2 E_{\mathrm{I}}^{1}\left(1-\nu_{\mathrm{I}}^{13} \nu_{\mathrm{I}}^{31 \mathrm{D}}\right)}{\Delta^{\mathrm{D} 3}}\left(\frac{\partial \Delta^{\mathrm{D}}}{\partial D_{\mathrm{I}}}\right)^{2}, \\
\frac{\partial^{2} C_{\mathrm{I} 12}^{\mathrm{D}}}{\partial D_{\mathrm{I}}^{2}} & =\frac{\partial^{2} C_{\mathrm{I} 21}^{\mathrm{D}}}{\partial D_{\mathrm{I}}^{2}}=\frac{2 E_{\mathrm{I}}^{1} \nu_{\mathrm{I}}^{13} \nu_{\mathrm{I}}^{31}}{\Delta^{\mathrm{D} 2}} \frac{\partial \Delta^{\mathrm{D}}}{\partial D_{\mathrm{I}}}+\frac{2 E_{\mathrm{I}}^{1}\left(\nu_{\mathrm{I}}^{12}+\nu_{\mathrm{I}}^{13} \nu_{\mathrm{I}}^{31 \mathrm{D}}\right)}{\Delta^{\mathrm{D} 3}}\left(\frac{\partial \Delta^{\mathrm{D}}}{\partial D_{\mathrm{I}}}\right)^{2}, \\
\frac{\partial^{2} C_{\mathrm{I} 13}^{\mathrm{D}}}{\partial D_{\mathrm{I}}^{2}} & =\frac{\partial^{2} C_{\mathrm{I} 31}^{\mathrm{D}}}{\partial D_{\mathrm{I}}^{2}}=\frac{\partial^{2} C_{\mathrm{I} 23}^{\mathrm{D}}}{\partial D_{\mathrm{I}}^{2}}=\frac{\partial^{2} C_{\mathrm{I}}^{\mathrm{D}} 32}{\partial D_{\mathrm{I}}^{2}}, \\
& =\frac{2 E_{\mathrm{I}}^{3}\left(\nu_{\mathrm{I}}^{13}+\nu_{\mathrm{I}}^{12} \nu_{\mathrm{I}}^{13}\right)}{\Delta^{\mathrm{D}} 2} \frac{\partial \Delta^{\mathrm{D}}}{\partial D_{\mathrm{I}}}+\frac{2 E_{\mathrm{I}}^{3 \mathrm{D}}\left(\nu_{\mathrm{I}}^{13}+\nu_{\mathrm{I}}^{12} \nu_{\mathrm{I}}^{13}\right)}{\Delta^{\mathrm{D} 3}}\left(\frac{\partial \Delta^{\mathrm{D}}}{\partial D_{\mathrm{I}}}\right)^{2}, \\
\frac{\partial^{2} C_{\mathrm{I} 33}^{\mathrm{D}}}{\partial D_{\mathrm{I}}^{2}} & =\frac{2 E_{\mathrm{I}}^{3}\left(1-\nu_{\mathrm{I}}^{12} \nu_{\mathrm{I}}^{12}\right)}{\Delta^{\mathrm{D} 2}} \frac{\partial \Delta^{\mathrm{D}}}{\partial D_{\mathrm{I}}}+\frac{2 E_{\mathrm{I}}^{3 \mathrm{D}}\left(1-\nu_{\mathrm{I}}^{12} \nu_{\mathrm{I}}^{12}\right)}{\Delta^{\mathrm{D} 3}}\left(\frac{\partial \Delta^{\mathrm{D}}}{\partial D_{\mathrm{I}}}\right)^{2} \cdot(\mathrm{A} .10)
\end{aligned}
$$

\section{Appendix A.2. Matrix non-local damage model}

\section{Appendix A.2.1. Radial return mapping of enhanced $J_{2}$ plasticity}

During the occurrence of plastic flow, $f=0$ in Eq. $(32), \dot{p}_{0}$ is positive, and the normality rule yields the plastic strain tensor increment

$$
\dot{\boldsymbol{\varepsilon}}^{\mathrm{pl}}=\dot{p}_{0} \boldsymbol{N}_{0}, \quad \text { with } \boldsymbol{N}_{0}=\frac{\partial f}{\partial \hat{\boldsymbol{\sigma}}}=\frac{3}{2} \frac{\operatorname{dev}(\boldsymbol{\sigma})}{\left(1-D_{0}\right) \hat{\sigma}^{\mathrm{eq}}},
$$

where $\boldsymbol{N}_{0}$ is the normal to the yield surface in the effective stress space, and where the equivalent plastic strain $\dot{p}_{0}=\left[\frac{2}{3} \dot{\varepsilon}^{\mathrm{pl}}: \dot{\varepsilon}^{\mathrm{pl}}\right]^{1 / 2}$. The set of internal variables $\boldsymbol{Z}_{0}$ is thus $\left\{p_{0}, \boldsymbol{\varepsilon}^{\mathrm{pl}}\right\}$.

In order for the incremental-secant operator $\mathbb{C}_{0}^{\mathrm{Sr}}$ in the $\mathrm{MFH}$ scheme to be naturally isotropic, it has been suggested in [27, 43] to consider the normal to the plastic flow from the residual state, i.e. using $\boldsymbol{N}=\frac{3}{2} \frac{\operatorname{dev}\left(\hat{\boldsymbol{\sigma}}-\hat{\boldsymbol{\sigma}}_{n}^{\mathrm{res}}\right)}{\sqrt{\frac{3}{2} \operatorname{dev}\left(\hat{\boldsymbol{\sigma}}-\hat{\boldsymbol{\sigma}}_{n}^{\mathrm{res}}\right): \operatorname{dev}\left(\hat{\boldsymbol{\sigma}}-\hat{\boldsymbol{\sigma}}_{n}^{\mathrm{res}}\right)}}$ as a normal direction in Eq. A.11.

\section{Appendix A.2.2. Algorithmic operators of the matrix damage model}

Because of the existence of the non-local damage variable $\tilde{p}_{0}$, the damageenhanced elasto-plastic response can be stated as $\boldsymbol{\sigma}\left(\varepsilon, \tilde{p}_{0}\right)$, with the lineari- 
sation

$$
\delta \boldsymbol{\sigma}=\mathbb{C}_{0}^{\varepsilon \varepsilon}: \delta \varepsilon+\boldsymbol{C}_{0}^{\varepsilon \tilde{p}} \delta \tilde{p}_{0}
$$

with the material operators of the constitutive law (33) reading

$$
\begin{aligned}
\mathbb{C}_{0}^{\varepsilon \varepsilon} & =\frac{\partial \boldsymbol{\sigma}}{\partial \boldsymbol{\varepsilon}}=\left(1-D_{0}\right) \mathbb{C}_{0}^{\mathrm{alg}}-\hat{\boldsymbol{\sigma}} \otimes \frac{\partial D_{0}}{\partial \boldsymbol{\varepsilon}} \\
\boldsymbol{C}_{0}^{\varepsilon \tilde{p}} & =\frac{\partial \Delta \boldsymbol{\sigma}}{\partial \tilde{p}_{0}}=-\hat{\boldsymbol{\sigma}} \frac{\partial D_{0}}{\partial \tilde{p}_{0}}
\end{aligned}
$$

where $\mathbb{C}_{0}^{\text {alg }}=\frac{\partial \hat{\sigma}}{\partial \varepsilon}$ is the algorithmic operator of the undamaged stress detailed here below. Besides, in order to solve the coupled system of equations, Eq. (38) also has to be linearised, which requires the evaluation of the following terms

$$
\begin{aligned}
\boldsymbol{C}_{0}^{p \varepsilon} & =\frac{\partial p_{0}}{\partial \varepsilon}=\frac{2 \mu_{0}}{h_{0}} \boldsymbol{N}_{\mathbf{0}} \quad \text { and } \\
C_{0}^{p \tilde{p}} & =\frac{\partial p_{0}}{\partial \tilde{p}_{0}}=0,
\end{aligned}
$$

with $h_{0}=3 \mu_{0}+\frac{\partial R_{0}}{\partial p_{0}}$ as detailed here below.

In the case of the radial return mapping assumption, the derivative of the undamaged stress increment with respect to the strain increment reads (e.g. [64, chapter 12])

$\mathbb{C}_{0}^{\mathrm{alg} 0}=\frac{\partial \Delta \hat{\boldsymbol{\sigma}}}{\partial \Delta \boldsymbol{\varepsilon}}=\mathbb{C}_{0}^{\mathrm{el}}-\frac{\left(2 \mu_{0}\right)^{2}}{h_{0}} \boldsymbol{N}_{0} \otimes \boldsymbol{N}_{0}-\frac{\left(2 \mu_{0}\right)^{2}\left(\Delta p_{0}\right)}{\hat{\sigma}^{\mathrm{eq}, \mathrm{tr}}}\left(\frac{3}{2} \mathbb{I}^{\mathrm{dev}}-\boldsymbol{N}_{0} \otimes \boldsymbol{N}_{0}\right)$,

${ }_{993}$ with $\hat{\sigma}^{\mathrm{eq}, \operatorname{tr}}=\sqrt{\frac{3}{2} \operatorname{dev}\left(\hat{\boldsymbol{\sigma}}^{\mathrm{tr}}\right): \operatorname{dev}\left(\hat{\boldsymbol{\sigma}}^{\mathrm{tr}}\right)}$ the equivalent stress of the elastic predictor $\hat{\boldsymbol{\sigma}}^{\mathrm{tr}}=\hat{\boldsymbol{\sigma}}_{n}+\mathbb{C}^{\mathrm{el}}: \Delta \boldsymbol{\varepsilon}$ used in the radial return mapping, $\Delta p_{0}$ the accumulated plastic strain increment, the coefficient $h_{0}=3 \mu_{0}+\frac{\partial R_{0}}{\partial p_{0}}>0$ and the normal direction which reads $\boldsymbol{N}_{0}=\frac{3}{2} \frac{\operatorname{dev}(\hat{\boldsymbol{\sigma}})}{\hat{\sigma}^{\mathrm{eq}}}$, with $\hat{\sigma}^{\mathrm{eq}}=\sqrt{\frac{3}{2} \operatorname{dev}(\hat{\boldsymbol{\sigma}}): \operatorname{dev}(\hat{\boldsymbol{\sigma}})}$. 
When performing the incremental-secant formulation, and in order to define the incremental-secant operator as isotropic in the case in which the residual was not neglected, the radial return mapping was modified to point to the residual stress, with $\boldsymbol{N}=\frac{3}{2} \frac{\operatorname{dev}\left(\hat{\boldsymbol{\sigma}}-\hat{\boldsymbol{\sigma}}_{n}^{\mathrm{res}}\right)}{\left(\hat{\boldsymbol{\sigma}}-\hat{\boldsymbol{\sigma}}_{n}^{\mathrm{res}}\right)^{\mathrm{eq}}}$, where the equivalent effective stress increment reads $\left(\hat{\boldsymbol{\sigma}}-\hat{\boldsymbol{\sigma}}_{n}^{\text {res }}\right)^{\mathrm{eq}}=\sqrt{\frac{3}{2} \operatorname{dev}\left(\hat{\boldsymbol{\sigma}}-\hat{\boldsymbol{\sigma}}_{n}^{\text {res }}\right): \operatorname{dev}\left(\hat{\boldsymbol{\sigma}}-\hat{\boldsymbol{\sigma}}_{n}^{\text {res }}\right)}$. Equation (A.17) thus becomes $\mathbb{C}_{0}^{\mathrm{alg} \mathrm{r}}=\frac{\partial \Delta \hat{\boldsymbol{\sigma}}}{\partial \Delta \boldsymbol{\varepsilon}}=\mathbb{C}_{0}^{\mathrm{el}}-\frac{\left(2 \mu_{0}\right)^{2}}{h} \boldsymbol{N} \otimes \boldsymbol{N}-\frac{\left(2 \mu_{0}\right)^{2}\left(\Delta p_{0}\right)}{\left(\hat{\boldsymbol{\sigma}}^{\mathrm{tr}}-\hat{\boldsymbol{\sigma}}_{n}^{\mathrm{res}}\right)^{\mathrm{eq}}}\left(\frac{3}{2} \mathbb{I}^{\mathrm{dev}}-\boldsymbol{N} \otimes \boldsymbol{N}\right)$,

with $h=3 \mu_{0}+\frac{1}{3} \boldsymbol{N}: \boldsymbol{N}_{0}^{-1} \frac{\partial R_{0}}{\partial p_{0}}>0$. We note that $h$ and $\boldsymbol{N}$ reduces to $h_{0}$ and $\boldsymbol{N}_{0}$ when the residual stress vanishes.

In the following, $\mathbb{C}_{0}^{\text {alg }}$ holds for either Eq. A.17 or A.18.

The material operators of the constitutive law are then obtained, first for the derivatives of the Cauchy stress tensor (A.12), as

$$
\begin{aligned}
\mathbb{C}_{0}^{\varepsilon \varepsilon} & =\frac{\partial \Delta \boldsymbol{\sigma}}{\partial \Delta \boldsymbol{\varepsilon}}=\left(1-D_{0}\right) \mathbb{C}_{0}^{\mathrm{alg}}-\hat{\boldsymbol{\sigma}} \otimes \frac{\partial D_{0}}{\partial \boldsymbol{\varepsilon}}, \\
\boldsymbol{C}_{0}^{\varepsilon \tilde{p}} & =\frac{\partial \Delta \boldsymbol{\sigma}}{\partial \tilde{p}_{0}}=-\hat{\boldsymbol{\sigma}} \frac{\partial D_{0}}{\partial \tilde{p}_{0}} .
\end{aligned}
$$

and then for the derivatives of the equivalent local plastic strain (38) with the operators A.15 A.16 reading

$$
\begin{aligned}
\boldsymbol{C}_{0}^{p \varepsilon} & =\frac{\partial p_{0}}{\partial \Delta \varepsilon}=\frac{2 \mu_{0}}{h_{0}} \boldsymbol{N}_{\mathbf{0}} \quad \text { or } \boldsymbol{C}_{0}^{p \varepsilon}=\frac{2 \mu_{0}}{h} \boldsymbol{N}, \\
C_{0}^{p \tilde{p}} & =\frac{\partial p_{0}}{\partial \tilde{p}_{0}}=0 .
\end{aligned}
$$

These expressions are completed by the linearisation of the damage law (35) written in the incremental form following [42]:

$$
\Delta D_{0}=\left(\frac{\psi_{0_{n+\alpha}}}{S_{0}}\right)^{s_{0}} \Delta \tilde{p}_{0}
$$


1012

where

$$
\psi_{0}=\frac{1}{2} \varepsilon^{\mathrm{e}}: \mathbb{C}_{0}^{\mathrm{el}}: \varepsilon^{\mathrm{e}} \quad \text { and } \quad \psi_{0_{n+\alpha}}=(1-\alpha) \psi_{0_{n}}+\alpha \psi_{0_{n+1}}
$$

1013

It can be easily deduced that

$$
\frac{\partial \psi_{0_{n+\alpha}}}{\partial \varepsilon^{\mathrm{e}}}: \frac{\partial \varepsilon^{\mathrm{e}}}{\partial \boldsymbol{\varepsilon}}: \delta \boldsymbol{\varepsilon}=\alpha \varepsilon^{\mathrm{e}}: \mathbb{C}_{0}^{\mathrm{alg}}: \delta \boldsymbol{\varepsilon}
$$

1014

leading to

$$
\begin{aligned}
\delta D_{0}\left(\varepsilon, \tilde{p}_{0}\right) & \approx \frac{\partial \Delta D}{\partial \psi_{0_{n+\alpha}}} \frac{\partial \psi_{0_{n+\alpha}}}{\partial \varepsilon^{\mathrm{e}}}: \frac{\partial \varepsilon^{\mathrm{e}}}{\partial \boldsymbol{\varepsilon}}: \delta \boldsymbol{\varepsilon}+\frac{\partial \Delta D_{0}}{\partial \tilde{p}_{0}} \delta \tilde{p}_{0} \\
& =\alpha s_{0} \Delta \tilde{p}_{0} \frac{\psi_{0_{n+\alpha}}^{s_{0}-1}}{S_{0}^{s_{0}}} \boldsymbol{\varepsilon}^{\mathrm{e}}: \mathbb{C}_{0}^{\mathrm{alg}}: \delta \boldsymbol{\varepsilon}+\left(\frac{\psi_{0_{n+\alpha}}}{S_{0}}\right)^{s_{0}} \delta \tilde{p}_{0}
\end{aligned}
$$

1015 When considering the damage law (37), during the damage increase $\chi_{0}=$ $1016 \tilde{p}_{0}$ and one has

$$
D_{0}=\frac{D_{\max _{0}}}{1-\frac{1}{1+\exp \left(s_{0} p_{\mathrm{C}_{0}}\right)}}\left(\frac{1}{1+\exp \left(-s_{0}\left(\tilde{p}_{0}-p_{\mathrm{C}_{0}}\right)\right)}-\frac{1}{1+\exp \left(s_{0} p_{\mathrm{C}_{0}}\right)}\right)
$$

1017

whose derivative reads

$\delta D_{0}\left(\boldsymbol{\varepsilon}, \tilde{p}_{0}\right)=\mathbf{0}: \delta \boldsymbol{\varepsilon}+\frac{D_{\max _{0}}}{1-\frac{1}{1+\exp \left(s_{0} p_{\mathrm{C}_{0}}\right)}}\left(\frac{s_{0} \exp \left(-s_{0}\left(\tilde{p}_{0}-p_{\mathrm{C}_{0}}\right)\right)}{\left[1+\exp \left(-s_{0}\left(\tilde{p}_{0}-p_{\mathrm{C}_{0}}\right)\right]^{2}\right.}\right) \delta \tilde{p}_{0}$

1018

1019

\section{Appendix B. Tensors derivatives}

Appendix B.1. Jacobian matrix of MFH resolution

We here recall the expression of $\mathbf{F}(71)$ :

$$
\mathbf{F}=\mathbb{C}_{0}^{\mathrm{SD}}:\left[\langle\Delta \varepsilon\rangle_{\mathrm{I}}^{\mathrm{r}}-\frac{1}{v_{0}} \mathbb{S}^{-1}\left(\mathrm{I}, \mathbb{C}_{0}^{\mathrm{SD}}\right):\left(\langle\Delta \varepsilon\rangle_{\mathrm{I}}^{\mathrm{r}}-\Delta \varepsilon_{\mathrm{M}}^{\mathrm{r}}\right)\right]-\mathbb{C}_{\mathrm{I}}^{\mathrm{SD}}:\langle\Delta \varepsilon\rangle_{\mathrm{I}}^{\mathrm{r}}
$$


The Jacobian matrix (74) reading

$$
\mathbb{J}=\frac{\partial \mathbf{F}}{\langle\Delta \varepsilon\rangle_{\mathrm{I}}^{\mathrm{r}}}-\frac{v_{\mathrm{I}}}{v_{0}} \frac{\partial \mathbf{F}}{\langle\Delta \varepsilon\rangle_{0}^{\mathrm{r}}},
$$

1020 is detailed as

$$
\begin{aligned}
\frac{\partial \mathbf{F}}{\partial\langle\Delta \varepsilon\rangle_{\mathrm{I}}^{\mathrm{r}}=} & \mathbb{C}_{0}^{\mathrm{SD}}:\left[\mathbb{I}-\frac{1}{v_{0}} \mathbb{S}^{-1}\left(\mathrm{I}, \mathbb{C}_{0}^{\mathrm{SD}}\right)\right]-\mathbb{C}_{\mathrm{I}}^{\mathrm{SD}}-\frac{\partial \mathbb{C}_{\mathrm{I}}^{\mathrm{SD}}}{\partial\langle\Delta \varepsilon\rangle_{\mathrm{I}}^{\mathrm{r}}} 3,4:\langle\Delta \varepsilon\rangle_{\mathrm{I}}^{\mathrm{r}} \\
\frac{\partial \mathbf{F}}{\partial\langle\Delta \varepsilon\rangle_{0}^{\mathrm{r}}}= & \frac{\partial \mathbb{C}_{0}^{\mathrm{SD}}}{\partial\langle\Delta \varepsilon\rangle_{0}^{\mathrm{r}}} 3,4:\left[\langle\Delta \varepsilon\rangle_{\mathrm{I}}^{\mathrm{r}}-\frac{1}{v_{0}} \mathbb{S}^{-1}\left(\mathrm{I}, \mathbb{C}_{0}^{\mathrm{SD}}\right):\left(\langle\Delta \varepsilon\rangle_{\mathrm{I}}^{\mathrm{r}}-\Delta \varepsilon_{\mathrm{M}}^{\mathrm{r}}\right)\right]+ \\
& \frac{1}{v_{0}} \mathbb{C}_{0}^{\mathrm{S} \mathrm{D}} \otimes\left(\langle\Delta \varepsilon\rangle_{\mathrm{I}}^{\mathrm{r}}-\Delta \varepsilon_{\mathrm{M}}^{\mathrm{r}}\right)::\left(\mathbb{S}^{-1} \otimes \mathbb{S}^{-1}\right):: \frac{\partial \mathbb{S}}{\partial\langle\Delta \varepsilon\rangle_{0}^{\mathrm{r}}} .
\end{aligned}
$$

1021 Besides, the other required derivatives read

$$
\begin{aligned}
\frac{\partial \mathbf{F}}{\partial \Delta \varepsilon_{\mathrm{M}}^{\mathrm{r}}}= & \frac{1}{v_{0}} \mathbb{C}_{0}^{\mathrm{SD}}: \mathbb{S}^{-1} \\
\frac{\partial \mathbf{F}}{\partial \tilde{p}_{0}}= & \frac{\partial \mathbb{C}_{0}^{\mathrm{S} D}}{\partial \tilde{p}_{0}}:\left[\langle\Delta \varepsilon\rangle_{\mathrm{I}}^{\mathrm{r}}-\frac{1}{v_{0}} \mathbb{S}^{-1}\left(\mathrm{I}, \mathbb{C}_{0}^{\mathrm{SD}}\right):\left(\langle\Delta \varepsilon\rangle_{\mathrm{I}}^{\mathrm{r}}-\Delta \varepsilon_{\mathrm{M}}^{\mathrm{r}}\right)\right]+ \\
& \frac{1}{v_{0}} \mathbb{C}_{0}^{\mathrm{S} \mathrm{D}} \otimes\left(\langle\Delta \varepsilon\rangle_{\mathrm{I}}^{\mathrm{r}}-\Delta \varepsilon_{\mathrm{M}}^{\mathrm{r}}\right)::\left(\mathbb{S}^{-1} \otimes \mathbb{S}^{-1}\right):: \frac{\partial \mathbb{S}}{\partial \tilde{p}_{0}} \\
\frac{\partial \mathbf{F}}{\partial d_{\mathrm{I}}}= & -\frac{\partial \mathbb{C}_{\mathrm{I}}^{\mathrm{SD}}}{\partial d_{\mathrm{I}}}:\langle\Delta \varepsilon\rangle_{\mathrm{I}}^{\mathrm{r}} .
\end{aligned}
$$

Appendix B.2. Derivatives of the secant operators

Appendix B.2.1. Derivatives of the matrix secant operator

The derivatives of the matrix phase damaged incremental-secant operator (64) read 43.

$$
\begin{aligned}
\frac{\partial \mathbb{C}_{0}^{\mathrm{SD}}}{\partial\langle\Delta \varepsilon\rangle_{0}^{\mathrm{r}}} & =\left(1-D_{0}\right) \frac{\partial \mathbb{C}_{0}^{\mathrm{S}}}{\partial\langle\Delta \varepsilon\rangle_{0}^{\mathrm{r}}}-\mathbb{C}_{0}^{\mathrm{S}} \otimes \frac{\partial D_{0}}{\partial\langle\Delta \varepsilon\rangle_{0}^{\mathrm{r}}} \text {, and } \\
\frac{\partial \mathbb{C}_{0}^{\mathrm{SD}}}{\partial \tilde{p}_{0}} & =-\frac{\partial D_{0}}{\partial \tilde{p}_{0}} \mathbb{C}_{0}^{\mathrm{S}} .
\end{aligned}
$$


The derivative of the matrix phase effective incremental-secant operators (62) reads

$$
\frac{\partial \mathbb{C}_{0}^{\mathrm{S}}}{\partial\langle\Delta \varepsilon\rangle_{0}^{\mathrm{r}}}=2 \mathbb{I}^{\mathrm{dev}} \otimes\left[\frac{1}{6 \mu_{0}^{\mathrm{S}}\left(\left(\langle\Delta \varepsilon\rangle_{0}^{\mathrm{r}}\right)^{\mathrm{eq}}\right)^{2}} \Delta \hat{\boldsymbol{\sigma}}_{0}^{\mathrm{r}}: \mathbb{I}^{\mathrm{dev}}: \mathbb{C}_{0}^{\mathrm{alg}}-\frac{2}{3} \mu_{0}^{\mathrm{S}} \frac{\mathbb{I}^{\mathrm{dev}}:\langle\Delta \varepsilon\rangle_{0}^{\mathrm{r}}}{\left(\left(\langle\Delta \varepsilon\rangle_{0}^{\mathrm{r}}\right)^{\mathrm{eq}}\right)^{2}}\right],
$$

1026 with $\left(\langle\Delta \varepsilon\rangle_{0}^{\mathrm{r}}\right)^{\mathrm{eq}}=\sqrt{\frac{2}{3} \operatorname{dev}\left(\langle\Delta \varepsilon\rangle_{0}^{\mathrm{r}}\right): \operatorname{dev}\left(\langle\Delta \varepsilon\rangle_{0}^{\mathrm{r}}\right)}$. In the case in which $\mathbb{C}_{0}^{\mathrm{Sr}}$ is used, $\mathbb{C}_{0}^{\text {alg }}$ is obtained from Eq. A.18, $\mu_{0}^{\mathrm{S}}$ is defined by Eq. (58), and $\Delta \hat{\boldsymbol{\sigma}}_{0}^{\mathrm{r}}=\hat{\boldsymbol{\sigma}}_{0}-\hat{\boldsymbol{\sigma}}_{0}^{\mathrm{res}}$. In the case in which $\mathbb{C}_{0}^{\mathrm{S} 0}$ is used, $\mathbb{C}_{0}^{\mathrm{alg}}$ is obtained from Eq. A.17, $\mu_{0}^{\mathrm{S}}$ is defined by Eq. 61, and $\Delta \hat{\boldsymbol{\sigma}}_{0}^{\mathrm{r}}=\hat{\boldsymbol{\sigma}}_{0}$.

Finally the missing terms $\frac{\partial D_{0}}{\partial\langle\Delta \varepsilon\rangle_{0}^{\mathrm{r}}}$ and $\frac{\partial D_{0}}{\partial \tilde{p}_{0}}$ are developed in Eq. A.26 or Eq. A.28.

Appendix B.2.2. Derivatives of the fibre bundle secant operator

The derivatives of the fibre bundle phase damaged incremental-secant operator 69) read

$$
\begin{aligned}
\frac{\partial \mathbb{C}_{\mathrm{I}}^{\mathrm{S}}}{\partial\langle\Delta \varepsilon\rangle_{\mathrm{I}}^{\mathrm{r}}} & =\frac{\partial \mathbb{C}_{\mathrm{I}}^{\mathrm{D}}}{\partial D_{\mathrm{I}}} \otimes \frac{\partial D_{\mathrm{I}}}{\partial\langle\Delta \varepsilon\rangle_{\mathrm{I}}^{\mathrm{r}}}=0, \text { and } \\
\frac{\partial \mathbb{C}_{\mathrm{I}}^{\mathrm{S}}}{\partial d_{\mathrm{I}}} & =\frac{\partial \mathbb{C}_{\mathrm{I}}^{\mathrm{D}}}{\partial D_{\mathrm{I}}} \frac{\partial D_{\mathrm{I}}}{\partial d_{\mathrm{I}}},
\end{aligned}
$$

where the last term is obtained from Eq. A.8.

Appendix B.2.3. Derivatives of the Eshelby tensor

One has

$$
\begin{aligned}
\frac{\partial \mathbb{S}}{\partial\langle\Delta \varepsilon\rangle_{0}^{\mathrm{r}}=} & \frac{\partial \mathbb{S}}{\partial \nu_{0}} \otimes\left(\frac{\partial \nu_{0}}{\partial \kappa_{0}^{\mathrm{D}}} \frac{\partial \kappa_{0}^{\mathrm{D}}}{\partial\langle\Delta \varepsilon\rangle_{0}^{\mathrm{r}}}+\frac{\partial \nu_{0}}{\partial \mu_{0}^{\mathrm{SD}}} \frac{\partial \mu_{0}^{\mathrm{SD}}}{\partial\langle\Delta \varepsilon\rangle_{0}^{\mathrm{r}}}\right) \\
= & \frac{\partial \mathbb{S}}{\partial \nu_{0}} \otimes\left[\frac{\partial \nu_{0}}{\partial \kappa_{0}^{\mathrm{D}}}\left(-\kappa_{0} \frac{\partial D_{0}}{\partial\langle\Delta \varepsilon\rangle_{0}^{\mathrm{r}}}\right)+\right. \\
& \left.\frac{\partial \nu_{0}}{\partial \mu_{0}^{\mathrm{SD}}}\left(\left(1-D_{0}\right) \frac{\partial \mu_{0}^{\mathrm{S}}}{\partial\langle\Delta \varepsilon\rangle_{0}^{\mathrm{r}}}-\mu_{0}^{\mathrm{S}} \frac{\partial D_{0}}{\partial\langle\Delta \varepsilon\rangle_{0}^{\mathrm{r}}}\right)\right],
\end{aligned}
$$


and similarly

$$
\frac{\partial \mathbb{S}}{\partial \tilde{p}_{0}}=\frac{\partial \mathbb{S}}{\partial \nu_{0}} \otimes\left[\frac{\partial \nu_{0}}{\partial \kappa_{0}^{\mathrm{D}}}\left(-\kappa_{0} \frac{\partial D_{0}}{\partial \tilde{p}_{0}}\right)+\frac{\partial \nu_{0}}{\partial \mu_{0}^{\mathrm{SD}}}\left(-\mu_{0}^{\mathrm{S}} \frac{\partial D_{0}}{\partial \tilde{p}_{0}}\right)\right],
$$

where the derivative $\frac{\partial \mu_{0}^{\mathrm{S}}}{\partial\langle\Delta \varepsilon\rangle_{0}^{\mathrm{r}}}$ is obtained as for Eq. $\mathrm{B.9}$, and $\frac{\partial D_{0}}{\partial\langle\Delta \varepsilon\rangle_{0}^{\mathrm{r}}}$ and the derivatives $\frac{\partial D_{0}}{\partial \tilde{p}_{0}}$ are developed in either Eq. A.26 or Eq. A.28.

\section{Appendix C. Determination of matrix non-local length}

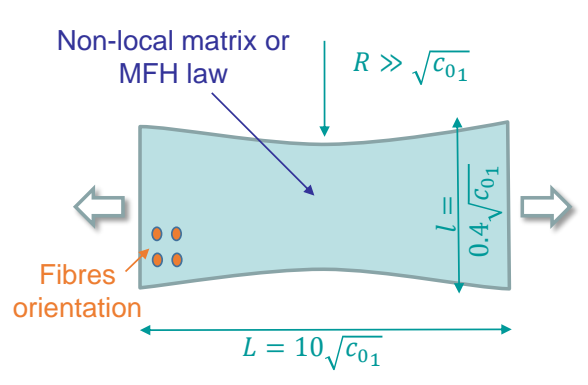

(a) Geometry

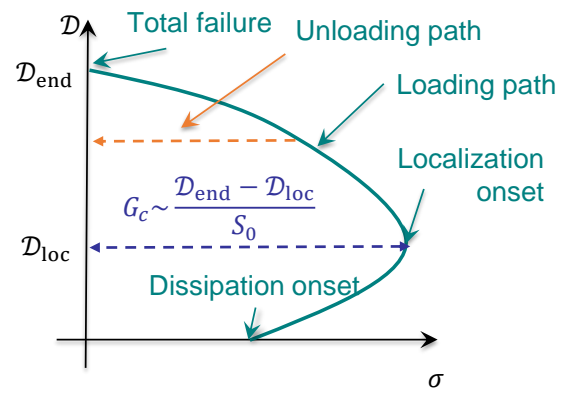

(b) Dissipated energy

Figure C.20: Test performed to evaluate the matrix non-local length from the fracture energy: (a) Geometry of the specimen of length $L=10 \sqrt{c_{0_{1}}}$, width $l=0.2 \sqrt{c_{0_{1}}}$ and of curvature radius $R>>\sqrt{c_{0_{1}}}$; and (b) Failure diagram representing the evolution of the energy dissipation $\mathcal{D}$ with respect to the loading stress $\sigma$. The dissipated energy scales with the volume up to localisation onset $\mathcal{D}_{\text {loc }}$ and then with the cross-section $S_{0}$ (here the width $l$ ).

The critical energy release rate of a material failure process under specific loading conditions, usually denoted by $G_{c}$, measures the total fracture energy released per unit crack surface opening. In our case, as a non-local formalism is adopted, $G_{c}$ is directly related, not only to the damage evolution law chosen, but also to the characteristic lengths $\sqrt{c_{0_{i}}}$ of the non-local matrix 
model. The non-local length can be evaluated by studying a virtual uniaxial traction test in which localisation is triggered by a centred defect as suggested in [36]. The geometry of the virtual specimen is defined by its length $L$, its width $l$ as well as by the curvature radius $R$ which introduces the imperfection, see Fig. 20(a). It has been shown in [36] that the dissipated energy $\mathcal{D}$ scales with the test volume up to localisation onset and, providing $L \gg \sqrt{c_{0_{1}}}$ and $R \gg \sqrt{c_{0_{1}}}$, with the cross-section $S_{0}$, here the width $l$, between the localisation onset and the total failure, see Fig. 20(b). The critical energy release rate $G_{c}$ can be then be estimated from the failure diagram as shown in Fig. 20(b), during the post-peak localisation period, by computing the total energy dissipation and the surface of the cross-section in consideration

$$
G_{c}=\frac{\mathcal{D}_{\mathrm{end}}-\mathcal{D}_{\mathrm{loc}}}{S_{0}},
$$

where $\mathcal{D}_{\text {loc }}$ and $\mathcal{D}_{\text {end }}$ are respectively the accumulated dissipated energies at the onset point of localisation and at the total failure point.

We have performed this virtual test successively on a specimen made of either the bulk epoxy matrix or the UD reinforced epoxy resin. In the latter case, the material law is the damage enhanced MFH scheme with the fibres direction perpendicular to the loading direction. Besides, both the LemaitreChaboche damage law and the saturation damage law described in Section 2.2.2 have been examined for the matrix phase. Figure C.21 illustrates the evolution of the energy release rate $G_{c}$ with respect to the loading stress $\sigma$ on the specimen. It can be seen in Fig. 21(a) that for the failure of the composite material modelled with the damage enhanced MFH scheme, for a given damage law, different values of the non-local length $\sqrt{c_{0_{1}}}$ do not change the peak stress, i.e. the localisation onset, but a longer $\sqrt{c_{0_{1}}}$ leads 


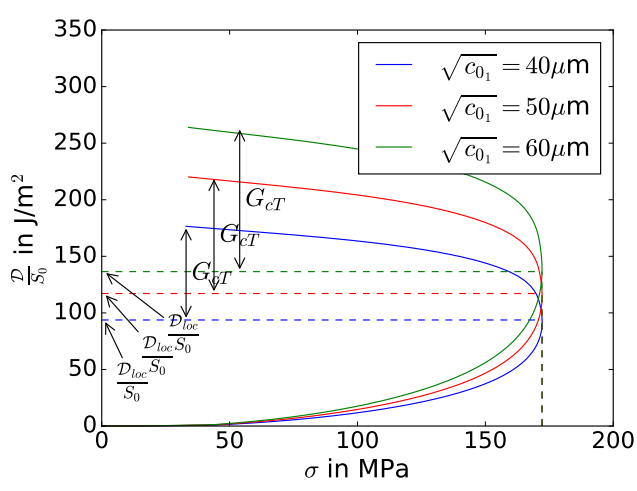

(a) Effect of non-local length

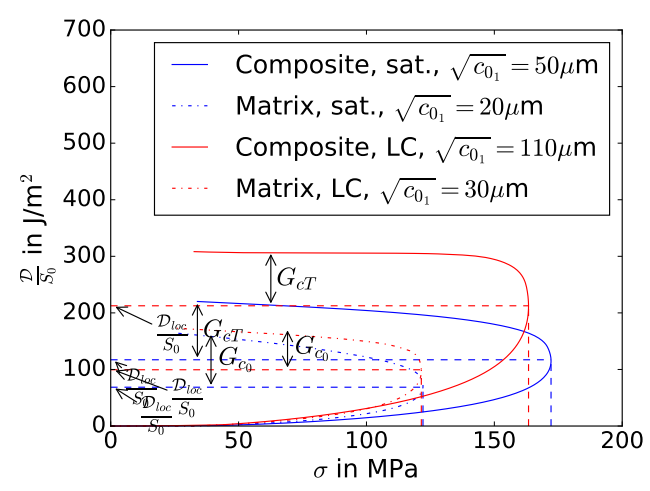

(b) Effect of damage model

Figure C.21: Evaluation of the matrix non-local length in order to recover the fracture energy: (a) Effect of the non-local length $\sqrt{c_{1}}$ on the transverse failure of the AS4 reinforced 8552 epoxy modelled using the saturation damage enhanced MFH; and (b) Recovery of $G_{c T} \simeq 100 \mathrm{~J} / \mathrm{m}^{2}$ for the transverse failure of, on the one hand, the AS4 reinforced 8552 epoxy and of, on the other hand, the bulk matrix; The cases of a Lemaitre-Chaboche model and of a saturation damage law are successively studied.

to a larger $G_{c T}$, which is in agreement with the physical meaning of the non-local characteristic length.

It appears from Fig. 21(a) that to recover the transverse critical energy release rate $G_{c T}$ reported in Table 5 , the non-local length with the saturation law should be selected as $\sqrt{c_{0_{1}}}=50 \mu \mathrm{m}$. Furthermore, repeating the same exercise for the different damage laws and for both the bulk matrix and composite material, Fig. 21(b) allows evaluating the non-local lengths of the bulk matrix as reported in Table 3 and of the non-local matrix model when used in the MFH scheme as reported in Table 5. 


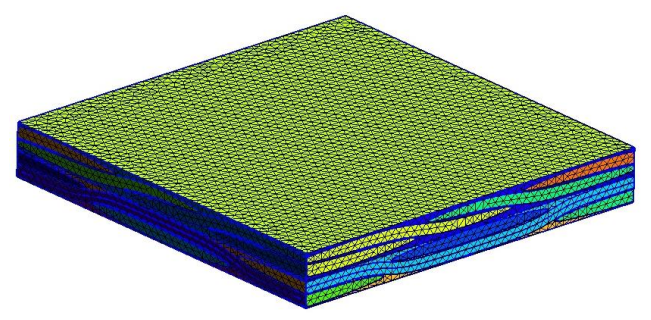

(a) Full mesh

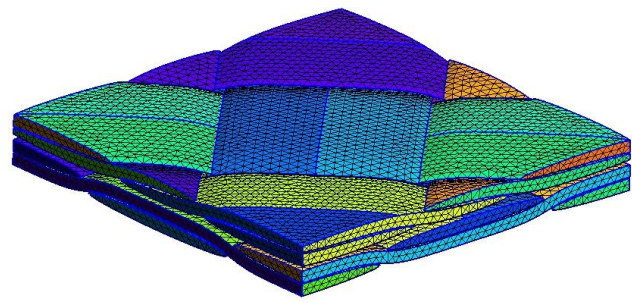

(b) Yarns mesh

Figure D.22: Mesh of a 2-layer $0^{\circ}-90^{\circ} /-45^{\circ}-45^{\circ}$ unit-cell: (a) Full mesh of the unit cell; and (b) Mesh of the yarn.

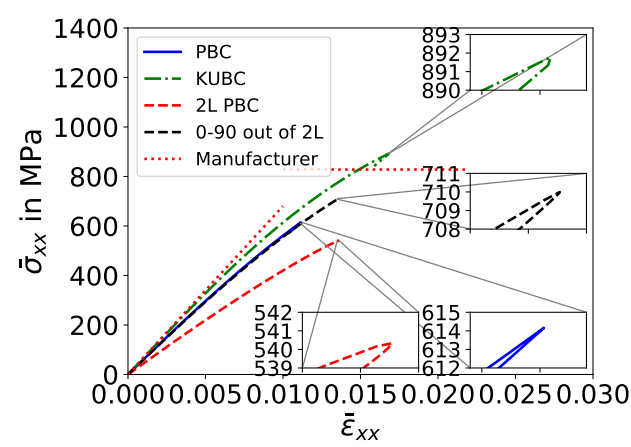

Figure D.23: Comparison of the homogenised stress-strain evolution of the 1-layer $0^{\circ}-90^{\circ}$ unit cell and of the 2-layer $0^{\circ}-90^{\circ} /-45^{\circ}-45^{\circ}$ unit cell submitted to uni-axial tension; The 1-layer $0^{\circ}-90^{\circ}$ unit cell is successively modelled with PBC and KUBC; For the 2-layer $0^{\circ}-90^{\circ} /-45^{\circ}-45^{\circ}$ unit cell the stress-strain response of the full 2-layer unit-cell and of the $0^{\circ}-90^{\circ}$ layer are reported; The manufacturing tensile stiffness and strength are also reported [58].

\section{Appendix D. 2-layer laminate}

In order to assess the representativity of the boundary conditions on the unit cell deformation, we consider the 2-layer $0^{\circ}-90^{\circ} /-45^{\circ}-45^{\circ}$ unit-cell depicted in Fig. D.22 and submit it to PBC. We compare its homogenised stress-strain evolution to the 1-layer unit cell in Fig. D.23. Because of its 
layup, the 2-layer unit-cell is more compliant, so we extracted the response of the $0^{\circ}-90^{\circ}$ layer of the 2-layer unit-cell. It can be seen in Fig. D.23 that the $0^{\circ}-90^{\circ}$ layer of the 2-layer unit-cell exhibits a strength in-between the ones predicted for the 1-layer unit cell with PBC and KUBC. This demonstrates that the real behaviour of the composite layer in a laminate is better represented by the KUBC than by the PBC.

\section{Acknowledgement}

The research has been funded by the Walloon Region under the agreement $\mathrm{n}^{o} .7911-$ VISCOS in the context of the 21st SKYWIN call.

[1] P. van den Heuvel, T. Peijs, R. Young, Failure phenomena in twodimensional multifibre microcomposites: 3. a raman spectroscopic study of the influence of inter-facial debonding on stress concentrations, Composites Science and Technology 58 (1998) 933 - 944.

[2] P. van den Heuvel, S. Goutianos, R. Young, T. Peijs, Failure phenomena in fibre-reinforced composites. part 6: a finite element study of stress concentrations in unidirectional carbon fibre-reinforced epoxy composites, Composites Science and Technology 64 (5) (2004) 645 656. doi:10.1016/j.compscitech.2003.06.003. URL http://www.sciencedirect.com/science/article/pii/ S0266353803003099

[3] A. Scott, M. Mavrogordato, P. Wright, I. Sinclair, S. Spearing, In situ fibre fracture measurement in carbonepoxy laminates using high resolution computed tomography, Composites Science and Technology 71 (12) 
(2011) 1471 - 1477. doi:https://doi.org/10.1016/j.compscitech. 2011.06 .004

[4] A. Arteiro, G. Catalanotti, A. Melro, P. Linde, P. Camanho, Micro-mechanical analysis of the in situ effect in polymer composite laminates, Composite Structures 116 (2014) 827 - 840. doi:https://doi.org/10.1016/j.compstruct.2014.06.014. URL http://wwW.sciencedirect.com/science/article/pii/ S0263822314002839

[5] G. Vigueras, F. Sket, C. Samaniego, L. Wu, L. Noels, D. Tjahjanto, E. Casoni, G. Houzeaux, A. Makradi, J. M. MolinaAldareguia, M. Vzquez, A. Jrusalem, An xfem/czm implementation for massively parallel simulations of composites fracture, Composite Structures 125 (2015) 542 - 557. doi:https: //doi.org/10.1016/j.compstruct.2015.01.053.

URL http://wwW.sciencedirect.com/science/article/pii/ S0263822315001063

[6] L. Wu, D. Tjahjanto, G. Becker, A. Makradi, A. Jérusalem, L. Noels, A micromeso-model of intra-laminar fracture in fiberreinforced composites based on a discontinuous galerkin/cohesive zone method, Engineering Fracture Mechanics 104 (2013) 162 - 183. doi:https://doi.org/10.1016/j.engfracmech.2013.03.018. URL http://www.sciencedirect.com/science/article/pii/ S0013794413001252

[7] J. Guerrero, J. Mayugo, J. Costa, A. Turon, A 3d progressive fail- 
ure model for predicting pseudo-ductility in hybrid unidirectional composite materials under fibre tensile loading, Composites Part A: Applied Science and Manufacturing 107 (2018) 579 - 591. doi:https: //doi.org/10.1016/j.compositesa.2018.02.005.

[8] T. Okabe, H. Sekine, K. Ishii, M. Nishikawa, N. Takeda, Numerical method for failure simulation of unidirectional fiber-reinforced composites with spring element model, Composites Science and Technology 65 (6) (2005) 921 - 933. doi:https://doi.org/10.1016/j. compscitech.2004.10.030.

[9] R. P. Tavares, F. Otero, A. Turon, P. P. Camanho, Effective simulation of the mechanics of longitudinal tensile failure of unidirectional polymer composites, International Journal of Fracture 208 (1) (2017) 269-285.

[10] R. P. Tavares, F. Otero, J. Baiges, A. Turon, P. P. Camanho, A dynamic spring element model for the prediction of longitudinal failure of polymer composites, Computational Materials Science 160 (2019) 42 - 52. doi: https://doi.org/10.1016/j.commatsci.2018.12.048.

[11] F. P. van der Meer, L. J. Sluys, S. R. Hallett, M. R. Wisnom, Computational modeling of complex failure mechanisms in laminates, Journal of Composite Materials 46 (5) (2012) 603-623.

[12] J. Reinoso, G. Catalanotti, A. Blzquez, P. Areias, P. Camanho, F. Pars, A consistent anisotropic damage model for laminated fiber-reinforced composites using the 3d-version of the puck failure criterion, International Journal of Solids and Structures 126-127 (2017) 37 - 53. 
doi:https://doi.org/10.1016/j.ijsolstr.2017.07.023.

URL http://www.sciencedirect.com/science/article/pii/ S0020768317303396

[13] R. P. Tavares, A. R. Melro, M. A. Bessa, A. Turon, W. K. Liu, P. P. Camanho, Mechanics of hybrid polymer composites: analytical and computational study, Computational Mechanics 57 (3) (2016) 405-421.

[14] L. Wu, F. Sket, J. Molina-Aldareguia, A. Makradi, L. Adam, I. Doghri, L. Noels, A study of composite laminates failure using an anisotropic gradient-enhanced damage mean-field homogenization model, Composite Structures 126 (2015) 246 - 264. doi:https://doi.org/10.1016/ j.compstruct.2015.02.070.

[15] F. van der Meer, L. Sluys, Continuum models for the analysis of progressive failure in composite laminates, Journal of Composite Materials 43 (20) (2009) 2131-2156. doi:10.1177/0021998309343054.

[16] C. Miehe, M. Hofacker, F. Welschinger, A phase field model for rateindependent crack propagation: Robust algorithmic implementation based on operator splits, Computer Methods in Applied Mechanics and Engineering 199 (45-48) (2010) 2765-2778. doi:10.1016/j.cma.2010. 04.011 .

[17] T.-T. Nguyen, D. Waldmann, T. Q. Bui, Role of interfacial transition zone in phase field modeling of fracture in layered heterogeneous structures, Journal of Computational Physics 386 (2019) 585-610. doi:https://doi.org/10.1016/j·jcp.2019.02.022. 
URL https://www.sciencedirect.com/science/article/pii/ S0021999119301391

[18] P. Zhang, W. Yao, X. Hu, T. Q. Bui, 3d micromechanical progressive failure simulation for fiber-reinforced composites, Composite Structures 249 (2020) 112534. doi:https: //doi.org/10.1016/j.compstruct.2020.112534. URL https://www.sciencedirect.com/science/article/pii/ S0263822319348421

[19] A. Dean, P. Asur Vijaya Kumar, J. Reinoso, C. Gerendt, M. Paggi, E. Mahdi, R. Rolfes, A multi phase-field fracture model for long fiber reinforced composites based on the puck theory of failure, Composite Structures 251 (2020) 112446. doi:https://doi.org/10.1016/j.compstruct.2020.112446. URL http://wwW.sciencedirect.com/science/article/pii/ S0263822320307078

[20] P. Zhang, X. Hu, T. Q. Bui, W. Yao, Phase field modeling of fracture in fiber reinforced composite laminate, International Journal of Mechanical Sciences 161-162 (2019) 105008. doi:https://doi.org/10.1016/j.ijmecsci.2019.07.007. URL https://www.sciencedirect.com/science/article/pii/ S0020740318341729

[21] P. Zhang, W. Yao, X. Hu, T. Q. Bui, An explicit phase field model for progressive tensile failure of composites, Engineering Fracture Mechanics 241 (2021) 107371. doi:https: 
//doi.org/10.1016/j.engfracmech.2020.107371.

URL https://www.sciencedirect.com/science/article/pii/ S0013794420309516

[22] T. Q. Bui, X. Hu, A review of phase-field models, fundamentals and their applications to composite laminates, Engineering Fracture Mechanics 248 (2021) 107705. doi:https://doi.org/10.1016/j.engfracmech. 2021.107705

[23] R. Hill, Continuum micro-mechanics of elastoplastic polycrystals, Journal of the Mechanics and Physics of Solids 13 (2) (1965) 89 - 101. doi:DOI : $10.1016 / 0022-5096(65) 90023-2$.

[24] D. R. S. Talbot, J. R. Willis, Variational principles for inhomogeneous non-linear media, IMA Journal of Applied Mathematics 35 (1) (1985) 39-54. doi:10.1093/imamat/35.1.39.

[25] H. E. Pettermann, A. F. Plankensteiner, H. J. Böhm, F. G. Rammerstorfer, A thermo-elasto-plastic constitutive law for inhomogeneous materials based on an incremental Mori-Tanaka approach, Computers \& Structures 71 (2) (1999) 197 - 214. doi:DOI:10.1016/S0045-7949(98) $00208-9$

[26] I. Doghri, L. Brassart, L. Adam, J. S. Gérard, A second-moment incremental formulation for the mean-field homogenization of elasto-plastic composites, International Journal of Plasticity 27 (3) (2011) 352 - 371.

[27] L. Wu, L. Noels, L. Adam, I. Doghri, A combined incremental-secant mean-field homogenization scheme with per-phase residual strains for 
elasto-plastic composites, International Journal of Plasticity 51 (2013) 80 - 102. doi:https://doi.org/10.1016/j.ijplas.2013.06.006.

URL http://www.sciencedirect.com/science/article/pii/ S0749641913001174

[28] A. Molinari, G. Canova, S. Ahzi, A self consistent approach of the large deformation polycrystal viscoplasticity, Acta Metallurgica 35 (12) (1987) $2983-2994$.

[29] O. Pierard, I. Doghri, An enhanced affine formulation and the corresponding numerical algorithms for the mean-field homogenization of elasto-viscoplastic composites, International Journal of Plasticity 22 (1) (2006) 131 - 157. doi:10.1016/j.ijplas.2005.04.001.

[30] S. Mercier, A. Molinari, Homogenization of elasticviscoplastic heterogeneous materials: Self-consistent and Mori-Tanaka schemes, International Journal of Plasticity 25 (6) (2009) 1024 - 1048. doi:10.1016/j. ijplas.2008.08.006.

[31] L. Wu, L. Adam, I. Doghri, L. Noels, An incremental-secant mean-field homogenization method with second statistical moments for elastovisco-plastic composite materials, Mechanics of Materials 114 (2017) 180 - 200. doi:https://doi.org/10.1016/j.mechmat.2017.08.006. URL http://www.sciencedirect.com/science/article/pii/ S0167663617300698

[32] R. Peerlings, R. de Borst, W. Brekelmans, S. Ayyapureddi, Gradient- 
enhanced damage for quasi-brittle materials, Int. J. Numer. Meth. Engng 39 (1996) 3391-3403.

[33] I. J. Beyerlein, S. L. Phoenix, Statistics for the strength and size effects of microcomposites with four carbon fibers in epoxy resin, Composites Science and Technology 56 (1) (1996) 75 - 92. doi:https://doi.org/10.1016/0266-3538(95)00131-X. URL http://www.sciencedirect.com/science/article/pii/ $026635389500131 X$

[34] L. Wu, E. Maillard, L. Noels, Tensile failure model of carbon fibre in unidirectionally reinforced epoxy composites with mean-field homogenisation, Composite Structures - (2021) -.

[35] T. Hobbiebrunken, M. Hojo, T. Adachi, C. D. Jong, B. Fiedler, Evaluation of interfacial strength in cf/epoxies using fem and in-situ experiments, Composites Part A: Applied Science and Manufacturing 37 (12) (2006) 2248 - 2256, the 11th USJapan Conference on Composite Materials. doi:https://doi.org/10.1016/j.compositesa.2005.12.021. URL http://wwW.sciencedirect.com/science/article/pii/ S1359835X06000066

[36] V.-D. Nguyen, L. Wu, L. Noels, A micro-mechanical model of reinforced polymer failure with length scale effects and predictive capabilities. validation on carbon fiber reinforced high-crosslinked rtm6 epoxy resin, Mechanics of Materials 133 (2019) 193 - 213. doi:10.1016/j.mechmat. 2019.02.017. 
[37] H. L. Cox, The elasticity and strength of paper and other fibrous materials, British Journal of Applied Physics 3 (3) (1952) 72-79. doi: $10.1088 / 0508-3443 / 3 / 3 / 302$.

[38] R. Peerlings, R. de Borst, W. Brekelmans, M. Geers, Gradient-enhanced damage modelling of concrete fracture, Mech. Cohesive-Frictional Mat. 3 (1998) 323-342.

[39] R. Peerlings, M. Geers, R. de Borst, W. Brekelmans, A critical comparison of nonlocal and gradient-enhanced softening continua, Int. J. Solids Structures 38 (2001) 7723-7746.

[40] J. Lemaitre, Coupled elasto-plasticity and damage constitutive equations, Computer Methods in Applied Mechanics and Engineering 51 (13) (1985) 31 - 49. doi:DOI:10.1016/0045-7825 (85)90026-X.

[41] I. Doghri, Numerical implementation and analysis of a class of metal plasticity models coupled with ductile damage, International Journal for Numerical Methods in Engineering 38 (20) (1995) 3403-3431. doi: $10.1002 / \mathrm{nme} .1620382004$.

[42] J. Lemaitre, R. Desmorat, Engineering damage mechanics: ductile, creep, fatigue and brittle failures, Springer-Verlag, Berlin, 2005.

[43] L. Wu, L. Noels, L. Adam, I. Doghri, An implicit-gradient-enhanced incremental-secant mean-field homogenization scheme for elasto-plastic composites with damage, International Journal of Solids and Structures 50 (24) (2013) $3843-3860$. 
[44] J. D. Eshelby, The determination of the elastic field of an ellipsoidal inclusion, and related problems, Proceedings of the Royal Society of London. Series A, Mathematical and Physical Sciences 241 (1226) (1957) pp. 376-396.

[45] J. Segurado, J. Llorca, A numerical approximation to the elastic properties of sphere-reinforced composites, Journal of the Mechanics and Physics of Solids 50 (10) (2002) 2107 - 2121.

[46] T. Mori, K. Tanaka, Average stress in matrix and average elastic energy of materials with misfitting inclusions, Acta Metallurgica 21 (5) (1973) 571-574, cited By (since 1996) 1814.

[47] D. R. S. Talbot, J. R. Willis, Bounds and self-consistent estimates for the overall properties of nonlinear composites, IMA Journal of Applied Mathematics 39 (3) (1987) 215-240. doi:10.1093/imamat/39.3.215.

[48] P. Ponte Castañeda, The effective mechanical properties of nonlinear isotropic composites, Journal of the Mechanics and Physics of Solids 39 (1) (1991) 45-71. doi:DOI : 10.1016/0022-5096(91)90030-R.

[49] P. Ponte Castañeda, A new variational principle and its application to nonlinear heterogeneous systems, SIAM Journal on Applied Mathematics 52 (5) (1992) 1321-1341.

[50] D. Talbot, J. Willis, Some simple explicit bounds for the overall behaviour of nonlinear composites, International Journal of Solids and Structures 29 (14-15) (1992) 1981 - 1987. doi:10.1016/0020-7683(92) 90188-Y. 
[51] I. Doghri, A. Ouaar, Homogenization of two-phase elasto-plastic composite materials and structures: Study of tangent operators, cyclic plasticity and numerical algorithms, International Journal of Solids and Structures 40 (7) (2003) 1681 - 1712. doi:10.1016/S0020-7683(03) 00013-1.

[52] A. Molinari, F. El Houdaigui, L. Tóth, Validation of the tangent formulation for the solution of the non-linear eshelby inclusion problem, International Journal of Plasticity 20 (2) (2004) 291 - 307. doi: 10.1016/S0749-6419(03)00038-X.

[53] I. Doghri, L. Adam, N. Bilger, Mean-field homogenization of elastoviscoplastic composites based on a general incrementally affine linearization method, International Journal of Plasticity 26 (2) (2010) 219 - 238. doi:10.1016/j.ijplas.2009.06.003.

[54] M. Herráez, A. Fernández, C. S. Lopes, C. González, Strength and toughness of structural fibres for composite material reinforcement, Philos Trans A Math Phys Eng Sci. 374 (2071) (2016) 1-11. doi:https: //doi.org/10.1098/rsta.2015.0274.

[55] S. Pinho, P. Robinson, L. Iannucci, Fracture toughness of the tensile and compressive fibre failure modes in laminated composites, Composites Science and Technology 66 (13) (2006) 2069 - 2079. doi:https://doi.org/10.1016/j.compscitech.2005.12.023. URL http://wwW.sciencedirect.com/science/article/pii/ S026635380600011X 
[56] G. Catalanotti, A. Arteiro, M. Hayati, P. Camanho, Determination of the mode i crack resistance curve of polymer composites using the size-effect law, Engineering Fracture Mechanics 118 (2014) 49-65. doi:https://doi.org/10.1016/j.engfracmech.2013.10.021. URL https://wWW.sciencedirect.com/science/article/pii/ S0013794413003640

[57] Hexcel Corporation, HexPly ${ }^{\complement}$ 8552, Epoxy matrix $\left(180^{\circ} \mathrm{C} / 356^{\circ} \mathrm{F}\right.$ curing matrix), Product Data Sheet (2016).

[58] Hexcel Corporation, HexTow 도 AS4, Carbon Fiber, Product Data Sheet (2018).

[59] F. Naya, C. Gonzlez, C. Lopes, S. Van der Veen, F. Pons, Computational micromechanics of the transverse and shear behavior of unidirectional fiber reinforced polymers including environmental effects, Composites Part A: Applied Science and Manufacturing 92 (2017) 146157. doi:https://doi.org/10.1016/j.compositesa.2016.06.018.

URL https://wWW.sciencedirect.com/science/article/pii/ S1359835X1630197X

[60] L. Wu, L. Adam, L. Noels, Micro-mechanics and data-driven based reduced order models for multi-scale analyses of woven composites, Composite Structures $270 \quad$ (2021) 114058. doi:https://doi.org/10.1016/j.compstruct.2021.114058. URL https://wWW.sciencedirect.com/science/article/pii/ S0263822321005183 


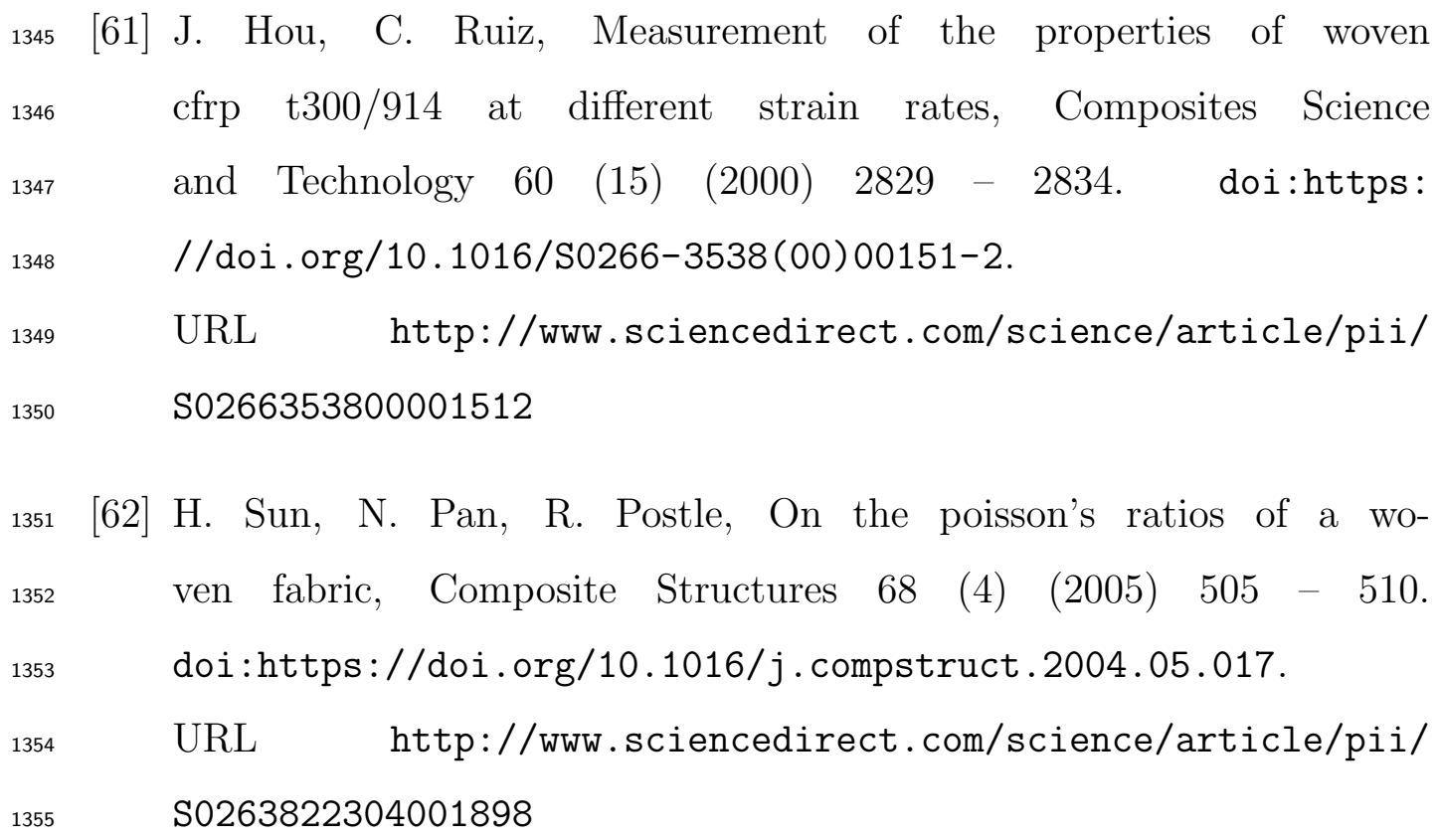

[63] L. Bao, M. Takatera, A. Shinohara, Error evaluation on measuring the apparent poisson's ratios of textile fabrics by uniaxial tensile test, Sen'i Gakkaishi 53 (1) (1997) 20-26. doi:10.2115/fiber.53.20.

[64] I. Doghri, Mechanics of Deformable Solids- Linear, Nonlinear, Analytical and Computational Aspects, Springer-Verlag, Berlin, 2000. 